\title{
Beyond dis-ease and dis-order
}

Citation for published version (APA):

Marsman, A. (2021). Beyond dis-ease and dis-order: exploring the long-lasting impact of childhood adversity in relation to mental health. [Doctoral Thesis, Maastricht University]. Ridderprint. https://doi.org/10.26481/dis.20211103vm

Document status and date:

Published: 01/01/2021

DOI:

10.26481/dis.20211103vm

Document Version:

Publisher's PDF, also known as Version of record

\section{Please check the document version of this publication:}

- A submitted manuscript is the version of the article upon submission and before peer-review. There can be important differences between the submitted version and the official published version of record.

People interested in the research are advised to contact the author for the final version of the publication, or visit the DOI to the publisher's website.

- The final author version and the galley proof are versions of the publication after peer review.

- The final published version features the final layout of the paper including the volume, issue and page numbers.

Link to publication

\footnotetext{
General rights rights.

- You may freely distribute the URL identifying the publication in the public portal. please follow below link for the End User Agreement:

www.umlib.nl/taverne-license

Take down policy

If you believe that this document breaches copyright please contact us at:

repository@maastrichtuniversity.nl

providing details and we will investigate your claim.
}

Copyright and moral rights for the publications made accessible in the public portal are retained by the authors and/or other copyright owners and it is a condition of accessing publications that users recognise and abide by the legal requirements associated with these

- Users may download and print one copy of any publication from the public portal for the purpose of private study or research.

- You may not further distribute the material or use it for any profit-making activity or commercial gain

If the publication is distributed under the terms of Article $25 \mathrm{fa}$ of the Dutch Copyright Act, indicated by the "Taverne" license above, 


\section{BEYOND \\ DIS-EASE \\ \& DIS-ORDER}

EXPLORING THE

LONG-LASTING

IMPACT OF CHILDHOOD

ADVERSITY IN RELATION

TO MENTAL HEALTH

Anve Mousman 



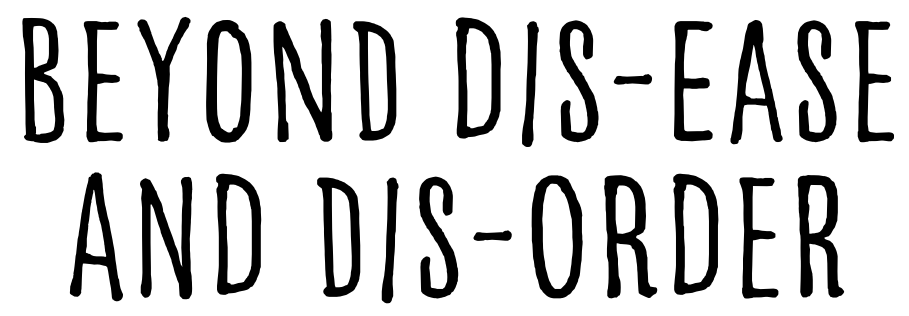

\section{EXPLORING THE LONG-LASTING IMPACT OF CHILDHODD ADVERSITY IN RELATION TO MENTAL HEALTH}

Vera Anne Maria Marsman - Bonekamp 
ISBN: 978-94-6423-347-6

Cover design and layout: (c) evelienjagtman.com

Foto: Gerbrand Langenberg

Printing: Ridderprint | www.ridderprint.nl

Copyright (c) 2021 by Anne Marsman

All rights reserved. No part of this publication may be reproduced, stored in a retrieval system, or transmitted, in any form or by any means, mechanically, electronically, by photocopy, by recording or otherwise, without permission by the author. 


\section{BEYOND DIS-EASE AND DIS-ORDER}

\section{EXPLORING THE LONG-LASTING IMPACT OF CHILDHOOD ADVERSITY IN RELATION TO MENTAL HEALTH}

\section{Proefschrift}

ter verkrijging van de graad van doctor aan de Universiteit Maastricht,

op gezag van de Rector Magnificus,

Prof. dr. Rianne M. Letschert

volgens het besluit van het College van Decanen,

in het openbaar te verdedigen op

woensdag 3 november 2021 om 13.00 uur

Door:

Vera Anne Maria Marsman - Bonekamp

Geboren op 26 december 1989 te Amsterdam 


\section{Promoter}

Prof. dr. Jim van Os

\section{Copromoter}

Dr. Richel Lousberg

\section{Beoordelingscommissie}

Prof. dr. Philippe Delespaul (voorzitter)

Dr. Esther van Duin (Vrije Universiteit Amsterdam)

Prof. dr. Jacqueline Strik

Prof. dr. Wiepke Cahn (Universiteit Utrecht)

The research presented in this dissertation was performed at the School for Mental Health \& Neuroscience, Department of Psychiatry \& Neuropsychology of Maastricht University. 


\section{CONTENTS}

Preface

Chapter 1 General introduction

Chapter 2 Do current measures of polygenic risk for mental disorders contribute to the population variance in mental health?

Chapter 3 Evidence that the impact of childhood trauma on IQ is substantial in controls, moderate in siblings and absent in patients with psychotic disorder

Chapter 4 The impact of adverse childhood experiences on EMG 73 reactivity: a proof-of-concept study

Chapter 5 The influence of childhood adversity on stress sensitivity and 95 habituation to pain

Chapter 6 General discussion

$\begin{array}{lll}\text { Addendum Summary } & 158\end{array}$

Samenvatting $\quad 161$

Impact paragraph 164

$\begin{array}{ll}\text { Curriculum Vitae } & 167\end{array}$

List of publications 169

Dankwoord 171 


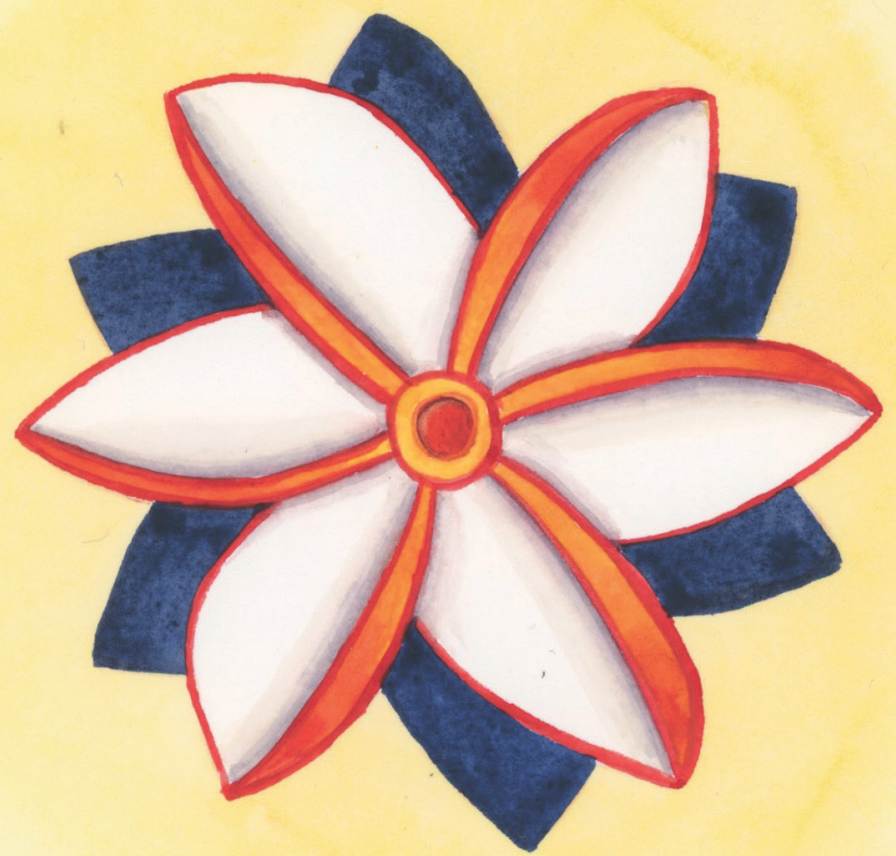


Noem mij, noem mij, spreek mij aan,
o, noem mij bij mijn diepste naam.

Voor wie ik liefheb wil ik heten.

\author{
Neeltje Maria Min
}



PREFACE 


\section{PREFACE}

"It's about shame, disgust, and losing every sense of dignity. About darkness, silence and secrecy. It's about sadness, loneliness, anger and fear. About not being able to express myself or how I feel, and having no boundaries or protection. Basically, it's about not knowing how to just sit, feel and breathe through whatever difficult thought or emotion - without moving to hide it, or fade it, or fix it. It's about looking for safety and comfort in all the wrong directions, and relying on self-destruction in order to live and survive."

These lines belong to the introduction of my bachelor thesis, written in 2014 on what I called a 'human approach' to understanding eating disorders. Based on my own personal experience and the experience of many others, I argued that approaching mental disorders as distinct medical conditions - and treating them as such - can have severe and adverse consequences. Most importantly, because it draws away attention from the fact that above all, mental disorders are part of the human condition: they are human expressions in response to human experiences. Now, several years, a lot of knowledge and experience later, I still argue the same. Passionately.

I was 13 when I first developed what could be called a mental disorder, 17 when I received my first official diagnosis and consequently started treatment, 18 when I became increasingly more skeptical of the DSM and its usefulness, and in my early 20s when I realized that so-called 'evidence-based treatments' and widely accepted theories often did not work for me. In fact, ever since my introduction into the mental health care system as a patient and the start of my psychology study not much later, I mostly felt like they often missed the point. The point being: that the well-defined symptoms of a 'disorder' are not necessarily pathological at all, but first and foremost understandable responses to the circumstances they emerged in. Surely, some symptoms are potentially harmful and clearly deviant of what is to be considered 'normal' or 'healthy' behavior. At the same time, however, they may also help one cope and survive, and thus serve a vital purpose. Self-induced vomiting, for example, while recognized as one of the key pathological features of an eating disorder, can also be considered a powerful method to numb out unbearable feelings and silence agonizing thoughts. Similarly, as I have learned from people with psychosis susceptibility, psychosis may be understood as an ultimate escape from a reality one cannot bear to live in any longer. 
Having said this, I am not implying that mental disorders do not exist, nor that they do not require treatment. They certainly do. And while I am definitely not a fan of biological psychiatry and actively resist the framing of mental disorders as brain disorders, I do appreciate neuroscience and acknowledge the fact that biological factors may play a role in the etiology of mental illness. When it comes to understanding and treating mental illness, however, over the last years, I have increasingly realized the importance of personal diagnostics and the necessity of acknowledging and addressing the context in which symptoms developed. I have also realized that the body in general, and the connection between the body and the mind more specifically, is often ignored and remains significantly unaddressed in widely used theories and treatment methods. While, as I have learned and experienced throughout my life, the body can play such a fundamental role, especially when it comes to understanding and treating the impact of (childhood) adversity.

Based on my own personal experience, and the experience of many others, in the discussion of this dissertation I will argue that perhaps the missing link in the trajectory from childhood adversity to (mental) health problems later in life, is to be found by addressing the body, and all that it embodies. Moreover, by fully acknowledging the complex interplay of biological, psychological and social dimensions that are known to influence all aspects of human life. This, ultimately, may pave the road for us to see and move beyond the legacy of dis-ease and dis-order. 

CHAPTER 1

GENERAL INTRODUCTION 


\section{GENERAL INTRODUCTION}

\section{Introduction}

Mental disorders are anything but uncommon. Worldwide, around 1 billion people suffer from mental illness each year and it is estimated that one in four people will at some point in their lives meet the criteria for any given mental disorder. While mental disorders obviously affect mental health, its impact reaches much further, indirectly also affecting the social and economic well-being of individuals, families, and entire societies as a whole. Hence, mental illness represents an ever-increasing global health problem that requires ongoing awareness and attention.

While mental disorders are typically conceptualized as distinct entities with a set of clearly defined symptoms, in reality there is extensive heterogeneity within and between disorders that challenge the clinical validity and utility of fixed constructs. It is thus suggested, in this dissertation, that symptoms of mental illness are best viewed on a continuum of mental variation and addressed through a process of personal diagnostics, which acknowledges the fact that environmental factors such as exposure to early life stress, may play a significant causal role.

Over the last decades, extensive evidence has emerged that demonstrated the longlasting effects of childhood adversity on later health and well-being. Numerous studies have revealed that adverse childhood experiences (ACE), such as abuse or neglect, can negatively impact neurobiological, immunological, endocrine, and metabolic systems. Further, there is evidence suggesting that exposure to early life stress can influence gene expression and heighten stress sensitivity later in live, increasing the risk of later mental illness.

Although the impact of childhood adversity has received increasing attention over the past decades and many studies have been, and still are, conducted in this field, research gaps remain. Ongoing research examining the impact of childhood adversity from different angles is thus necessary, since it could further help us to understand why some individuals develop mental disorders, while others do not. Consequently, factors that impact vulnerability and resiliency could further be elucidated, eventually leading to advances in prevention and treatment methods.

The work in this dissertation builds on holistic principles and aims to investigate underlying mechanisms that may contribute to the emergence of mental illness, specifically focusing on the trajectory from childhood adversity to mental ill-health later in life. For this purpose, we explore the contribution of both genetic and environmental 
factors in relation to mental health, placing special emphasis on the role of childhood adversity by examining its long-lasting impact on a cognitive, psychological, and psychophysiological level, as well as at the epidemiological level of transition from health to ill-health.

In the following paragraphs of this introduction, I will first briefly address the Dutch 'Schizophrenia does not exist' campaign I have been part of, followed by a short review of the perspective on (ill) health through a biomedical model and biopsychosocial model, after which I will focus on childhood adversity and the long-lasting impact on (mental) health. The introduction will finish with an outline of the different studies in this dissertation and their objectives.

\section{"Schizophrenia does not exist"}

In March 2015, a group of academics, patients, and relatives published an opinion piece in a national newspaper in the Netherlands, stating that we should forget the schizophrenia diagnosis. The authors wrote: "Schizophrenia, mistakenly known as the disease of the 'split mind', does not exist. Psychosis does exist."2 In their article and on the online platform www.psychosenet.nl that was launched the same day, they further explained their rather bold statement, underlining that essentially there is nothing wrong with the name 'schizophrenia' in and of itself. The problem, they argued, is the connotation of a hopeless chronic brain disease and the unscientifically pessimistic view that patients suffering from psychosis are confronted with. ${ }^{3}$

Traditionally, schizophrenia is described as a mental disorder characterized by recurring psychotic episodes that include symptoms such as delusions, hallucinations, disorganized speech, and trouble with thinking. ${ }^{4}$ Within the current fifth edition of the Diagnostic and Statistical Manual of Mental Disorders (DSM-5) schizophrenia is to be found in the category of 'Schizophrenia Spectrum and Other Psychotic Disorders', along with for instance schizoaffective disorder, schizophreniform disorder, delusional disorder and brief psychotic disorder. ${ }^{4}$ Although the DSM conceptualizes mental disorders as distinct entities with a set of clearly defined symptoms, in reality the classification of psychotic disorders (and mental disorders in general) is compromised by substantive heterogeneity within, blurred boundaries between, as well as overlaps across the various disorders in symptom presentation, treatment response and emerging evidence regarding presumed etiology. ${ }^{5,6}$ For this reason, initiators of the 'Schizophrenia does not exist' campaign argued that the different psychosis-related classifications lack clinical validity and are best viewed on a spectrum as part of the same syndrome, namely: psychosis spectrum syndrome - or, as patients have suggested, psychosis susceptibility syndrome. ${ }^{3,7}$ On 
the psychosis spectrum, what is now labeled schizophrenia might represent the minority at the extreme end of this continuum, 'reserved' for those with the most severe and chronic symptomatology.

Indeed, several studies have indicated that only 30\% of all people suffering from psychosis have symptoms that meet the DSM-criteria for schizophrenia. ${ }^{8,9}$ Interestingly though, schizophrenia is at least 10 times more researched than the other $70 \%$ of the psychosis spectrum, leaving other classifications such as schizoaffective disorder and delusional disorder mostly ignored in academic literature and on websites of professional bodies. ${ }^{3}$ Hence, while the concept of schizophrenia only covers a fraction of a much broader spectrum of psychotic disorders, it has basically come to represent everything associated with psychosis - even subtle experiences in a context of depression and/or anxiety ${ }^{10}$ - which, when understood as a distinct, hopeless brain disease, does not exist.

Interestingly, the initiators of the 'Schizophrenia does not exist' campaign in the Netherlands were not the first to propose a different perspective on schizophrenia. Already in 1977, psychologists Joseph Zubin and Bonnie Spring concluded that research on the description and etiology of schizophrenia had come to a standstill and required a new view of the entire subject to move ahead. They offered this view with the publication of their landmark paper 'Vulnerability: A New View of Schizophrenia', in which they explained a new stress-vulnerability model.1" The vulnerability model proposes that each individual is endowed with a degree of vulnerability that under the 'right' circumstances will express itself in an episode of mental illness. In accordance with the dominant view at the time, they stated that this vulnerability may indeed be 'inborn' (i.e., laid down in genes), but - importantly - can also be acquired." Further, they argued that heightened vulnerability to stress, which is thought to be an underlying mechanism in the etiology of psychosis, is not necessarily genetically inherited but can also be acquired via adverse life events and exposure to stressful circumstances.

Unfortunately, while the newly proposed stress-vulnerability model offered a valuable possibility to integrate psychosocial and biological research and catalyze a paradigm shift, the biological approach of schizophrenia as a genetic brain disease remained dominant well into the 21st century.2

\section{The biomedical model}

For centuries, the field of psychiatry has been dominated by a biomedical model that is built on the assumption that each mental illness has a specific biological cause (e.g., pathological changes in neurochemistry or brain structure). ${ }^{13}$ Under the influence of this model, the understanding of mental illness, and human behavioral diversity 
more broadly, became highly medicalized. Medicalization, in this respect, refers to the process of defining previously nonmedical conditions in medical terms, claiming that they are a disease or disease-like entity caused by physiological, biochemical, or genetic abnormalities. ${ }^{14}$ By placing so much emphasis on biological causes, medicalization has proven to be a powerful factor contributing to the objectification of mental disorders. It furthermore gave rise to the dominant force of psychopharmacology in the treatment of mental illness, starting off with the - serendipitous - discovery and use of the first antipsychotic and antidepressant in the 1950s.13 Undoubtedly, the emergence of psychiatric drugs greatly reinforced the framing of mental disorders as biomedical conditions that require disease-specific treatment (e.g., 'anti-psychotic' and 'antidepressant' medication). ${ }^{15}$ It furthermore contributed to the powerful influence of the pharmaceutical industry on how mental health is treated an understood, which we are still confronted with today. ${ }^{16}$

Arguably, schizophrenia is one of the most medicalized mental disorders. For decades, in many academic journals and renowned study books, schizophrenia has been described with terms such as a 'debilitating neurological disorder', a 'devastating, highly heritable brain disorder', and a 'brain disorder with predominantly genetic risk factors'. ${ }^{3}$ Such language is highly suggestive of a distinct, genetic brain disease, in need of a distinct biological treatment. Remarkably, however, to date there is no scientific evidence that fully supports the 'brain disease' claim.'13 To illustrate, a meta-analysis of 80 MRI studies of psychoses published between 1976 and 2015 found no diagnostic or prognostic biomarkers. ${ }^{17}$ Similarly, thousands of studies published on the genetics of schizophrenia have so far failed to identify the 'schizophrenia gene'. Instead, research did consistently demonstrate that most contributing genetic variants are non-specifically associated with a range of mental disorders. ${ }^{18}$ Indeed, it is estimated that approximately two thirds of genetic associations are common to schizophrenia, bipolar disorder, and major depressive disorder ${ }^{6,8,19}$, underlining the notion that there is extensive genetic heterogeneity in human disease. 20

In sum, even after 50 years of neuroscientific research in psychiatry and countless genetic and imaging studies, no biomarkers or otherwise clinically useful results have been found for psychoses, and mental disorders in general. ${ }^{17}$ However, it should be noted that this does not imply that neuroscientific research has been useless. Evidently, it has substantially added to our understanding of brain structures, brain functioning and the nervous system, and this knowledge still is valuable. Yet, we do have to admit that to a great extent genetic and imaging studies lacked - and still lack - clinically useful results that are meaningful to both patients and health care professionals. This may, partially at least, be explained by the fact that most brain research has studied one mental disorder at a time, thereby ignoring existing heterogeneity within and overlap 
between disorders. Perhaps even more importantly, almost all genetic research did not include environmental influences, thereby failing to acknowledge the impact of social, psychological, and other environmental factors that interact with genetic factors in the etiology and maintenance of mental illness. This, in fact, points at one of the biggest shortcomings of the biomedical model: that it leaves no room within its framework for the social, psychological, and behavioral dimensions of (mental) illness.

\section{The biopsychosocial model}

Although the biomedical model for a long time dominated the understanding of and research into mental disorders, growing awareness of the impact of environmental factors resulted in the emergence of several alternative models. One such model worth mentioning is the widely adopted biopsychosocial model. The emergence of this model, conceptualized by cardiologist George Engel in 1977, provided a valuable alternative to the biomedical model by, apart from biological factors, also acknowledging the impact of psychosocial factors in causal and maintaining mechanisms of mental illness. ${ }^{21}$ In line with the earlier mentioned stress-vulnerability model," the biopsychosocial model offers a more holistic approach to (mental) well-being by explaining the complex interplay of three major dimensions, namely: the biological, psychological, and social dimension (figure 1). Each of these dimensions encompass their own specific factors, the social dimension for instance includes factors such education, socioeconomic status and social support, and these factors may interact within and between dimensions.

Importantly, within the biopsychosocial model the possibility of a biological cause or predisposition for (mental) illness is not ignored, nor denied. Engel did however point out that the presence of a biological defect at best defines a necessary but not sufficient condition for the occurrence of an illness. Or, put in his own words: "The abnormality may be present, yet the patient not be ill."21 Remarkably, this insight was actually supported by twin and family studies that examined the genetics and heritability of schizophrenia. Besides confirming that mental illness is to some extent heritable, these twin and family studies more importantly demonstrated that genes alone cannot account for the causation of schizophrenia. ${ }^{19}$ A finding that, already long before the emergence of scientific evidence for the influence of environmental factors, resonated among many health professionals and scientists, of whom George Engel was just one.

Indeed, since the 1960 s researchers have been identifying strong relationships between many environmental factors and mental illness, including for schizophrenia. While in the beginning environmental factors, just like biological factors, were mostly studied in isolation (i.e., a single environmental factor was thought to explain the causation of a specific disorder), it soon became apparent that reality was a lot more complex. Based 
on a great number of epidemiological and psychosocial studies, several general principles about the role of the environment emerged that are worth mentioning. First, the same type of environmental exposure increases the risk of not one, but many different disorders. ${ }^{19}$ For instance, while urban environment was first identified as a specific risk factor for schizophrenia, further research showed that urbanicity is associated with increased risk of all types of mental disorders. ${ }^{22}$ Second, a variety of environmental exposures contribute to risk of the same disorder. Maternal malnutrition, viral infections during pregnancy, loweconomic status, and childhood adversity, for example, are all known to increase the risk of conditions diagnosed as schizophrenia. ${ }^{19}$ Last, and importantly, no fixed constellation of environmental exposures will result in mental illness among all exposed individuals. Indeed, many individuals who are exposed to multiple adverse factors do not develop any mental disorder as a consequence. .3,24 $^{23}$

Thus, it can be concluded that while some factors may put an individual at risk for developing a whole range of problems, other factors (e.g., social support) may be considered protective and enhance resiliency.

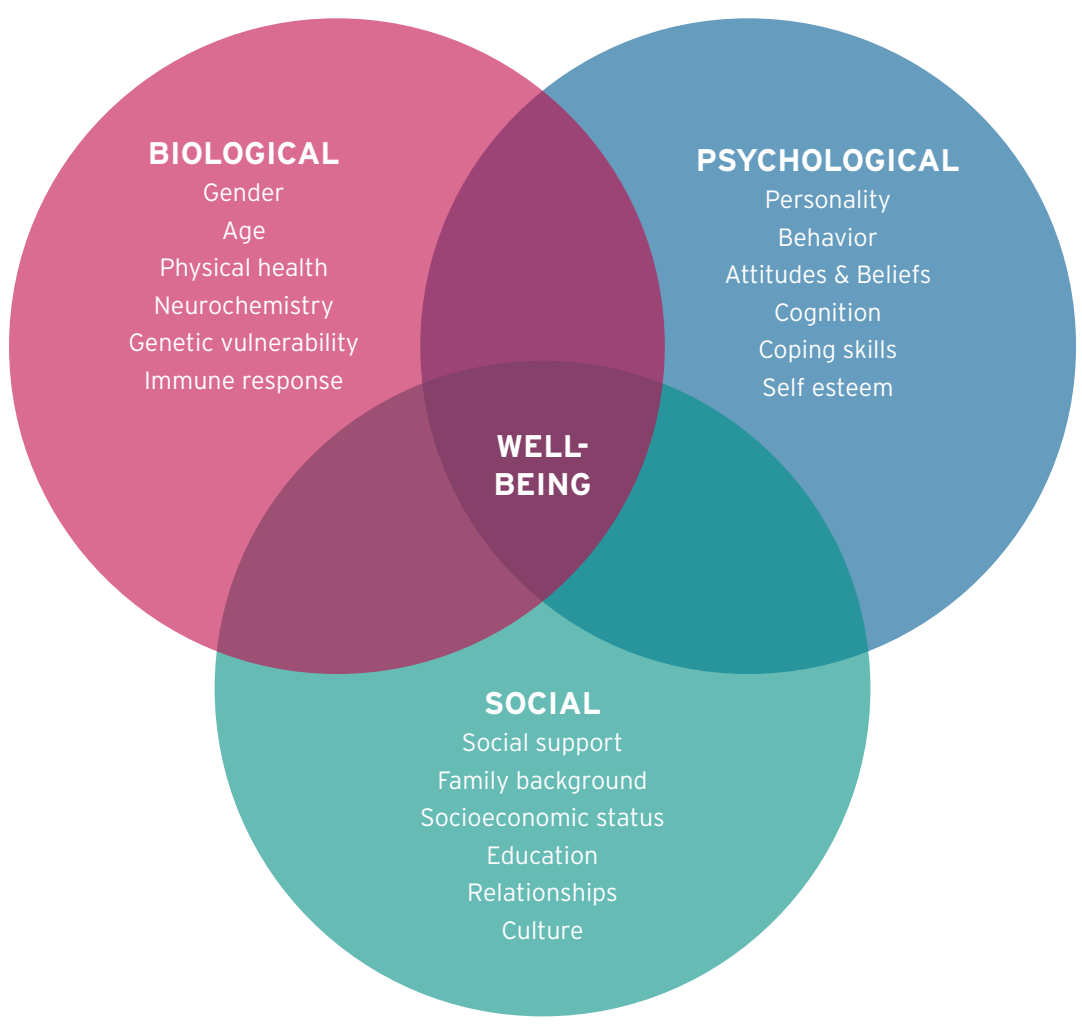

Figure 1. Illustration of the biopsychosocial model 
To summarize, while within the biomedical model mental illness is thought to be primarily caused by a biological defect, the biopsychosocial model recognizes that most likely, biological, and environmental factors jointly and interactively contribute to the causation of mental illness. It thus acknowledges that, just like no genetic variant can independently account for the causation of mental illness, no environmental factor can on its own be a sufficient cause. Over the last few decades, however, it is becoming increasingly clear that certain environmental factors may play a crucial role in the emergence of a wide range of health problems, mental disorders included. In this respect, exposure to stressful circumstances early in life is one such factor that has received growing interest. And, as will be argued in this dissertation, it is perhaps one of the most important factors to reckon with when it comes to understanding the emergence of any given form of dis-ease or dis-order.

\section{The long-lasting impact of childhood adversity}

Since the early writings on mental disorders, many advances have been made in the field of psychiatry that improved the understanding and treatment of mental illness. With respect to unraveling the relationship between childhood adversity and later health and well-being, the Adverse Childhood Experiences (ACE) study ${ }^{25}$ conducted in the Unites States in the early 1990s - played a significant role. In fact. the ACE study still is one of the largest investigations ever undertaken to examine the long-lasting impact of childhood adversity. The examination of more than 17,000 participants in this study yielded numerous valuable and important results, at the time by many experienced as profoundly shocking. In short, the ACE study revealed that ten commonly reported 'adverse childhood experiences' (referred to as ACES) predict a wide range of psychological, medical, and functional problems; that these ACEs often do not exist in isolation but are likely to co-occur; and finally, that a doseresponse relationship exists, implying that more ACEs result in a greater likelihood of developing health problems. ${ }^{25}$

Since the ACE study in the late 1990s, findings have been replicated internationally and research on the impact of stress and trauma has greatly expanded. This has led to many important insights, including the now widely acknowledged fact that there are many other forms of childhood adversity than the ten ACEs that were assessed in the original study (i.e., forms of neglect, abuse, and family dysfunction). Within the existing literature, these potentially harmful experiences are referred to by many terms, such as: early life adversity, early life stress, early life trauma, childhood trauma, adverse childhood experiences and childhood adversity. They typically include - but are not limited to - any form of child abuse, neglect, and maltreatment. ${ }^{26}$ Additional adverse experiences are for instance exposure to natural disasters, bullying, community violence and living in 
poverty. ${ }^{27}$ While it should be acknowledged that the experience and impact of childhood adversity can be highly variable, for instance with respect to the nature of the adverse event(s), its timing and frequency, there is also great commonality to be recognized that may explain part of the underlying mechanism by which early adversity can cause long-lasting impairments. Namely: the fact that adverse childhood experiences per definition are stressful and overwhelming to the child exposed. ${ }^{27,28}$ Accordingly, it is suggested that these two 'features' of childhood adversity play a pivotal role in the etiology of (mental) illness.

While stress is certainly not always bad and can serve important functions, exposure to excessively high and chronic levels of stress can have detrimental effects on both physical and mental health, especially during childhood when the brain and nervous system are still developing..$^{29}$ Indeed, a history of stressful or traumatic experiences in childhood has long been recognized as a significant risk factor for developing numerous mental disorders later in life, including psychotic disorders, mood disorders and eating disorders. ${ }^{29-31}$ Although the 'outcome' seems clear, questions remain concerning why and how childhood adversity impacts these health outcomes.

Extensive human and animal research has provided insights into neurobiological and molecular mechanisms by which early stress may become biologically embedded and subsequently cause impairments. ${ }^{32}$ One of the mechanisms proposed concerns the induction of so called 'toxic stress' and alteration of the normal stress response. Exposure to stress, regardless of its intensity, per definition causes physiological changes such as increased heart rate and cortisol hormonal levels in the body. ${ }^{33}$ Research has shown that stress can be classified into 'good/positive stress', 'bearable stress' and 'toxic stress, ${ }^{29}$ and can have acute, delayed and long-term effects on the brain and the rest of the body. ${ }^{34}$ 'Good' and 'bearable stress' can be very effective, since it may increase focus and endurance and enable someone to fight or flight when confronted with a (negative) stressor. When stress becomes too overwhelming, however, in an acute state it can cause the entire nervous system to shut down and trigger a so called 'freezeresponse'. On the long-term, 'toxic stress' is characterized by prolonged or frequent activation and dysregulation of the normal stress response, inducing biological changes and potential damage to the entire organism. ${ }^{29,34}$

The hypothalamic-pituitary-adrenocortical (HPA)-axis is known to play a fundamental role in the stress response, releasing cortisol when activated. ${ }^{35}$ Besides HPA-axis activation, stress is also known to influence several other brain areas including the prefrontal cortex and limbic brain regions. ${ }^{29}$ These regions are sometimes also termed the 'thinking brain' and the 'emotional brain', as a reference to their main functions. 
Based on a great body of research, it has been suggested that exposure to excessively high and chronic levels of stress (i.e., toxic stress) may dysregulate the normal stress response, resulting in hyperactivity of the HPA-axis, heightened sensitivity for and increased reactivity to stress, which, in turn, is thought to cause increased levels of psychopathology and higher rates of mental disorders.

Hence, toxic stress may indeed get under the skin and alter biological processes, including that of neurobiological, immunological, endocrine, and metabolic systems. ${ }^{28,32}$ The upcoming science of epigenetics - in short, the study of mechanisms that can change gene expression - may further elicit etiological mechanisms. Epigenetic studies suggest that the effect of environmental factors (e.g., childhood adversity) is likely conditional on genetic factors, resulting in gene-environment interactions. Furthermore, it is suggested that impact of environmental factors (e.g., daily life stress) also depends on previous exposures, resulting in environment-environment interactions. Taken together, while epigenetics is still in its infancy, it is becoming increasingly clear that multi-factorial gene-environment interactions are likely to represent a generic mechanism involved in the majority of cases of mental illness. ${ }^{19}$

To conclude, over the last decades, evidence for the lifelong impact of childhood adversity on a biological, psychological, and social dimension has been well established. While questions and research gaps remain concerning underlying mechanisms and factors that impact one's vulnerability or resiliency with respect to mental illness, it is unquestionable that early exposure to stressful or traumatic events can play a crucial role. Hence, with respect to understanding, studying, diagnosing, and treating mental illness, childhood adversity should always be taken into account as a potential risk factor that plays a role in both the causation and maintenance of symptoms, moreover, acknowledging that current pathology may be a result of previous exposure to adversity.

Finally, and perhaps most importantly, it should also be recognized that what is sometimes considered to be a disorder, may in fact not be a problem, but a solution to a problem, or dis-order, and should thus be regarded as such. Recognition of the fact that symptoms can serve an important purpose is thought to be essential, since it can have profound implications for addressing and treating mental illness. These implications may be best illustrated by a profound insight of Vincent Felitti, one of the main researchers of the ACE Study, who stated: "if you mistake someone's solution for a problem to be eliminated, not only are they likely to fail treatment, as often happens in addiction programs, but other problems may emerge".28 


\section{Aims and outline of this dissertation}

Knowledge about the long-lasting impact of childhood adversity on all dimensions related to well-being and health, has the potential to greatly improve the current understanding, prevention, and treatment of mental illness. Although a lot of valuable knowledge is already available, research gaps still exist and etiological underlying mechanisms with respect to mental illness remain to be elucidated. Many studies have already demonstrated the impact of childhood adversity at the (neuro)biological and psychological level, however, relatively little is known about the long-lasting impact on a cognitive and (psycho)physiological level. Since impairments on a cognitive and/or physiological level can greatly impact overall functioning and well-being, enhancing the understanding of how childhood adversity may impact these domains is considered to be important, hence, one of the aims of this dissertation.

While the respective roles of genetic factors and early life stress to transdiagnostic psychopathology are well established, as predicted by the biopsychosocial model, and each is considered important in terms of contribution to risk in twin studies, little is known on how these factors contribute to variance in mental health, and to what extent. In this dissertation, we set out to study this issue in a large populationbased cohort, using modern molecular measures of genetic risk (i.e., polygenic risk scores) and measures of familial and environmental risk on the level of and change in general mental health, in the hope of extending biopsychosocial theory of the ontogenesis of mental ill-health. Conform previous research on the long-lasting impact of childhood adversity, we predict that the contribution of (measurable) early adversity to later mental health is very extensive in comparison to molecular measures of genetic risk.

In the past few years, there has been growing awareness for the fact that trauma (and adversity more broadly) is not just an event that took place in the past, but moreover the imprint that is left by that event on the mind, brain, and body. The scientific bestseller 'The body keeps the score'28 by researcher and psychiatrist Bessel van der Kolk, is possibly one of the most profound and influential books one may (and should) read on this topic. Based on recent scientific advances and the stories of many people who have experienced (childhood) adversity themselves, in this book it is carefully demonstrated that indeed, the body keeps the score, which as a result, may compromise one's capacity for pleasure, engagement, self-regulation, and trust. With respect to the relationship between childhood adversity and the body, previous research has shown that physiological parameters such as heart rate variability and brain activity are interesting markers to examine in an attempt to objectify the impact of stress and trauma. Another interesting 
parameter that is far less examined, yet obviously impacted by stress and furthermore easily measurable, is muscle tension. Indeed, some research exists showing that for instance trapezius muscle stress reactivity may be an interesting candidate to examine the impact of early life stress on a physiological stress-related outcome. ${ }^{36,37}$ Furthermore, there is evidence that exposure to early life stress may cause heightened sensitivity to stress that persists throughout life, ${ }^{38,39}$ which, arguably, may also be measurable on a physiological level (e.g., muscle tension).

In this dissertation, we further explore the hypothesis of increased stress sensitivity, representing an acquired vulnerability that can be traced back to early stress exposure, by investigating the impact of adverse childhood experiences on trapezius muscle reactivity. More specifically, this is done by conducting two different stress experiments, namely, a memory task using a cognitive stressor, and a habituation task using a series of electrical painful stimuli, while measuring electromyography (EMG) stress reactivity. Additionally, since there is a substantial body of literature showing that childhood adversity is a risk factor for developing various types of chronic pain conditions, ${ }^{40,41}$ we also investigate the influence of childhood adversity on habituation to pain.

Another important parameter of bodily functioning is, arguably, cognition. With respect to the relationship between childhood adversity and cognitive functioning, there is growing research demonstrating the detrimental impact of exposure to early life stress on cognitive functioning throughout later life. ${ }^{42,43}$ However, findings have been inconsistent across different cognitive domains, demographic, diagnostic and genotype-subgroups, often based on studies with a relatively small sample size.44,45 In this dissertation, we study the link between childhood adversity and cognition in a longitudinal study, including patients with psychotic disorder, siblings of patients and healthy comparison subjects, taking into account several known issues such as adequate sample size and repeated measures of the cognitive outcome.

Taken together, this dissertation aims to investigate underlying mechanisms that may contribute to the emergence of mental illness, specifically focusing on the trajectory from childhood adversity to mental ill-health later in life. For this purpose, we explore the contribution of both genetic and environmental factors in relation to mental health, placing special emphasis on the role of childhood adversity by examining its long-lasting impact at the cognitive, psychological, and psychophysiological level, as well as at the epidemiological level of transition from health to ill-health. Or, stated differently, from order to dis-order. This dissertation consists of four studies, each addressing the issue from a different perspective. 
Chapter $\mathbf{2}$ explores the contribution of molecular genetic, familial, and environmental risk factors to the variance in level of and change in mental health in a large populationbased cohort. The cohort was examined 4 times over a period of 9 years.

Chapter $\mathbf{3}$ focusses on the link between childhood trauma and cognition. It examines whether cognitive alterations observed in patients with psychotic disorder and their relatives are related to trauma, taking into account adequate sample size, the inclusion of endophenotypic measures of cognition in siblings, and repeated measures of cognitive outcome over time. The sample was examined 3 times over a period of 6 years.

Chapter $\mathbf{4}$ explores the link between childhood adversity and stress-related trapezius muscle activity in a novel stress experiment, using a cognitive stressor in the form of a memory task. The study used a sample of healthy participants and included 2 identical experimental sessions.

Chapter $\mathbf{5}$ builds upon the previous chapter, this time using a physical stressor in the form of a series of painful stimuli. Besides examining the association between childhood adversity and (increased) stress sensitivity, it also examines the impact of childhood adversity on habituation to pain on an objective level (EMG) as well as a subjective level (pain report on a numeric rating scale).

Chapter $\mathbf{6}$ provides a summary and discussion of the main findings of this dissertation, followed by several in-depth considerations and, finally, recommendations and implications for future research and clinical practice with respect to childhood adversity in relation to mental health. 


\section{REFERENCES}

1. Rehm J, Shield KD. Global Burden of Disease and the Impact of Mental and Addictive Disorders. Curr Psychiatry Rep Feb 7 2019;21(2):10.

2. Os J van BW, van der Gaag RJ, Beekman AJ, Vermeiren R, Engels R. Laten we de diagnose schizofrenie vergeten. NRC. 7 March 2015, 2015;Opinie \& Debat: 2.

3. van Os J. "Schizophrenia" does not exist. BMJ: British Medical Journal 2016;352.

4. American Psychiatric Association. Diagnostic and Statistical Manual of Mental Disorders. 5th ed Washington, DC; 2013.

5. Tan N, van Os J. [The schizophrenia spectrum and other psychotic disorders in the DSM-5]. Tijdschr Psychiatr 2014;56(3):167-172

6. Keshavan MS, Clementz BA, Pearlson GD, Sweeney JA, Tamminga CA. Reimagining psychoses: an agnostic approach to diagnosis. Schizophr Res May 2013;146(1-3):10-16.

7. George B, Klijn A. A modern name for schizophrenia (PSS) would diminish self-stigma. Psychol Med Jul 2013;43(7):1555-1557.

8. Perala J, Suvisaari J, Saarni SI, et al. Lifetime prevalence of psychotic and bipolar I disorders in a general population. Arch Gen Psychiatry Jan 2007;64(1):19-28.

9. Guloksuz S, van Os J. The slow death of the concept of schizophrenia and the painful birth of the psychosis spectrum. Psychol Med Jan 2018;48(2):229-244.

10. van Os J, Guloksuz S. A critique of the "ultra-high risk" and "transition" paradigm. World Psychiatry Jun 2017:16(2):200-206.

11. Zubin J, Spring B. Vulnerability--a new view of schizophrenia. J Abnorm Psychol Apr 1977;86(2):103126.

12. Read J, Fink PJ, Rudegeair T, Felitti V, Whitfield CL. Child maltreatment and psychosis: A return to a genuinely integrated bio-psycho-social model. Clinical Schizophrenia and Related Psychoses 2008;2(3):235-254.

13. Dean CE. Social inequality, scientific inequality, and the future of mental illness. Philos Ethics Humanit Med Dec 19 2017;12(1):10.

14. Cockerham WC. Sociology of mental disorder. 6th ed. ed. Upper Saddle River, N.J.: Prentice Hall; 2003 .

15. Moncrieff J. Magic bullets for mental disorders: the emergence of the concept of an "antipsychotic" drug. J Hist Neurosci 2013;22(1):30-46.

16. Riese F, Guloksuz S, Roventa $\mathrm{C}$, et al. Pharmaceutical industry interactions of psychiatric trainees from 20 European countries. European Psychiatry 2015/02/01/ 2015;30(2):284-290.

17. Fusar-Poli P, Meyer-Lindenberg A. Forty years of structural imaging in psychosis: promises and truth. Acta Psychiatr Scand Sep 2016;134(3):207-224

18. Brainstorm C, Anttila V, Bulik-Sullivan B, et al. Analysis of shared heritability in common disorders of the brain. Science Jun 22 2018;360(6395).

19. Uher R, Zwicker A. Etiology in psychiatry: embracing the reality of poly-gene-environmental causation of mental illness. World Psychiatry Jun 2017:16(2):121-129.

20. McClellan J, King MC. Genetic heterogeneity in human disease. Cell Apr 16 2010;141(2):210-217.

21. Engel GL. The need for a new medical model: a challenge for biomedicine. Science Apr 8 1977:196(4286):129-136.

22. Peen J, Schoevers RA, Beekman AT, Dekker J. The current status of urban-rural differences in psychiatric disorders. Acta Psychiatr Scand Feb 2010;121(2):84-93. 
23. Cicchetti D. Resilience under conditions of extreme stress: a multilevel perspective. World Psychiatry Oct 2010:9(3):145-154.

24. Collishaw S, Pickles A, Messer J, Rutter M, Shearer C, Maughan B. Resilience to adult psychopathology following childhood maltreatment: evidence from a community sample. Child Abuse Negl Mar 2007;31(3):211-229.

25. Felitti VJ, Anda RF, Nordenberg D, Williamson DF, Spitz AM, Edwards V, Koss MP, Marks JS. Relationship of childhood abuse and household dysfunction to many of the leading causes of death in adults. The Adverse Childhood Experiences (ACE) Study. Am J Prev Med May 1998;14(4):245-258.

26. Hornor G. Childhood trauma exposure and toxic stress: what the PNP needs to know. J Pediatr Health Care Mar-Apr 2015;29(2):191-198.

27. Asmundson G. Adverse childhood experiences : using evidence to advance research, practice, policy, and prevention. 1. ed. San Deigo: Elsevier; 2019.

28. Van der Kolk BA. The body keeps the score : brain, mind, and body in the healing of trauma. New York: Viking; 2014.

29. Shonkoff JP, Garner AS, Committee on Psychosocial Aspects of C, Family H, Committee on Early Childhood A, Dependent C, Section on D, Behavioral P. The lifelong effects of early childhood adversity and toxic stress. Pediatrics Jan 2012;129(1):e232-246.

30. Aggarwal R, Deutsch JK, Medina J, Kothari N. Resident Wellness: An Intervention to Decrease Burnout and Increase Resiliency and Happiness. MedEdPORTAL Nov 6 2017;13:10651.

31. Rauschenberg C, van Os J, Cremers D, Goedhart M, Schieveld JNM, Reininghaus U. Stress sensitivity as a putative mechanism linking childhood trauma and psychopathology in youth's daily life. Acta Psychiatr Scand Oct 2017;136(4):373-388.

32. Hertzman C. Putting the concept of biological embedding in historical perspective. Proc Natl Acad Sci U S A Oct 16 2012;109 Suppl 2(Suppl 2):17160-17167.

33. Yaribeygi H, Panahi Y, Sahraei H, Johnston TP, Sahebkar A. The impact of stress on body function: A review. EXCLI J 2017:16:1057-1072.

34. Jiang S, Postovit L, Cattaneo A, Binder EB, Aitchison KJ. Epigenetic Modifications in Stress Response Genes Associated With Childhood Trauma. Front Psychiatry 2019;10:808.

35. Dedovic K, D'Aguiar C, Pruessner JC. What stress does to your brain: a review of neuroimaging studies. Can J Psychiatry Jan 2009:54(1):6-15.

36. Luijcks R, Hermens HJ, Bodar L, Vossen CJ, Van Os J, Lousberg R. Experimentally induced stress validated by EMG activity. PLoS One 2014;9(4):e95215.

37. Wijsman J, Grundlehner B, Penders J, Hermens H. Trapezius Muscle EMG as Predictor of Mental Stress. Vol 12; 2010.

38. Solomon EP, Heide KM. The biology of trauma: implications for treatment. J Interpers Violence Jan 2005;20(1):51-60.

39. Lardinois M, Lataster T, Mengelers R, Van Os J, Myin-Germeys I. Childhood trauma and increased stress sensitivity in psychosis. Acta Psychiatr Scand Jan 2011:123(1):28-35

40. Davis DA, Luecken LJ, Zautra AJ. Are reports of childhood abuse related to the experience of chronic pain in adulthood? A meta-analytic review of the literature. Clin J Pain Sep-Oct 2005;21(5):398405.

41. Sachs-Ericsson N, Blazer D, Plant EA, Arnow B. Childhood sexual and physical abuse and the 1-year prevalence of medical problems in the National Comorbidity Survey. Health Psychol Jan 2005;24(1):32-40.

42. Guinosso SA, Johnson SB, Riley AW. Multiple adverse experiences and child cognitive development. Pediatr Res Jan 2016;79(1-2):220-226. 
43. Yang L, Wang Z. Early-Life Conditions and Cognitive Function in Middle-and Old-Aged Chinese Adults: A Longitudinal Study. Int J Environ Res Public Health May 15 2020;17(10).

44. Aas M, Dazzan P, Mondelli V, Melle I, Murray RM, Pariante CM. A systematic review of cognitive function in first-episode psychosis, including a discussion on childhood trauma, stress, and inflammation. Front Psychiatry Jan 8 2014;4:182.

45. Sideli L, Fisher HL, Russo M, et al. Failure to find association between childhood abuse and cognition in first-episode psychosis patients. Eur Psychiatry Jan 2014;29(1):32-35. 




\section{CHAPTER 2}

\section{DO CURRENT MEASURES OF POLYGENIC RISK FOR MENTAL DISORDERS CONTRIBUTE TO POPULATION VARIANCE IN MENTAL HEALTH?}

\section{Published as:}

Marsman A, Pries LK, Ten Have M, de Graaf R, van Dorsselaer S, Bak M, Kenis G, Lin BD, Luykx JJ, Rutten BPF, Guloksuz S, van Os J. Do Current Measures of Polygenic Risk for Mental Disorders Contribute to Population Variance in Mental Health? Schizophr Bull. 2020; 46(6):1353-1362. 


\section{ABSTRACT}

The polygenic risk score (PRS) allows for quantification of the relative contributions of genes and environment in population-based studies of mental health. We analyzed the impact of transdiagnostic schizophrenia PRS and measures of familial and environmental risk on level of and change in general mental health (Short-Form-36 mental health) in the Netherlands Mental Health and Incidence Study-2 general population sample, interviewed 4 times over a period of 9 years, yielding 8901 observations in 2380 individuals. Schizophrenia PRS, family history, somatic pain, and a range of environmental risks and social circumstances were included in the regression model of level of and change in mental health. We calculated the relative contribution of each (group of) risk factor(s) to the variance in (change in) mental health.

In the combined model, familial and environmental factors explained around $17 \%$ of the variance in mental health, of which around $5 \%$ was explained by age and sex, $30 \%$ by social circumstances, $16 \%$ by pain, $22 \%$ by environmental risk factors, $24 \%$ by family history and $3 \%$ by PRS for schizophrenia (PRS-SZ). Results were similar, but attenuated, for the model of mental health change over time. Childhood trauma and gap between actual and desired social status explained most of the variance. PRS for bipolar disorder, cross-disorder and depression explained less variance in mental health than PRS-SZ.

Polygenic risk for mental suffering, derived from significance-testing in massive samples, lacks impact in analyses focusing on prediction in a general population epidemiological setting. Social-environmental circumstances, particularly childhood trauma and perceived status gap, drive most of the attributable variation in population mental health. 


\section{INTRODUCTION}

Heritability estimates of mental disorders, derived from twin and extended family studies, are typically in the range of $40-80 \%$. With the advent of molecular genetic testing, however, it has become clear that twin-based heritability estimates do not translate into direct effects of specific molecular genetic variation.' Molecular genetic analysis allows estimation of a model that predicts trait values from genotype data, expressed as a polygenic risk score (PRS). ${ }^{2}$ The amount of phenotypic variance explained by PRS typically is much lower than the amount of additive genetic variance estimated across twin studies. For arguably the most investigated mental disorder, schizophrenia, with an estimated twin heritability of $60-80 \%$, tens of thousands of markers explain only $7 \%$ of the variance on the liability scale and around $20 \%$ of the variance on the observed 0-1 scale derived from the logistic regression model. ${ }^{3,4}$ Thus, a considerable 'heritability gap' remains, the origin of which may represent environmental effects, indirect genetic effects within the family, rare genetic variants, gene-environment interplay, assortative mating or other factors. ${ }^{5}$

PRS is increasingly used as measure of risk, etiology, or clinical utility in epidemiological studies. ${ }^{6,7}$ In psychiatry, the PRS has been used in some epidemiological studies to examine prediction of mental disorders and related traits $^{8}$, and to test aspects of geneenvironment interplay. ${ }^{9}$ These studies, however, have mostly focused on diagnosisspecific models and not on the relative contribution of PRS in population-based models of mental health. In addition, transdiagnostic molecular genetic analyses indicate that the majority of common genetic variants are non-specifically associated with a range of mental disorders. ${ }^{10,11}$ Around two-thirds of genetic associations are common to schizophrenia, bipolar disorder and major depressive disorder and overlaps also exist with genetic variants contributing to autism, attention-deficit/hyperactivity disorder, and intellectual disabilities. 10,11 These findings suggest that polygenic risk scores for mental disorders to a large extent represent transdiagnostic risk for mental suffering. PRS for schizophrenia (PRS-SZ) in particular is associated with a variety of disorders, 12-14 quality of life, ${ }^{15}$ and subclinical multidimensional phenotypes..$^{16-23}$ Indeed, investigation of electronic health records from the United States reveal that PRS-SZ is associated with not only a diagnosis of schizophrenia but also diagnoses of other related psychiatric and medical conditions. ${ }^{13}$

Given that schizophrenia, in a transdiagnostic psychopathology perspective, can be considered as the selection at the extreme end of the mental disorder severity spectrum, PRS for schizophrenia, in comparison with other possible nonspecific PRS constructs, arguably should have the greatest probability of showing impact on mental 
health at the population level. In addition, PRS-SZ is better powered than genome wide association studies (GWAS) of any other mental disorder. The transdiagnostic perspective of PRS thus opens the way to test the basic question to what degree PRS may contribute, in a population-based setting, to variation in mental health, and how this compares to known risk factors of mental ill-health. To our knowledge, no previous study has addressed this basic question. If a transdiagnostic mental health PRS predicts mental suffering in a population-based sample, over and above traditional measures of environmental and familial risk, significant progress could be made in elucidating the role of genetics in the diagnosis and treatment of mental suffering. In addition, showing impact of PRS on mental health in population-based, epidemiological settings would considerably increase the scope for preventative usage of PRS.

The expectation, guided the existing literature, ${ }^{8}$ is that measures of PRS will have little or no predictivity in an epidemiological setting, as their contribution typically is evaluated on the basis of statistical significance-testing in massive samples, in which minute effects can acquire statistical significance. ${ }^{24}$ For prediction in a general population, epidemiological setting, however, a minimum clinical effect size is required to generate a statistical signal. ${ }^{24}$

As this aspect of PRS has not been analyzed previously in an epidemiological setting, in comparison with established clinical predictors, this study set out to comparatively quantify prediction of PRS in a general population setting. To this end, we examined the contribution of PRS for schizophrenia (PRS-SZ) and other known risk factors to the variance in level and change of mental health in a large population-based cohort that was examined 4 times over period of 9 years. Guided by previous work in this sample, we used a mental health phenotype that was responsive to variation in PRS-SZ. ${ }^{25}$ 


\section{METHODS}

\section{Study Population}

All 4 waves of the Netherlands Mental Health Survey and Incidence Study-2 (NEMESIS-2) were used. NEMESIS-2 was conducted to study the prevalence, incidence, course, and consequences of mental disorders in the Dutch general population. The baseline data of NEMESIS-2 were collected from 2007 to 2009, and the follow-up was until 2018. The study was approved by the Medical Ethics Review Committee for Institutions on Mental Health Care and written informed consent was collected from participants at each wave. To ensure representativeness of the sample in terms of age (between the ages of 18 and 65 at baseline), region, and population density, a multistage random sampling procedure was applied. Dutch illiteracy was an exclusion criterion. Non-clinician, trained interviewers applied the Composite International Diagnostic Interview (CIDI) version 3.026,27 and additional questionnaires during home visits. Details of NEMESIS-2 are provided elsewhere..$^{28,29}$ The first wave ( $T_{0}$ ) enrolled 6646 participants (response rate 65.1\%; average interview duration: 95 minutes), who were followed up in 3 visits within 9 years: successive response rates at year $3\left(T_{1}\right)$, year $6\left(T_{2}\right)$, and year $9\left(T_{3}\right)$ were $80.4 \%$ ( $n=5303$; excluding those who deceased; interview duration: 84 minutes), 87.8\% ( $n=4618$; interview duration: 83 minutes), and 86.8\% ( $n=4007$; interview duration: 102 minutes), respectively. Thus, more than $60 \%$ of the sample had follow-up from baseline to $T_{3}$. Rates at baseline reflect lifetime occurrence; rates at $T_{1}$ to $T_{3}$ reflect approximately 3-year interval (baseline- $T_{1}, T_{1}-T_{2}$, and $T_{2}-T_{3}$ ) occurrence. Attrition between $T_{0}$ and $T_{3}$ was not significantly associated with any of the individual 12 -month mental disorders at $T_{0}$ after controlling for sociodemographic characteristics. ${ }^{30,31}$

\section{Measurements}

Mental health

The Short-Form-36 (SF-36) Health Survey ${ }^{32}$ consists of 8 subscales, each scale ranging from poor (0) to good (100) functioning. Mental health, role limitations due to emotional problems, social functioning, and vitality were averaged into a single mental health dimension, while general health perceptions, physical functioning, role limitation due to physical health problems, and bodily pain were averaged into the physical health dimension. ${ }^{33}$ The SF-36 was assessed at each time-point and refer to the past 4 weeks. As per previous work in this sample examining PRS, 25 the SF-36 mental health dimension at each time point was used in the analyses as the dependent variable, scored reversely so that higher scores reflect less mental health. In addition, the SF-36 dimension of bodily pain was used as an independent variable, given the fact that: mental ill-health and pain are strongly associated with 
each other; pain affects between one-third and one-half of the population; and pain represents one of the most prominent causes of disability worldwide according to the Global Burden of Disease reviews. ${ }^{34-36}$

\section{Adverse Social Circumstances}

Age was expressed in years, sex was coded male ('O') or female ('1'). Marital status at each interview was coded married/widowed versus divorced/never married. Unemployment at each interview was coded as having no employment versus employment/ homemaker/student/retired. Educational level at baseline was a 4-level variable (primary, lower and higher secondary, and higher professional/university education); income at each interview was net annual household income, rated on a scale from 1 to 14 (not rated at one interview and predicted linearly from the values at the interviews before and after). Having ever been on disability benefit over the period of observation was analyzed as a binary variable (5\% of the sample). The variable 'debts' was rated present at each interview (not rated at one interview and predicted linearly from the values at the interviews before and after) if the participant had arrears in payment or acquired debts. The variable 'living alone' at each interview indicated that the participant was the only person in the household. The perceived status gap was assessed at $T_{1}, T 2$ and $T_{3}$ using two questions. First, the MacArthur Scale of Subjective Social Status ${ }^{37}$ was used to rate subjective social status. In an easy pictorial format, it presents a 'social ladder' with 10 steps and asks individuals to place an ' $X$ ' on the step on which they feel they stand. The second question was about a similar ladder, but this time with regard to the desired level of social status. The difference between the subjective desired and actual social status was used as independent variable in the analyses. It was treated as a person-level variable in the analyses.

\section{Family History and Parental History}

Family history was assessed as a person-level characteristic across 2 variables, as described in a previous publication. ${ }^{38}$ First, for participants who screened positive for the following psychiatric diagnoses, presence of the disorder in direct relatives was assessed at each interview wave: alcohol/drugs abuse/dependence, depression, mania, and anxiety disorders (panic disorder, social phobia, agoraphobia, generalized anxiety disorder). This variable will be referred to as 'family history'. A total of 51\% of the sample screened positive for this variable at any of the 4 interview waves. Second, at $T_{1}$, self-reported parental history of 'problems with alcohol', 'problems with drugs', 'any psychiatric treatment or admission', 'severe anxiety or phobias', 'severe depression', 'suicide', and 'delusions or hallucinations' were assessed in the entire sample. A total of $31 \%$ screened positive for positive parental family history. This variable will be referred to as 'parental history'. 


\section{Childhood adversity}

Childhood adversity was assessed at $T_{0}$ using a questionnaire based on the NEMESIS trauma questionnaire. ${ }^{28}$ Whenever a subject reported having experienced 1 of 5 types of childhood adversity before the age of 16 years (emotional neglect [not listened to, ignored, or unsupported], physical abuse [kicked, hit, bitten, or hurt with object or hot water], psychological abuse [yelled at, insulted, unjustly punished/ treated, threatened, belittled, or blackmailed], peer victimization [bullying], and one time or more sexual abuse [any unwanted sexual experience]), they were asked to state how often it had occurred. The item 'sexual abuse' was rated on a scale of 1 (once) to 5 (very often), while all other items (namely, emotional neglect, physical abuse, psychological abuse, and peer victimization or bullying) were rated and on a scale of 1 (sometimes) to 4 (very often). The total childhood adversity score was used in the analyses.

\section{Cannabis Exposure}

Lifetime cannabis use was assessed with the section substance use disorders of the CIDI 3.0 at baseline ( $T_{0}$ ). If subjects reported cannabis use, they were rated on frequency of use in the period of most frequent use on a scale of 1 (never) to 7 (every day). Consistent with previous work, ${ }^{38,39}$ a binary variable (absent = ' 0 ' and present = '1') was constructed by using the cutoff value of once per week or more in the period most frequent use.

\section{Urbanicity}

The extent of the exposure to urban environment until age 16 years was constructed at 5 levels based on the Dutch classification of population density: (1) countryside (distances to amenities is larger), (2) village (<25 000 inhabitants), (3) small city (25 000-50 000 inhabitants), (4) medium city (50 000-100 000 inhabitants), (5) large city (>100 000 inhabitants).

\section{Adulthood stressful life events}

Based on the 'Brugha Life events section,'40 participants were asked at each interview whether they experienced 1 of 9 life events within the last 12 months ( $T_{0}$ ) or since the last interview $\left(T_{1}-T_{3}\right)$. Examples of items are serious sickness, death of family member or close friend, and serious financial problems. The continuous life event score at the 4 interview occasions was used in the analyses.

\section{Polygenic Risk Score for Schizophrenia}

PRS-SZ was created from best-guess genotypes at 6 different p-thresholds $\left(.5, .1, .05,5 \times 10^{-3}, 5 \cdot 10^{-5}, 5 \cdot 10^{-8}\right)$. For our primary analyses, we used the $p$-threshold of $<.05$, as this threshold explained most variation in the phenotype in the Psychiatric 
Genomics Consortium analysis ${ }^{41}$ and was previously shown to perform well for the current phenotype of SF-36 mental health. ${ }^{42}$ For details on the genotyping, see the supplementary material. Statistical analyses were adjusted for three principal components.

Use of schizophrenia polygenic risk as transdiagnostic measure

We used PRS-SZ as a measure of transdiagnostic genetic liability. In explaining 7\% of the variance on the liability scale, PRS-SZ clearly outperforms the rest of the PRSs for mental disorder phenotypes that have been estimated so far and appears to be the forerunner for developing PRS-based clinical applications. ${ }^{13}$

In a sensitivity analysis, we also examined results using the following other PRS: PRS bipolar disorder, PRS educational achievement, PRS cross-disorder, PRS IQ and PRS MDD, 10,43-45 and finally we examined a model with the joint multivariable contribution of all PRS that contributed in univariable models.

\section{Statistical analyses}

Risk set

Material for DNA analysis of sufficient quality was available for 3104 individuals (47\%) at $T_{0}$ (see supplementary material). Excluding individuals who at interview had been assessed as member of an ethnic minority, given lack of generalizability of polygenic risk scores in this group, left 3052 participants. Of the 3052, 2380 had non-missing values for all variables used in the analyses, yielding 8901 observations over the four interviews. Values for all variables were very similar in a comparison between the 8901 included and the 10127 non-included observations (table 1).

\section{Analyses}

All analyses were performed using Stata, version $16 .{ }^{46} \mathrm{P}<.05$ (2-tailed) was considered nominally statistically significant. We fitted cross-sectional regression models, adjusted for time, to test the effects of the independent variables on mental health as dependent variables. As each person contributed four observations in the cross-sectional model, the data were hierarchically structured. The Stata cluster option was therefore used to take into account intra-group correlations occasioned by clustering of observations within individuals. Some variables were assessed at each time-point and therefore timevarying; other variables were demographics or antecedents and time-invariant. Models including PRS-SZ were adjusted for 3 principal components. Shapely decomposition (Stata shapley2 command) was used to calculate the relative contribution of each (group of) regressor(s) to the $R^{2}$ statistic. 
The contribution of each (group of) regressor(s) to the model was statistically evaluated using likelihood ratio tests with the Stata test postestimation command.

Regressor groups (jointly) evaluated were: (1) PRS; (2) family history and parental history (family history); (3) urbanicity, cannabis use, childhood trauma and life events (environmental risks); (4) somatic pain; and (5) living alone, no partner, unemployment, household income, educational status, perceived status gap, received disability and debts (social circumstances).

Analyses were conducted separately for (1) level of mental health: cross-sectional analysis of the 4 measures of mental health, and (2) change in mental health which was similar to (1) but with adjustment for the baseline value of mental health, thus effectively assessing the effect of predictors of change of mental health over time.

Models were developed by adding more groups of variables across 5 steps. In addition, we calculated, in separate regression analyses, the standardized effect sizes (beta) and contributions to explained variance of all the individual factors in the regressor groups. 


\section{RESULTS}

Sample characteristics and representativeness are shown in table 1. Of the participants included in the analysis, mean age was 50.0 years (SD = 12.7), 56\% was female. Distributions of variables did not differ between participants included and excluded of analysis. Results are summarized in tables 2 and 3 and figure 1.

\section{Level of mental health}

PRS-SZ contributed significantly to mental health across all models; however, the relative contribution to $R^{2}$ was very small. In the combined model (table 2; model 5), proxy genetic and environmental factors explained around $17 \%$ of the variance in mental health, of which around 5\% was explained by age and sex, 30\% by social circumstances, $16 \%$ by pain, $22 \%$ by environmental risk factors, $24 \%$ by family history, and $3 \%$ by PRS-SZ (figure 1).

Of the environmental risks, childhood trauma had the largest impact, followed by life events, whereas urbanicity and cannabis use did not contribute significantly. Of the social circumstances, perceived status gap had the largest impact, although other variables contributed comparatively, with the exception of unemployment which did not contribute. Of the variables age and sex, only sex contributed significantly (table 3).

The effect of family history was not reducible to PRS-SZ (only $2 \%$ reduction; model 3). In contrast, the contribution of the PRS-SZ was reduced by $20 \%$ when the family history variables were added to the model (table 2). The contributions of both 'family history' and PRS-SZ were reduced by around 30\% from the model with only time and age/sex, to the full model with all independent variables (table 2). Conversely, the contributions of environmental risks and social circumstances were not affected much by adding PRS-SZ and family history information to the model (table 2)

\section{Change in mental health}

PRS-SZ did not contribute significantly to mental health in any of the models of mental health change (table 2). In the model of mental health change over time, proxy genetic and environmental factors explained around $12 \%$ of the variance, of which around 6\% was explained by age and sex, 29\% by social circumstances, $17 \%$ by pain, $24 \%$ by environmental risk factors, $24 \%$ by family history and $2 \%$ by PRS$\mathrm{SZ}$, the latter not statistically significant (figure 1). Of the different environmental risks, childhood trauma had the largest impact, followed by life events, whereas 
urbanicity and cannabis use did not contribute significantly. Of the different social circumstances, having received disability benefit had the largest impact, although other variables contributed comparatively, with the exception of educational level, having no partner, household income and unemployment. Both age and sex contributed significantly (table 3).

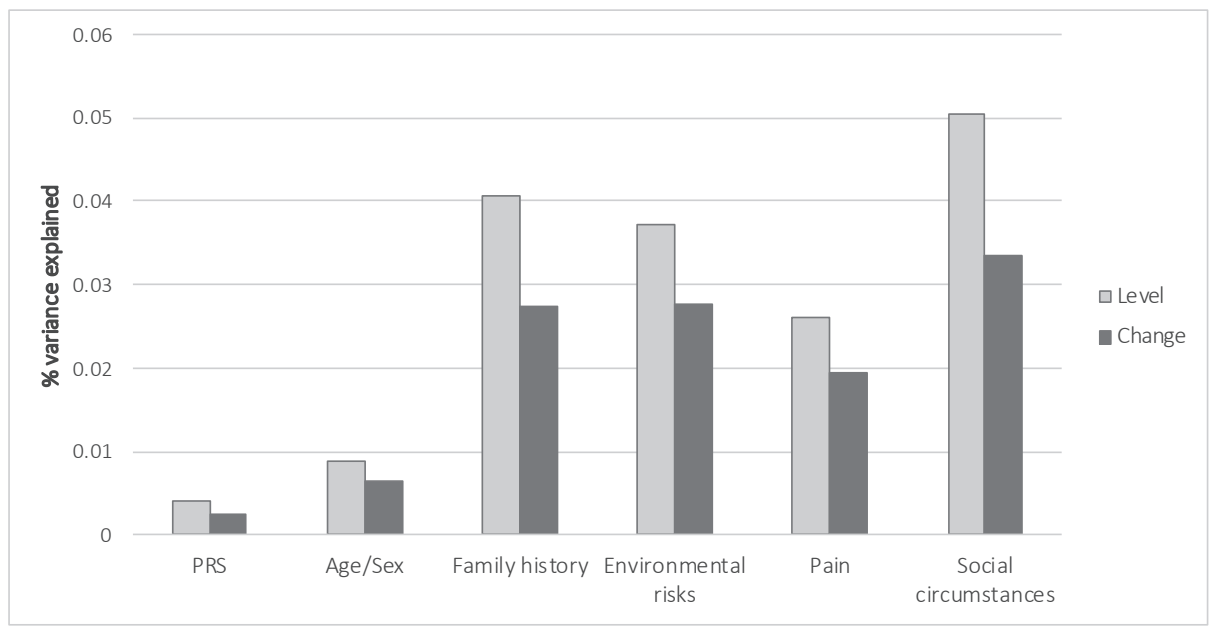

Figure 1. Contribution of factors used to explain mental health variance in models of level of mental health and change of mental health (all statistically significant except PRS in model of change)

\section{Sensitivity analyses}

PRS depression, PRS bipolar disorder, and PRS cross-disorder contributed less than PRS-SZ. PRS educational achievement and PRS-IQ did not contribute at all (table 4). The different PRS only marginally added to each other: the multivariable contribution of PRS depression, PRS bipolar disorder, PRS cross-disorder, and PRS-SZ rose from $0.4 \%$ to $0.6 \%$ in the full model of level of mental health, and from $0.2 \%$ to $0.4 \%$ in the full model of change of mental health (tables 2 and 4 ). 
Table 1. Sample characteristics, stratified by risk set included for analysis ( $n=8901$ observations) or excluded from analysis ( $n=10127$ observations)

\begin{tabular}{lcccccccc}
\hline Sample & PRS & $\begin{array}{c}\text { Parental } \\
\text { history }\end{array}$ & $\begin{array}{c}\text { Family } \\
\text { history } \\
\%\end{array}$ & $\begin{array}{c}\text { Childhood } \\
\text { trauma } \\
\text { Mean }\end{array}$ & $\begin{array}{c}\text { Regular } \\
\text { cannabis use }\end{array}$ & $\begin{array}{c}\text { Urbani- } \\
\text { city } \\
\text { civing }\end{array}$ & $\begin{array}{c}\text { Life } \\
\text { alone } \\
\%\end{array}$ & $\begin{array}{c}\text { Mean } \\
\text { events } \\
\text { Mean }\end{array}$ \\
\hline Excluded & -130.95 & 0.31 & 0.52 & 0.2 & 0.02 & 2.99 & 0.73 & 0.22 \\
SD & 4.48 & & & 0.4 & & 1.34 & & 0.41 \\
N & 1081 & 8979 & 10127 & 10127 & 9590 & 10106 & 10009 & 10127 \\
\hline Included & -131.29 & 0.31 & 0.53 & 0.19 & 0.02 & 2.99 & 0.68 & 0.18 \\
SD & 4.33 & & & 0.39 & & 1.34 & 0.39 \\
N & 8901 & 8901 & 8901 & 8901 & 8901 & 8901 & 8901 & 8901 \\
\hline Total & -131.25 & 0.31 & 0.52 & 0.2 & 0.02 & 2.99 & 0.71 & 0.2 \\
SD & 4.35 & & & 0.4 & & 1.34 & 0.4 \\
N & 9982 & 17880 & 19028 & 19028 & 18491 & 19007 & 18910 & 19028 \\
\hline
\end{tabular}

Table 2. Contributions of proxy genetic and non-genetic risksa to level of and change in mental health

\begin{tabular}{lll}
\hline Level of mental health & Model 1 (\%) & Model 2 (\%) \\
\hline Time & 1.8 & 1.8 \\
PRS & 0.6 & 1.2 \\
Age/sex & 1.7 & 6.1 \\
Family history & & \\
Environmental risks & & \\
Pain & & 7.3 \\
Social circumstances & 2.3 & Model 2 (\%) \\
\hline Total & Model 1 (\%) & 1.8 \\
\hline Change in mental health & 1.8 & 0.8 \\
Time & $0.3^{*}$ & 3.7 \\
PRS & 1.0 & \\
Age/sex & & \\
Family history & & 4.5 \\
Environmental risks & & \\
Pain & 1.3 & \\
Social circumstances & & \\
\hline Total & & \\
\hline
\end{tabular}

Note. Model 1: PRS-SZ only; model 2: family history only; model 3: PRS-SZ and family history; model 4: environmental risks (childhood trauma, regular cannabis use, urban environment), pain and social circumstances (living alone, jobless, income, educational level, recent life events, no partner, perceived status gap, disability payment, debts); model 5: all factors of models 3 and 4 combined; \% model 5: as percentage of total variance explained. 


\begin{tabular}{|c|c|c|c|c|c|c|c|c|c|}
\hline $\begin{array}{c}\text { No } \\
\text { partner }\end{array}$ & $\begin{array}{l}\text { Unemploy- } \\
\text { ment }\end{array}$ & Income & $\begin{array}{l}\text { Edu- } \\
\text { cation }\end{array}$ & $\begin{array}{l}\text { Status } \\
\text { gap }\end{array}$ & $\begin{array}{l}\text { Disa- } \\
\text { bility }\end{array}$ & Pain & Debts & Age & $\begin{array}{c}\text { Female } \\
\text { Sex }\end{array}$ \\
\hline$\%$ & $\%$ & Mean & Mean & Mean & $\%$ & Mean & $\%$ & Mean & $\%$ \\
\hline \multirow[t]{2}{*}{0.39} & 0.12 & 6.86 & 2.99 & 0.95 & 0.05 & 0.1 & -84.39 & 48.59 & 0.54 \\
\hline & & 2.49 & 0.9 & 1.33 & & 0.3 & & 13.1 & \\
\hline 10126 & 10127 & 9364 & 10127 & 8756 & 10119 & 8959 & 10120 & 10127 & 10127 \\
\hline \multirow[t]{2}{*}{0.35} & 0.12 & 7.08 & 3.07 & 0.8 & 0.04 & 0.1 & -84.46 & 48.98 & 0.56 \\
\hline & & 2.4 & 0.88 & 1.15 & & 0.29 & & 12.71 & \\
\hline 8901 & 8901 & 8901 & 8901 & 8901 & 8901 & 8901 & 8901 & 8901 & 8901 \\
\hline \multirow[t]{2}{*}{0.37} & 0.12 & 6.97 & 3.03 & 0.87 & 0.05 & 0.1 & -84.43 & 48.77 & 0.55 \\
\hline & & 2.45 & 0.89 & 1.24 & & 0.3 & & 12.92 & \\
\hline 19027 & 19028 & 18265 & 19028 & 17657 & 19020 & 17860 & 19021 & 19028 & 19028 \\
\hline
\end{tabular}

\begin{tabular}{llll}
\hline Model 3(\%) & Model 4 (\%) & Model 5 (\%) & \% Model 5 (\%) \\
\hline 1.8 & 1.8 & 1.8 & 2.5 \\
0.5 & & 0.4 & 5.3 \\
1.2 & 1.1 & 0.9 & 24.3 \\
6.0 & & 4.1 & 22.2 \\
& 4.5 & 3.7 & 15.6 \\
& 2.9 & 2.6 & 30.2 \\
\hline 7.7 & 5.5 & 5.1 & \\
\hline Model 3(\%) & 14.0 & 16.7 & \%odel 5 (\%) \\
1.8 & Model 4 (\%) & Model 5 (\%) & 2.1 \\
$0.3 *$ & 1.8 & 1.8 & 5.5 \\
0.8 & & $0.2 *$ & 23.4 \\
3.7 & 0.7 & 0.6 & 23.7 \\
& & 2.7 & 16.8 \\
& 3.2 & 2.8 & 28.6 \\
\hline 4.7 & 2.1 & 2.0 & \\
\hline
\end{tabular}

All associations with regressor groups are statistically significant except marked with *.

a Contributions of genetic principal components not displayed.

b Excludes contribution of factor 'time'. 
Table 3. Individual factor effect size and variance explained

Table 2; model 5*

Level of mental health

Change in mental health

$\begin{array}{lllllll}\text { Beta } t & p & R^{2} & \text { Beta } t & p & R^{2}\end{array}$

(\%) (\%)

\begin{tabular}{|c|c|c|c|c|c|c|c|c|c|}
\hline PRS & PRS & 0.054 & 3.702 & 0.000 & 0.4 & 0.015 & 1.554 & 0.120 & 0.3 \\
\hline \multirow[t]{2}{*}{ Family history } & Parental history & 0.044 & 2.938 & 0.003 & 0.8 & 0.026 & 2.369 & 0.018 & 0.6 \\
\hline & Family history & 0.147 & 10.560 & 0.000 & 3.6 & 0.069 & 7.052 & 0.000 & 2.5 \\
\hline \multirow{4}{*}{$\begin{array}{l}\text { Environ- } \\
\text { mental } \\
\text { risks }\end{array}$} & Childhood trauma & 0.095 & 4.825 & 0.000 & 2.3 & 0.049 & 3.788 & 0.000 & 1.6 \\
\hline & Regular cannabis use & 0.017 & 1.309 & 0.191 & 0.1 & 0.007 & 0.849 & 0.396 & 0.1 \\
\hline & Urbanicity & -0.005 & -0.367 & 0.713 & 0.0 & -0.008 & -0.728 & 0.467 & 0.0 \\
\hline & Life events & 0.096 & 8.405 & 0.000 & 1.5 & 0.077 & 8.283 & 0.000 & 1.2 \\
\hline \multirow{8}{*}{$\begin{array}{l}\text { Social } \\
\text { circum- } \\
\text { stances }\end{array}$} & Living alone & 0.077 & 4.912 & 0.000 & 1.0 & 0.040 & 3.196 & 0.001 & 0.7 \\
\hline & No partner & 0.043 & 2.666 & 0.008 & 0.8 & -0.001 & -0.104 & 0.917 & 0.6 \\
\hline & Unemployed & 0.009 & 0.648 & 0.517 & 0.6 & 0.002 & 0.198 & 0.843 & 0.5 \\
\hline & Income & -0.037 & -2.476 & 0.013 & 0.3 & -0.015 & -1.369 & 0.171 & 0.2 \\
\hline & Educational level & 0.047 & 2.941 & 0.003 & 0.1 & 0.021 & 1.856 & 0.063 & 0.0 \\
\hline & Perceived status gap & 0.070 & 3.760 & 0.000 & 1.1 & 0.025 & 1.893 & 0.058 & 0.8 \\
\hline & Disability & 0.075 & 3.672 & 0.000 & 1.2 & 0.039 & 2.541 & 0.011 & 0.9 \\
\hline & Debts & 0.049 & 3.596 & 0.000 & 0.8 & 0.035 & 3.483 & 0.000 & 0.6 \\
\hline Somatic pain & Pain & 0.121 & 8.183 & 0.000 & 2.6 & 0.079 & 6.589 & 0.000 & 1.9 \\
\hline \multirow[t]{2}{*}{ Age/sex } & Age & -0.026 & -1.572 & 0.116 & 0.1 & -0.028 & -2.315 & 0.021 & 0.1 \\
\hline & Female sex & 0.059 & 4.266 & 0.000 & 0.8 & 0.028 & 2.807 & 0.005 & 0.5 \\
\hline
\end{tabular}

Note. Beta: standardized regression coefficient; t: test statistic t; p: p-value; $R^{2}$ : percentage variance explained.

*The sum of the $R^{2}$ of individual factors may not correspond exactly to the combined $R^{2}$ in table 2 because of small differences in Stata shapley2 model specification. 


\section{DISCUSSION}

\section{Summary of findings}

The results of this study suggest that the transdiagnostic PRS-SZ is associated crosssectionally with a phenotype of mental health in the general population, in line with emerging work showing small statistical associations between PRS and various mental health phenotypes in the general population. ${ }^{8}$ PRS-SZ was not associated with change in mental health over time.

Contrary to case control studies, however, in which the PRS-SZ explains a proportion of the variance of the latent liability (7\%) or the observed scale (20\%), 3,4 the contribution of PRS-SZ to the variance of mental health was very small in the cross-sectional and non-significant in the change model of mental health. The lack of contribution of PRSSZ contrasted sharply with traditional measures of familial and environmental risk; socio-environmental circumstances were responsible for the bulk of the explained variance, particularly childhood trauma and perceived status gap.

\section{Interpretation of findings}

These results cannot be interpreted as showing that genetic factors are not important. Indeed, all measures of environmental and social circumstances that were used may in fact reflect, to a degree, genetic effects. ${ }^{47}$ Conversely, measures of family history also mediate environmental effects such as higher rates of birth and pregnancy complications, ${ }^{48-50}$ growing up in an unfavorable home environment, ${ }^{51}$ out-of-home placement. ${ }^{52}$ elevated divorce rate, alterations in parental communication, ${ }^{53}$ altered school functioning, ${ }^{54}$ and the psychosocial impact of growing up with a parent with mental illness. ${ }^{55}$ What the results do indicate, however, is that current transdiagnostic measures of polygenic risk lack impact in epidemiological general population studies, beyond very small but statistically significant associations. Genetic factors may contribute to variance of mental health in population-based samples, but it appears they are not captured by the current version of various transdiagnostic PRS.

The effect of family history was not reducible to PRS. This is compatible with previous work showing that in psychotic disorder, only a fraction of the effect of family history is mediated by PRS. ${ }^{56}$

Some environmental factors, such as cannabis, did not predict in the multivariable model, which may be considered unexpected. However, in a post-hoc univariable model, cannabis did contribute strongly ( $p=.007$ ); adding other environmental risks indicated that some of its effect was reducible to other variables, such as childhood trauma. 
Table 4. Sensitivity analysis with cross-disorder polygenic score: contributions of proxy genetic and non-genetic risks to level of and change in mental health

\begin{tabular}{llllll}
\hline Level of mental health & $\begin{array}{l}\text { Model 1 } \\
\text { (\%) }\end{array}$ & $\begin{array}{l}\text { Model } 2 \\
\text { Podel 3 }\end{array}$ & $\begin{array}{l}\text { Model 4 } \\
\text { (\%) }\end{array}$ & $\begin{array}{l}\text { Model 5 } \\
\text { (\%) }\end{array}$ \\
\hline PRS schizophrenia & 0.6 & -- & 0.5 & -- & 0.4 \\
PRS cross-disorder & 0.2 & -- & 0.1 & -- & 0.1 \\
PRS bipolar disorder & 0.2 & -- & 0.1 & -- & 0.1 \\
PRS depression & 0.4 & -- & 0.3 & -- & 0.2 \\
PRS IQ & 0.0 & -- & 0.0 & -- & 0.0 \\
PRS educational achievement & 0.0 & -- & 0.0 & -- & 0.0 \\
PRS depression/bipolar/schizophrenia / & 1.0 & -- & 0.7 & -- & 0.6 \\
cross-disorder entered together & & & & & \\
\hline Change in mental health & & & & -- & 0.2 \\
\hline PRS schizophrenia & 0.3 & -- & 0.3 & -- & 0.1 \\
PRS cross-disorder & 0.1 & -- & 0.1 & -- & 0.1 \\
PRS bipolar disorder & 0.1 & -- & 0.1 & -- & 0.1 \\
PRS depression & 0.2 & -- & 0.2 & -- & 0.0 \\
PRS IQ & 0.0 & -- & 0.0 & -- & 0.0 \\
PRS educational achievement & 0.0 & -- & 0.0 & -- & 0.4 \\
PRS depression/bipolar/ schizophrenia / & 0.6 & -- & 0.5 & -- & \\
cross-disorder entered together & & & & & \\
\hline
\end{tabular}

Note. Model 1: PRS only; model 2: Family history only; model 3: PRS and family history; model 4: Environmental risks (childhood trauma, regular cannabis use, urban environment), pain and social circumstances (living alone, jobless, income, educational level, recent life events, no partner, perceived status gap, disability payment, debts); model 5: all factors of models 3 and 4 combined.

The lack of relevance of PRS-SZ is not related to the choice of phenotype, as associations between PRS-SZ and the range of mental health phenotypes used to date, similarly are very small although sometimes showing statistical significance. ${ }^{16-23}$

\section{The contribution of epidemiological predictors}

There is a large literature on the impact of environmental risks and social circumstances on mental health, and how this may inform policy. ${ }^{57}$ Our results do not suggest that traditional socio-environmental risks are reducible to the genetic factors that are captured by transdiagnostic polygenic risk, although genes and environment may show a degree of synergistic interaction. ${ }^{9}$ The results are compatible with the suggestion that mental health and mental health research may be productively approached from the perspective of public health. ${ }^{58}$ In addition, pain was confirmed as major factor impacting health, as expected given its strong association with mental health, high prevalence and prominent contribution to disability worldwide. ${ }^{34-36}$ 


\section{Methodological issues}

The predictivity of even the full model of mental health was low at less than $20 \%$. This, however, is conform expectation in the domain of behavioral and mental science, where predictivity of models typically is limited. ${ }^{59}$

It could be argued that modelling other phenotypes for PRS analysis would be more productive. This is unlikely, however, as previous work examining associations between PRS-SZ and a range of mental disorders and associated trait phenotypes has shown similar weak and ambiguous associations..$^{16-23}$ Given the comorbid nature of psychopathology, it is highly unlikely that PRS-SZ would show robust associations with another, hitherto untested phenotype.

Similarly, we showed that other measures of PRS did not improve PRS performance and that different PRS only minimally added to each other. The analyses included less than half of the original sample. However, it is unlikely that this would have resulted in bias as there was no evidence of differential attrition from analysis.

\section{Conclusion}

These findings suggest that the examination of molecular genetic risk for mental suffering, derived from theoretical analyses focusing on significance-testing, lack impact in analyses focusing on prediction in epidemiological settings. ${ }^{24}$

\section{Supplementary material}

Supplementary material is available at Schizophrenia Bulletin.

\section{Funding}

Financial support for this study has been received from the Ministry of Health, Welfare and Sport, with supplementary support from the Netherlands Organization for Health Research and Development (ZonMw). This work was supported by the European Community's Seventh Framework Program under grant agreement No. HEALTH-F2-2009-241909 (Project EU-GEI). B.P.F.R. Rutten was funded by a VIDI award number 91718336 from the Netherlands Scientific Organisation.

\section{Acknowledgments}

NEMEIS-2 is conducted by the Netherlands Institute of Mental Health and Addiction (Trimbos Institute) in Utrecht. The funder had no role in the design and conduct of the study; collection, management, analysis, and interpretation of the data; preparation, review, or approval of the manuscript; and decision to submit the manuscript for publication. 


\section{REFERENCES}

1. Kendler KS. A joint history of the nature of genetic variation and the nature of schizophrenia. Mol Psychiatry Feb 2015:20(1):77-83.

2. Purcell SM, Wray NR, Stone JL, et al. Common polygenic variation contributes to risk of schizophrenia and bipolar disorder. Nature Jul 12009.

3. Pardinas AF, Holmans P, Pocklington AJ, et al. Common schizophrenia alleles are enriched in mutation-intolerant genes and in regions under strong background selection. Nat Genet Mar 2018;50(3):381-389.

4. Ripke S, O'Dushlaine C, Chambert K, et al. Genome-wide association analysis identifies 13 new risk loci for schizophrenia. Nat Genet Oct 2013;45(10):1150-1159.

5. Young Al. Solving the missing heritability problem. PLoS Genet Jun 2019;15(6):e1008222.

6. Calafato MS, Thygesen JH, Ranlund S, et al. Use of schizophrenia and bipolar disorder polygenic risk scores to identify psychotic disorders. Br J Psychiatry Sep 2018;213(3):535-541.

7. Schijven D, Veldink JH, Luykx JJ. Genetic cross-disorder analysis in psychiatry: from methodology to clinical utility. Br J Psychiatry May 2020;216(5):246-249.

8. Martin AR, Daly MJ, Robinson EB, Hyman SE, Neale BM. Predicting Polygenic Risk of Psychiatric Disorders. Biol Psychiatry Jul 15 2019;86(2):97-109.

9. Guloksuz S, Pries LK, Delespaul P, et al. Examining the independent and joint effects of molecular genetic liability and environmental exposures in schizophrenia: results from the EUGEl study. World Psychiatry Jun 2019:18(2):173-182.

10. Cross-Disorder Group of the Psychiatric Genomics Consortium. Genomic Relationships, Novel Loci, and Pleiotropic Mechanisms across Eight Psychiatric Disorders. Cell Dec 12 2019;179(7):1469-1482 e1411.

11. Cross-Disorder Group of the Psychiatric Genomics C, Lee SH, Ripke S, et al. Genetic relationship between five psychiatric disorders estimated from genome-wide SNPs. Nat Genet Sep 2013:45(9):984-994.

12. Richardson TG, Harrison S, Hemani G, Davey Smith $G$. An atlas of polygenic risk score associations to highlight putative causal relationships across the human phenome. Elife Mar 5 2019;8:e43657.

13. Zheutlin AB, Dennis J, Karlsson Linner R, et al. Penetrance and Pleiotropy of Polygenic Risk Scores for Schizophrenia in 106,160 Patients Across Four Health Care Systems. Am J Psychiatry Oct 1 2019:176(10):846-855.

14. Mistry S, Harrison JR, Smith DJ, Escott-Price V, Zammit S. The use of polygenic risk scores to identify phenotypes associated with genetic risk of schizophrenia: Systematic review. Schizophr Res Jul 2018;197:2-8.

15. Pazoki R, Lin BD, van Eijk KR, Schijven D, Guloksuz S, GROUP investigators, Luykx JJ. Polygenic risk scores are associated with quality of life in schizophrenia. submitted.

16. Hatzimanolis A, Bhatnagar $P$, Moes A, et al. Common genetic variation and schizophrenia polygenic risk influence neurocognitive performance in young adulthood. American Journal of Medical Genetics B - Neuropsychiatric Genetics Jul 2015;168B(5):392-401.

17. Jones HJ, Stergiakouli E, Tansey KE, et al. Phenotypic Manifestation of Genetic Risk for Schizophrenia During Adolescence in the General Population. JAMA psychiatry Mar 2016;73(3):221-228.

18. Mistry S, Harrison JR, Smith DJ, Escott-Price V, Zammit S. The use of polygenic risk scores to identify phenotypes associated with genetic risk of schizophrenia: Systematic review. Schizophr Res Nov 92017. 
19. Riglin L, Collishaw S, Richards A, Thapar AK, Maughan B, O'Donovan MC, Thapar A. Schizophrenia risk alleles and neurodevelopmental outcomes in childhood: a population-based cohort study. The lancet Psychiatry Jan 2017;4(1):57-62.

20. Nivard MG, Gage SH, Hottenga JJ, et al. Genetic Overlap Between Schizophrenia and Developmental Psychopathology: Longitudinal and Multivariate Polygenic Risk Prediction of Common Psychiatric Traits During Development. Schizophr Bull Oct 21 2017;43(6):1197-1207.

21. Power RA, Steinberg S, Bjornsdottir G, et al. Polygenic risk scores for schizophrenia and bipolar disorder predict creativity. Nat Neurosci Jul 2015;18(7):953-955.

22. Hatzimanolis A, Avramopoulos D, Arking DE, et al. Stress-Dependent Association Between Polygenic Risk for Schizophrenia and Schizotypal Traits in Young Army Recruits. Schizophr Bull Feb 15 2018;44(2):338-347.

23. van Os J, Pries LK, Delespaul P, et al. Replicated evidence that endophenotypic expression of schizophrenia polygenic risk is greater in healthy siblings of patients compared to controls, suggesting gene-environment interaction. The EUGEl study. Psychol Med Aug 15 2019:1-14.

24. Lo A, Chernoff H, Zheng T, Lo SH. Why significant variables aren't automatically good predictors. Proc Natl Acad Sci U S A Nov 10 2015;112(45):13892-13897.

25. Pries $L$, van Os J, ten Have $M$, et al. The main and interacting effects of recent stressful life events with genomic and exposomic liability for schizophrenia on mental and physical health: a prospective cohort study. . Submitted Manuscript 2020.

26. Alonso J, Angermeyer MC, Bernert S, et al. Sampling and methods of the European Study of the Epidemiology of Mental Disorders (ESEMeD) project. Acta Psychiatr Scand Suppl 2004(420):8-20.

27. de Graaf R, ten Have M, Burger H, Buist-Bouwman M. Mental disorders and service use in the Netherlands. Results from the European Study of the Epidemiology of Mental Disorders (ESEMeD) In: Ustun T, Kessler R, eds. The WHO World Mental Health Surveys: Global Perspectives on the Epidemiology of Mental Disorders. New York: Cambridge University Press; 2008:388-405.

28. de Graaf R, Ten Have M, van Dorsselaer S. The Netherlands Mental Health Survey and Incidence Study-2 (NEMESIS-2): design and methods. Int J Methods Psychiatr Res Sep 2010;19(3):125-141.

29. de Graaf R, ten Have M, van Gool C, van Dorsselaer S. Prevalence of mental disorders and trends from 1996 to 2009. Results from the Netherlands Mental Health Survey and Incidence Study-2. Soc Psychiatry Psychiatr Epidemiol Feb 2012;47(2):203-213.

30. Nuyen J, Tuithof M, de Graaf R, van Dorsselaer S, Kleinjan M, ten Have M. The bidirectional relationship between loneliness and common mental disorders in adults: findings from a longitudinal population-based cohort study. Social psychiatry and psychiatric epidemiology 2019:114.

31. de Graaf R, van Dorsselaer S, Tuithof M, ten Have M. Sociodemographic and psychiatric predictors of attrition in the third follow-up of the Netherlands Mental Health Survey and Incidence Study-2 (NEMESIS2). Utrecht: Trimbos Institute; 2018.

32. Stewart AL, Ware JE. Measuring functioning and well-being: the medical outcomes study approach: duke university Press; 1992.

33. HÅvard LJ, Kaasa S. Short form 36 (SF-36) health survey: normative data from the general Norwegian population. Scandinavian journal of social medicine 1998;26(4):250-258.

34. Fayaz A, Croft P, Langford RM, Donaldson LJ, Jones GT. Prevalence of chronic pain in the UK: a systematic review and meta-analysis of population studies. BMJ Open Jun 20 2016;6(6):e010364.

35. Vos T, Flaxman AD, Naghavi M, et al. Years lived with disability (YLDs) for 1160 sequelae of 289 diseases and injuries 1990-2010: a systematic analysis for the Global Burden of Disease Study 2010. Lancet Dec 15 2012;380(9859):2163-2196. 
36. Tegethoff M, Belardi A, Stalujanis E, Meinlschmidt G. Comorbidity of Mental Disorders and Chronic Pain: Chronology of Onset in Adolescents of a National Representative Cohort. The journal of pain: official journal of the American Pain Society Oct 2015;16(10):1054-1064.

37. Adler NE, Epel ES, Castellazzo G, Ickovics JR. Relationship of subjective and objective social status with psychological and physiological functioning: preliminary data in healthy white women. Health Psychol Nov 2000;19(6):586-592.

38. Radhakrishnan R, Guloksuz S, Ten Have M, et al. Interaction between environmental and familial affective risk impacts psychosis admixture in states of affective dysregulation. Psychol Med Aug 2019;49(11):1879-1889.

39. Pries LK, Guloksuz S, Ten Have M, et al. Evidence That Environmental and Familial Risks for Psychosis Additively Impact a Multidimensional Subthreshold Psychosis Syndrome. Schizophr Bull Jun 6 2018;44(4):710-719.

40. Brugha T, Bebbington P, Tennant C, Hurry J. The List of Threatening Experiences: a subset of 12 life event categories with considerable long-term contextual threat. Psychol Med Feb 1985:15(1):189194.

41. Schizophrenia Working Group of the Psychiatric Genomics Consortium. Biological insights from 108 schizophrenia-associated genetic loci. Nature Jul 24 2014;511(7510):421-427.

42. Pries LK, Klingenberg $B$, Menne-Lothmann $C$, et al. Interaction between polygenic liability for schizophrenia and childhood adversity influences daily-life emotional dysregulation and subtle psychosis expression submitted manuscript 2020.

43. Stahl EA, Breen G, Forstner AJ, et al. Genome-wide association study identifies 30 loci associated with bipolar disorder. Nat Genet May 2019:51(5):793-803.

44. Lee JJ, Wedow R, Okbay A, et al. Gene discovery and polygenic prediction from a genomewide association study of educational attainment in 1.1 million individuals. Nat Genet Jul 23 2018;50(8):1112-1121.

45. Savage JE, Jansen PR, Stringer S, et al. Genome-wide association meta-analysis in 269,867 individuals identifies new genetic and functional links to intelligence. Nat Genet Jul 2018;50(7):912919.

46. StataCorp. STATA Statistical Software: Release 16. Texas: College Station; 2019

47. Van Os J, Sham P. Gene-environment interactions. In: Murray RM, Jones PB, Susser E, Van Os J, Cannon M, eds. The Epidemiology of Schizophrenia. Cambridge: Cambridge University Press: 2003:235-254.

48. Vigod SN, Fung K, Amartey A, et al. Maternal schizophrenia and adverse birth outcomes: what mediates the risk? Social psychiatry and psychiatric epidemiology Dec 62019

49. Vigod SN, Kurdyak PA, Dennis CL, et al. Maternal and newborn outcomes among women with schizophrenia: a retrospective population-based cohort study. BJOG Apr 2014;121(5):566-574

50. Zhong QY, Gelaye B, Fricchione GL, Avillach P, Karlson EW, Williams MA. Adverse obstetric and neonatal outcomes complicated by psychosis among pregnant women in the United States. BMC Pregnancy Childbirth May 2 2018;18(1):120.

51. Gantriis DL, Thorup AAE, Harder S, et al. Home visits in the Danish High Risk and Resilience Study VIA 7: assessment of the home environment of 508 7-year-old children born to parents diagnosed with schizophrenia or bipolar disorder. Acta psychiatrica Scandinavica Aug 2019;140(2):126-134.

52. Simoila L, Isometsa E, Gissler M, Suvisaari J, Sailas E, Halmesmaki E, Lindberg N. Maternal schizophrenia and out-of-home placements of offspring: A national follow-up study among Finnish women born 1965-1980 and their children. Psychiatry research Mar 2019;273:9-14. 
53. de Sousa P, Varese F, Sellwood W, Bentall RP. Parental communication and psychosis: a metaanalysis. Schizophrenia bulletin Jul 2014;40(4):756-768.

54. Ranning A, Laursen T, Agerbo E, Thorup A, Hjorthoj C, Jepsen JRM, Nordentoft M. School performance from primary education in the adolescent offspring of parents with schizophrenia and bipolar disorder- a national, register-based study. Psychological medicine Sep 2018;48(12):19932000.

55. Kallquist A, Salzmann-Erikson M. Experiences of Having a Parent with Serious Mental IIIness: An Interpretive Meta-Synthesis of Qualitative Literature. Journal of Child and Family Studies 2019;28:2056-2068.

56. Agerbo E, Sullivan PF, Vilhjalmsson BJ, et al. Polygenic Risk Score, Parental Socioeconomic Status, Family History of Psychiatric Disorders, and the Risk for Schizophrenia: A Danish Population-Based Study and Meta-analysis. JAMA psychiatry Jul 2015;72(7):635-641.

57. Lund C, Brooke-Sumner C, Baingana $F$, et al. Social determinants of mental disorders and the Sustainable Development Goals: a systematic review of reviews. Lancet Psychiatry Apr 2018;5(4):357-369.

58. Forsman AK, Wahlbeck K, Aaro LE, et al. Research priorities for public mental health in Europe: recommendations of the ROAMER project. Eur J Public Health Apr 2015;25(2):249-254.

59. Kerlinger FN, Pedhazur EJ. Multiple regression in behavioral research. . New York: Holt, Rinehart and Winston; 1973. 



\section{CHAPTER 3}

\section{EVIDENCE THAT THE IMPACT OF CHILDHOOD TRAUMA ON IQ IS SUBSTANTIAL IN CONTROLS, MODERATE IN SIBLINGS AND ABSENT IN PATIENTS WITH PSYCHOTIC DISORDER}

\section{Published as:}

van Os J, Marsman A, van Dam D, Simons CJ; GROUP Investigators. Evidence That the Impact of Childhood Trauma on IQ Is Substantial in Controls, Moderate in Siblings, and Absent in Patients With Psychotic Disorder. Schizophr Bull. 2017 Mar 1;43(2):316-324. 


\section{ABSTRACT}

Research suggests childhood trauma is associated with cognitive alterations, but it is not known whether the cognitive alterations observed in patients with psychotic disorder, and their relatives, is trauma-related. Patients with a schizophrenia-spectrum diagnosis ( $n=1119)$, siblings of patients $(n=1059)$ and healthy comparison subjects (HCS; $n=586$ ) were interviewed 3 times over a period of 6 years. Repeated measures of IQ were analyzed as a function of childhood trauma and group, controlling for confounders. There were significant differences in the impact of childhood trauma on IQ across the 3 groups. Exposure in HCS was associated with a nearly 5- point reduction in IQ $(-4.85 ; 95 \%$ confidence interval $[\mathrm{CI}]:-7.98--1.73, \mathrm{p}=.002)$, a lesser reduction in siblings $(-2.58 ; 95 \% \mathrm{Cl}:-4.69--.46, \mathrm{p}=.017)$ and no significant reduction in patients (-0.84; $95 \% \mathrm{Cl}:-2.78-1.10, \mathrm{p}=.398)$.

One-fourth of the sibling-control difference in IQ was reducible to childhood trauma, whereas for patients this was only 5\%. Over the 6-year follow-up, those with trauma exposure showed significantly less learning effects with repeated cognitive assessments $(b=1.36,95 \% \mathrm{Cl}: 0.80-1.92, \mathrm{p}<.001)$ than the non-exposed $(b=2.31$, $95 \%$ Cl: $1.92-2.71, p<.001 ; p$ interaction $=.001)$

Although childhood trauma impacts cognitive ability and learning in non-ill people at low and high genetic risk, its effect on the observed cognitive alterations in psychotic disorder may be minor. Twin and family studies on cognitive alterations in psychotic disorder need to take into account the differential impact of trauma on cognition across ill and non-ill, at risk groups. 


\section{INTRODUCTION}

Childhood abuse and neglect has been found to impact many aspects of (social) cognition $^{1-8}$; impairments that have been suggested to persist into adulthood. ${ }^{9-11}$

Both a history of childhood trauma and cognitive impairments are highly prevalent in patients with psychotic disorders.12,13 Cognitive alterations are highly relevant clinically, impacting on community functioning, which may be mediated particularly by alterations in social cognition. ${ }^{14}$ Although it has been suggested that childhood trauma may contribute to the neurocognitive impairments observed in patients with nonaffective psychotic disorders, findings have been inconsistent across cognitive domains, diagnostic, demographic and genotype subgroups, and relatively small samples. ${ }^{15-21}$ Sideli and colleagues 22 reported an association between trauma and cognition in the controls, but not in patients, suggestive of a floor effect in patients, whose cognition already is substantially lower compared to controls. No studies to date had access to measures of cognition over time, in order to study not only any association between childhood trauma and cross-sectional measures of cognition, but also between childhood trauma and change of cognition over time.

In the current study, we attempted to study the link between childhood trauma and cognition taking into account the following issues: (1) adequate sample size to study main effects and test for moderation by group; (2) focus on schizophrenia spectrum diagnosis; (3) inclusion of endophenotypic measures of cognition in siblings of patients, in order to tease apart effects of etiological and disease-related factors; and (4) repeated measures of the cognitive outcome over time.

We hypothesized (1) a main effect of childhood trauma on cross-sectional cognitive outcome, conform the findings by Sideli and colleagues, 22 and (2) moderation by group (healthy comparison subjects [HCS], patients, siblings of patients). In addition, we hypothesized that those with exposure to childhood trauma would show less prominent learning effects with repeated cognitive assessments over time. Given evidence that (1) most of the overall effect of a schizophrenia diagnosis on cognitive performance is mediated through a single common factor, indicating that a generalized cognitive deficit is a core underlying feature, ${ }^{23}$ and (2) the effect of trauma on cognitive function is driven by deficits in general cognitive functioning ${ }^{17}$ the analyses focused on IQ as a summary measure of cognitive performance. 


\section{METHODS}

\section{GROUP study}

Full details of the GROUP study have been presented elsewhere. ${ }^{24,25}$ In representative geographical areas in the Netherlands and Belgium, patients were identified through clinicians working in regional psychotic disorder services, whose caseload was screened for inclusion criteria. Subsequently, a group of patients presenting at these services either as out-patients or in-patients were recruited for the study. HCSs were selected through random mailings to addresses in the catchment areas of the cases. The GROUP study was not conducted in a geographically well-defined small area, as it in fact included the majority of mental health services in the Netherlands, and a substantial part of mental health services in Dutch-speaking Belgium. HCSs could not be representative in all aspects, as an exclusion criterion was absence of a family history of psychotic disorder. The goal was to collect a group of HCSs that (1) was collected from the same geographical area as the case in the relevant mental health service, (2) was sufficiently large to allow for chance variation, (3) was frequency-matched in age- and sex distribution to the siblings, and (4) had absence of family history of psychotic disorder.

\section{Sample}

The full GROUP sample at baseline consisted of 1119 patients with non-affective psychotic disorder, 1059 siblings of these patients, 920 parents of the patients, and 586 unrelated HCSs. Inclusion criteria were: (1) age range 16 to 50 years and (2) good command of Dutch language. For patients, an additional inclusion criterion was the presence of a clinical diagnosis of non-affective psychotic disorder. HCSs status was confirmed by using the Family Interview for Genetic studies ${ }^{26}$ with the HCS as informant, to establish absence of first degree relatives with a psychotic disorder. Diagnosis was based on the Diagnostic and Statistical Manual of Mental Disorder-IV (DSM-IV) criteria, ${ }^{27}$ assessed with the Comprehensive Assessment of Symptoms and History (CASH) interview ${ }^{28}$ or Schedules for Clinical Assessment for Neuropsychiatry (SCAN 2.1). ${ }^{29}$ The majority of patients had a DSM-IV diagnosis of schizophrenia (DSM-IV 295.x; $n=940,84 \%$ ). In the sibling and healthy comparison subject groups, there were respectively 154 (14\%) and 59 participants (10\%) with a history of a common mental disorder at baseline, the majority of whom had a mood disorder (DSM-IV 296.x).

The study was approved by the standing ethics committee, and all the subjects gave written informed consent in accordance with the committee's guidelines. 
Follow-up

Patients, HCSs, and siblings were eligible for follow-up. Of the 586 HCSs and 1059 siblings at baseline, 78\% ( $n=1275$ ) were assessed at 3-year follow-up (healthy comparison subjects: $79 \%, n=460$; siblings: $77 \%, n=815)$ and $67 \%(n=1104)$ at six-year follow-up (HCSs: $67 \%, n=394$; siblings: $67 \%, n=710$ ). Ratings of CASH, SCAN and Community Assessment of Psychic Experiences (CAPE) ${ }^{30}$ at follow-up reflected the period between baseline and first follow-up, and between first and second follow-up, respectively. Mean time to first follow-up was 3.3 years $(S D=0.4)$ and mean between first and second follow-up was 3.1 years $(S D=0.4)$.

\section{Measures}

CAPE

The Community Assessment of Psychic Experiences (CAPE) was developed in order to rate self-reports of lifetime psychotic experiences. ${ }^{30}$ Items are modelled on patient experiences as contained in the PSE-931, schedules assessing negative symptoms such as the Scale for the Assessment of Negative Symptoms (SANS) ${ }^{32}$ and the Subjective Experience of Negative Symptoms (SENS) ${ }^{33}$, and scales assessing depressive symptoms such as the Calgary Depression Scale ${ }^{34}$. Items are scored on a 4-point scale. In the current analyses, CAPE dimensions of frequency of positive experiences (20 items), negative experiences (14 items) and depressive experiences ( 8 items) were included (measured at baseline and 3-year follow-up), representing the person's perceived psychosis load over the lifetime (at baseline) or in the past 3 years (follow-up). A total score representing the mean of all items was calculated for each dimension.

$1 Q$

At baseline and 3-year follow-=up, IQ was estimated based on the 4-subtest version (Information, Block Design, Digit Symbol Coding and Arithmetic) ${ }^{35}$ of the Wechsler Adult Intelligence Scale (WAIS-III). ${ }^{36}$ At 6-year follow-up, IQ was estimated based on a short version of the WAIS-III short form: the Digit Symbol Coding subtest, uneven items of the Arithmetic subtest, uneven items of the Block Design subtest, every third item of the Information subtest ${ }^{37}$. Change in IQ over the follow-up period (hereafter: delta IQ) was defined as change in IQ from baseline ( $\left.T_{0}\right)$ to $T_{2}$ at six years or, in case $T_{2}$ was missing, to $T_{1}$ at 3 years.

\section{Cannabis use}

Substance use was assessed repeatedly at baseline, 3-year follow-up (use over interval baseline and 3-year follow-up) and 6-year follow-up (use over interval 3-year follow-up and 6-year follow-up), using the Composite International Diagnostic Interview (CIDI). ${ }^{38} \mathrm{CIDI}$ cannabis pattern of use during the lifetime period of heaviest use (hereafter: cannabis 
frequency use) was used in the analyses as the exposure variable, in agreement with previous work in this sample, and was scored as: none (0), less than weekly (1), weekly (2), and daily (3) 39,40 , dichotomized in the analyses as 'no use' (0) versus 'any use' (1).

\section{Childhood trauma}

Childhood trauma was assessed with the Dutch version of the Childhood Trauma Questionnaire (CTQ) 25-item Short Form, ${ }^{41}$ with items rated on a 5-point Likert scale (1= never true to 5 = very often true). Emotional, physical, and general abuse, and emotional and physical neglect were assessed, 5 items covering each trauma type. ${ }^{41}$ The total trauma score represents the mean score of all 25 items. Childhood trauma was analyzed both as a continuous variable and as a dichotomous variable, around a cut-off representing the control group 80th percentile, conform previous analyses in this sample.42

\section{Statistical analyses}

GROUP database version 5.0 was used for all analyses. In order to examine crosssectional associations between childhood trauma and $I Q$, and differences herein as a function of group (controls, siblings, patients), random intercept multilevel regression models (given clustering of individuals within families as well as clustering of repeated measures within subjects) with IQ as dependent variable were fitted using the MIXED routine in the Stata program, version $14 .{ }^{43}$ Independent variables were CTQ score, group and the interaction between CTQ and group. Analyses were corrected a priori for age, sex, ethnic group (white European versus other), educational level (continuous variable ranging from 0 [no education], 3-5 [school diploma] to 8 [university degree]), CAPE total score and binary cannabis use (hereafter: fully adjusted model).

In order to examine associations between childhood trauma and change in IQ, and differences in this association across group, random intercept multilevel regression models with IQ as dependent variable were fitted with CTQ, time (baseline, 3-year and 6-year follow-up), group and the CTQ $x$ time $x$ group interaction. Analyses were corrected a priori for age, sex, ethnic group, educational level, CAPE total score and binary cannabis use (hereafter: fully adjusted model).

Interactions between childhood trauma and group were fitted in the models of IQ and delta $I Q$, yielding 2 interaction terms (1 for siblings and 1 for patients); in the case of significant interaction, stratified values for HCSs, siblings and patients were calculated from the model containing the interaction using the Stata MARGINS routine. Marginal effects and post-estimation contrasts were similarly calculated with the Stata MARGINS routine. Associations are expressed as regression coefficients ( $b$; change in $y$ with one unit increase in $x$ ) from the multilevel random regression model. 


\section{RESULTS}

\section{Sample characteristics}

Sample characteristics are displayed in table 1. The 3 groups were comparable in age, patients more often were of male sex. As reported before, siblings had values that were intermediate to HCSs and patients with regard to childhood trauma, ${ }^{44}$ cannabis use, ${ }^{45}$ and IQ. ${ }^{46}$ All groups displayed increases in IQ over the 6-year followup.

\section{Childhood trauma and IQ}

Table 2 displays IQ values at the 3 time points as a function of binary trauma exposure. Plotting IQ and continuous childhood trauma in the 3 groups suggested a negative association in HCSs, a weaker negative association in siblings and no associations in patients (figure 1). This was confirmed in the multilevel random regression analyses, which revealed a significant interaction between continuous childhood trauma and group in the model of $I Q$ (unadjusted: siblings $p=.027$, patients $\mathrm{p}$ <.001; adjusted for age, sex, education and ethnic group: siblings $p=$ .007, patients $\mathrm{p}<.001$; adjusted for age, sex, education, ethnic group, CAPE total score and cannabis use: siblings $p=.005$, patients $p<.001$ ). The association between $\mathrm{IQ}$ and childhood trauma, stratified by group, calculated from the fully adjusted model was -8.09 (95\% Cl: -11.50 - -4.68, p <.001; HCSs) -2.27 (-4.57 - .02, p = .052; siblings) and $-.18(-2.17-1.82, p=.863$; patients). Using the binary trauma variable in the fully adjusted model, stratified effect sizes for trauma exposure across the three groups were: -4.85 for HCSs (95\% Cl: $-7.98--1.73, \mathrm{p}<.001) ;-2.58$ for siblings (95\% Cl: $-4.69--.46, p=.017)$ and -.84 for patients (95\% Cl: $-2.78-1.10, p=.398)$.

In the fully adjusted model, the association between group and IQ, before entering continuous childhood trauma in the model, was -3.07 for siblings ( $95 \% \mathrm{Cl}$ : -4.57 - -1.57) and -9.66 for patients (95\% Cl: -11.24 - -8.07); after adding continuous childhood trauma to the model, this became -2.33 for siblings ( $95 \% \mathrm{Cl}$ : $-3.97--.69$ ) and -9.15 for patients (95\% Cl: $-10.93-7.38$ ). Thus, in siblings, $24 \%$ of the siblingHCSs difference in IQ was thus reducible to childhood trauma, whereas for patients this was $5 \%$. 
Table 1. Sample demographics and measures of childhood trauma and IQ at baseline (with the exception of delta IQ)

\begin{tabular}{lllllll}
\hline & & Age & Education $^{\mathbf{a}}$ & CTQ (continuous) & Urbanicity birth $^{\mathbf{b}}$ & IQ \\
\hline HCS & Mean & 30.42 & 2.92 & 1.34 & 2.64 & 109.82 \\
& SD & 10.58 & 1.28 & 0.36 & 1.69 & 15.00 \\
& $\mathrm{~N}$ & 584 & 583 & 492 & 549 & 573 \\
\hline Sibling & Mean & 27.83 & 2.56 & 1.41 & 2.66 & 102.87 \\
& SD & 8.27 & 1.47 & 0.41 & 1.67 & 15.49 \\
& N & 1059 & 1039 & 816 & 969 & 1012 \\
\hline Patient & Mean & 27.57 & 1.95 & 1.61 & 2.76 & 96.08 \\
& SD & 7.95 & 1.36 & 0.50 & 1.68 & 15.32 \\
& N & 1107 & 1086 & 755 & 981 & 1006 \\
\hline Total & Mean & 28.28 & 2.40 & 1.47 & 2.70 & 101.77 \\
& SD & 8.76 & 1.44 & 0.45 & 1.68 & 16.18 \\
& $\mathrm{~N}$ & 2750 & 2708 & 2063 & 2499 & 2591 \\
\hline
\end{tabular}

Note. CTQ: childhood trauma questionnaire; HCS: healthy comparison subjects.

a Education (Verhage): range 0 (no education), 3-5 (school diploma) to 8 (university degree).

b Urbanicity: $1 \leq 500 / \mathrm{km}^{2} ; 2=500-1000 / \mathrm{km}^{2} ; 3=1000-1500 / \mathrm{km}^{2} ; 4=1500-2500 / \mathrm{km}^{2} ; 5=2500+/ \mathrm{km}^{2}$

c Defined as change from baseline $\left(T_{0}\right)$ to $T_{2}$ at 6 years or, in case $T_{2}$ was missing, to $T_{1}$ at 3 years. 


\begin{tabular}{|c|c|c|c|c|}
\hline Delta IQ ${ }^{c}$ & \% CTQ binary exposure ${ }^{d}$ & $\%$ Cannabis use ${ }^{e}$ & $\%$ Female & \% Ethnic minority \\
\hline 4.32 & 0.19 & 0.28 & 0.54 & 0.10 \\
\hline \multicolumn{5}{|l|}{10.08} \\
\hline 428 & 492 & 579 & 586 & 586 \\
\hline 6.44 & 0.25 & 0.38 & 0.54 & 0.17 \\
\hline \multicolumn{5}{|l|}{9.52} \\
\hline 761 & 816 & 1047 & 1059 & 1059 \\
\hline 3.72 & 0.44 & 0.63 & 0.24 & 0.23 \\
\hline \multicolumn{5}{|l|}{10.20} \\
\hline 708 & 755 & 1094 & 1119 & 1119 \\
\hline 4.95 & 0.31 & 0.46 & 0.42 & 0.18 \\
\hline \multicolumn{5}{|l|}{9.98} \\
\hline 1897 & 2063 & 2720 & 2764 & 2764 \\
\hline
\end{tabular}

d Exposure defined as CTQ value $>80^{\text {th }}$ percentile of control CTQ value.

e Defined as CIDI cannabis pattern of use during the lifetime period of heaviest use, dichotomized as 'no use' (0) versus 'any use' (1). 
Table 2. IQ as a function of trauma exposure at baseline, 3-year and 6-year follow-up

\begin{tabular}{|c|c|c|c|c|c|}
\hline No trauma exposure ${ }^{a}$ & & Baseline & $\begin{array}{l}\text { 3-year } \\
\text { follow-up }\end{array}$ & $\begin{array}{l}\text { 6-year } \\
\text { follow-up }\end{array}$ & Total \\
\hline \multirow[t]{3}{*}{ Healthy comparison subject } & Mean & 111.20 & 113.10 & 116.76 & 113.42 \\
\hline & SD & 14.87 & 15.89 & 16.89 & 15.95 \\
\hline & $\mathrm{N}$ & 395 & 222 & 284 & 901 \\
\hline \multirow[t]{3}{*}{ Sibling } & Mean & 105.44 & 109.17 & 113.92 & 109.14 \\
\hline & SD & 15.53 & 17.03 & 17.51 & 16.96 \\
\hline & N & 591 & 425 & 455 & 1,471 \\
\hline \multirow[t]{3}{*}{ Patient } & Mean & 97.47 & 99.76 & 102.96 & 99.84 \\
\hline & SD & 15.00 & 16.26 & 17.34 & 16.27 \\
\hline & $\mathrm{N}$ & 393 & 295 & 305 & 993 \\
\hline \multirow[t]{3}{*}{ Total } & Mean & 104.82 & 107.15 & 111.49 & 107.54 \\
\hline & SD & 16.06 & 17.32 & 18.17 & 17.31 \\
\hline & $\mathrm{N}$ & 1,379 & 942 & 1,044 & 3,365 \\
\hline \multicolumn{6}{|l|}{ Trauma exposure ${ }^{a}$} \\
\hline \multirow[t]{3}{*}{ Healthy comparison subject } & Mean & 105.52 & 105.30 & 106.85 & 105.88 \\
\hline & SD & 14.16 & 16.49 & 16.62 & 15.50 \\
\hline & $\mathrm{N}$ & 90 & 53 & 65 & 208 \\
\hline \multirow[t]{3}{*}{ Sibling } & Mean & 100.61 & 102.84 & 107.22 & 103.26 \\
\hline & $S D$ & 14.63 & 14.14 & 17.46 & 15.67 \\
\hline & $\mathrm{N}$ & 197 & 120 & 144 & 461 \\
\hline \multirow[t]{3}{*}{ Patient } & Mean & 96.84 & 97.96 & 99.98 & 98.06 \\
\hline & SD & 15.90 & 16.54 & 16.42 & 16.26 \\
\hline & $\mathrm{N}$ & 305 & 206 & 205 & 716 \\
\hline \multirow[t]{3}{*}{ Total } & Mean & 99.42 & 100.53 & 103.58 & 100.96 \\
\hline & SD & 15.52 & 16.04 & 17.15 & 16.25 \\
\hline & $N$ & 592 & 379 & 414 & 1,385 \\
\hline
\end{tabular}

Note. ${ }^{\text {a }}$ Defined dichotomously as CTQ value $>80^{\text {th }}$ percentile of control CTQ value. 


\section{Childhood trauma and delta IQ}

Table 3 shows delta IQ for the 3 groups as a function of binary childhood trauma exposure. The results of the fully adjusted model with the three-way interaction between time, binary childhood trauma and group revealed a marginal effect of time (linear effect $b=2.03,95 \% \mathrm{Cl}: 1.67-2.39, \mathrm{p}<.001$ ) which differed as a function of binary childhood trauma ( $p$ interaction=0.0013), exposed individuals showing less of an increase in IQ over time ( $b=1.36,95 \% \mathrm{Cl}$ : .80 - 1.92, $\mathrm{p}<.001)$ than the non-exposed ( $b=2.31,95 \% \mathrm{Cl}: 1.92-2.71, \mathrm{p}<.001)$. The 3-way interaction between time, childhood trauma and group was not significant (siblings: $p=.393$; patients: $p=.820$ ), indicating that the moderating effect of childhood trauma on the effect of time on IQ did not differ between healthy comparison subjects, siblings and patients.

Table 3. Change in IQ (positive value indicates increase over time), defined as change from baseline $\left(T_{0}\right)$ to $T_{2}$ at 6 years or, in case $T_{2}$ was missing, to $T_{1}$ at 3 years, as a function of group and trauma exposure

\begin{tabular}{llll}
\hline & Mean delta IQ & SD & N \\
\hline No trauma exposure & & & \\
\hline Healthy comparison subject & 4.76 & 10.33 & 335 \\
Sibling & 6.86 & 9.51 & 514 \\
Patient & 4.34 & 10.19 & 344 \\
Total & 5.54 & 10.00 & 1193 \\
\hline Trauma exposure & & & 69 \\
\hline Healthy comparison subject & 2.59 & 9.00 & 161 \\
Sibling & 5.09 & 9.71 & 245 \\
Patient & 2.33 & 9.51 & 475 \\
\hline Total & 3.31 & 9.57 & \\
\hline
\end{tabular}




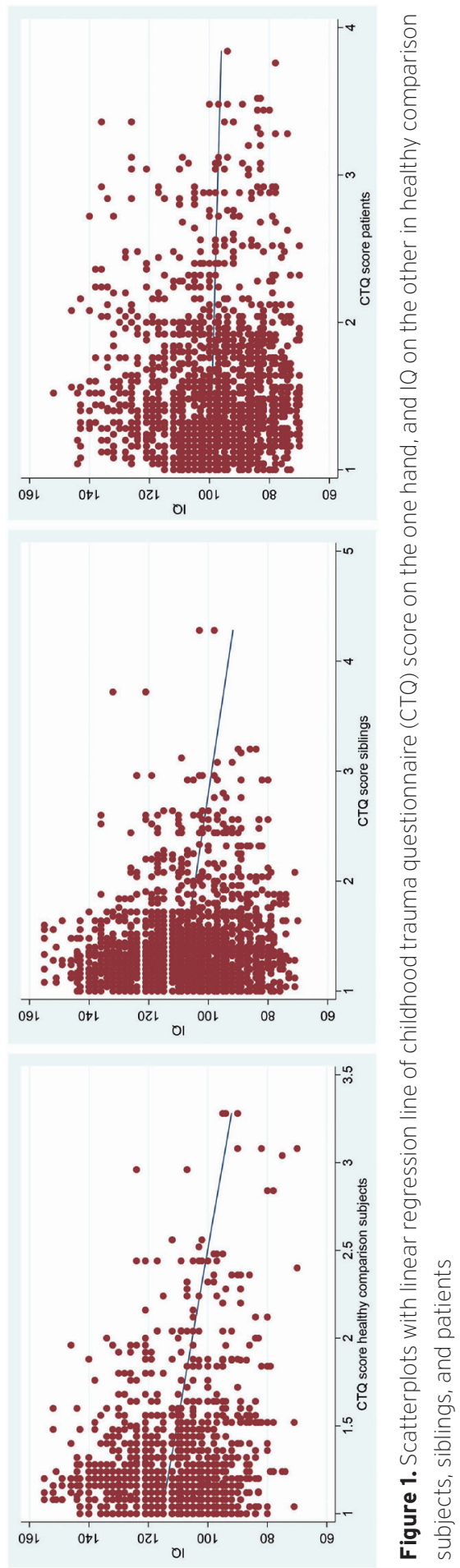




\section{DISCUSSION}

We found that childhood trauma impacted IQ in groups whose cognitive ability was not already compromised (HCSs) or only partially compromised (siblings of patients). Around a fourth of the sibling-healthy comparison subject difference was attributable to childhood trauma, compared to only $5 \%$ of case-healthy comparison difference. In addition, childhood trauma impacted on the course of IQ over time, in the sense of showing less learning effects of repeated cognitive assessments over time, regardless of group. The results suggest that childhood trauma impacts cognitive abilities and impedes learning; however, its impact on the observed cognitive alterations in psychotic disorder may be relatively small given smaller impact of childhood trauma on cognitive ability with progressively greater genetic risk for psychotic disorder.

Previous work indicates that the cognitive alterations observed in psychotic disorder are attributable to genetic liability underlying psychotic outcomes. ${ }^{47}$ However, the findings suggest that the observation of familial clustering of cognitive alterations in patients with psychotic disorder and their siblings is confounded by the fact that the source of these alterations is different across groups. In siblings, 24\% of the alterations of cognition respective to the healthy comparison group was reducible to childhood trauma, whereas in patients this was only $5 \%$. This suggests that sibling and twin studies on cognition of schizophrenia focusing on genetic factors need to take into account the influence of childhood trauma for accurate results.

Given the large sample size, diagnostic homogeneity, adjustment for confounders and the availability of repeated cognitive assessments over time, it is unlikely that the findings are due to chance or confounding. Several previous studies reported absence of association between childhood trauma main effects and cognitive outcomes in patients ${ }^{16,19,21,22}$ and there is a body of work showing association between childhood trauma and cognitive outcome in non-ill groups.1-11 Sideli and colleagues ${ }^{22}$ reported findings that resembled the current report, in that an association between childhood trauma and cognitive outcomes was found in the control, but not the patient group. One likely explanation for the absence of an association between trauma and cognition in the patient group is a floor effect - i.e., the impact of trauma is 'trumped' by other sources that impact on cognitive alterations, for example genetic effects, ${ }^{47}$ the effects of current adversity, ${ }^{48}$ or altered motivation to engage in neuropsychological testing. ${ }^{49}$ Although childhood trauma did not show main effects, it can still interact with any of these factors to impact cognition; future work is required to investigate this issue. The fact that childhood trauma impacted learning, regardless of group, does suggest that childhood trauma is not cognition-neutral in the patient group. 
There is evidence that, contrary to the current findings on IQ - considered an indicator of altered neurodevelopment in psychotic disorder 50 - that psychopathology, particularly psychotic symptoms, is associated with childhood trauma in both patients and individuals in the general population.12,51 Taken together, the results suggest that different etiological influences may impact different psychopathological and neurodevelopmental domains in psychotic disorder. The focus of treatment related to exposure to childhood trauma may be more in the realm of treating the psychological effects of trauma rather than with cognitive remediation of trauma-related cognitive alterations.52-54

Childhood trauma was associated with a decrease in the learning effect associated with repeated cognitive assessments. This impact did not differ between the different groups, including the patient group, suggesting that exposure to childhood trauma may have clinical relevance by moderating learning outcomes in patients with psychotic disorder. Although the effect was small, it may nevertheless be relevant in settings of rehabilitation and the application of cognitive remediation therapy.

\section{Methodological Issues}

Although the IQ assessment at second follow-up differed slightly, this cannot have impacted differential impact of childhood trauma across groups. Illness duration may be a factor in the patient group, potentially obscuring associations with childhood trauma. However, when modelling $I Q$ in the patient group as a function of childhood trauma and the interaction with illness duration (years between baseline assessment and onset of first psychotic symptom), no interaction was present ( $p=.110$ ), suggesting illness duration is not relevant for the association between IQ and childhood trauma. This finding is in agreement with the literature showing cognitive alterations in psychotic disorder are stable and not progressive..$^{55}$

\section{Funding}

The infrastructure for the GROUP study is funded through the Geestkracht programme of the Dutch Health Research Council (ZON-MW, grant number 10-000-1001), and matching funds from participating industry (Lundbeck, AstraZeneca, Eli Lilly, Janssen Cilag), universities and mental health care organizations (Amsterdam: Academic Psychiatric Centre of the Academic Medical Center and the mental health institutions: GGZ Ingeest, Arkin, Dijk en Duin, GGZ Rivierduinen, Erasmus Medical Centre, GGZ Noord Holland Noord. Maastricht: Maastricht University Medical Centre and the mental health institutions: GGZ Eindhoven en De Kempen, GGZ Breburg, GGZ Oost-Brabant, Vincent van Gogh voor Geestelijke Gezondheid, Mondriaan, Zuyderland, MetGGZ, RiaggVirenze Maastricht, Universitair Centrum Sint-Jozef Kortenberg, CAPRI University of Antwerp, PC Ziekeren Sint-Truiden, PZ Sancta Maria Sint-Truiden, GGZ Overpelt, 
OPZ Rekem. Groningen: University Medical Center Groningen and the mental health institutions: Lentis, GGZ Friesland, GGZ Drenthe, Dimence, Mediant, GGNet Warnsveld, Yulius Dordrecht and Parnassia psycho-medical center The Hague. Utrecht: University Medical Center Utrecht and the mental health institutions Altrecht, GGZ Centraal, Riagg Amersfoort and Delta). This study was funded in part by the European Community's Seventh Framework Program under grant agreement No. HEALTH-F2-2009-241909 (Project EU-GEI).

\section{Acknowledgments}

We are grateful for the generosity, in terms of time and effort, shown by the patients, their families and the healthy comparison subjects, and for the continuing collaboration with all the researchers who make the GROUP project possible. Furthermore, we would like to thank all research personnel involved in the GROUP project, in particular: Joyce van Baaren, Erwin Veermans, Ger Driessen, Truda Driesen, Karin Pos, Erna van 't Hag, Jessica de Nijs, Atiqul Islam, Wendy Beuken and Debora Op 't Eijnde.

\section{Genetic Risk and Outcome of Psychosis (GROUP) Investigators}

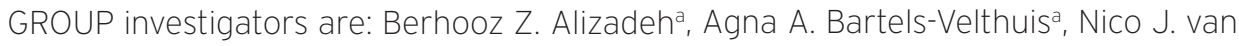
Beveren $^{b, c, d}$, Richard Bruggemanª, Wiepke Cahne, Lieuwe de Haanf, Philippe Delespaula, Carin J. Meijer ${ }^{\dagger}$, Inez Myin-Germeys ${ }^{h}$, Rene S. Kahne, Frederike Schirmbeck, Claudia J.P. Simons, ${ }^{g, i}$ Neeltje E. van Harene, Jim van Os ${ }^{g, j}$, Ruud van Winkel ${ }^{\text {g,h }}$

a University of Groningen, University Medical Center Groningen, University Center for Psychiatry, Groningen, The Netherlands; ' Antes Center for Mental Health Care, Rotterdam, The Netherlands; ' Erasmus MC, Department of Psychiatry, Rotterdam, The

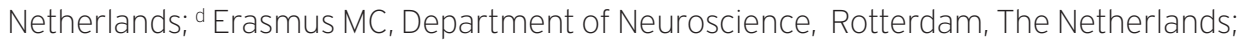
e University Medical Center Utrecht, Department of Psychiatry, Brain Center Rudolf Magnus, Utrecht, The Netherlands; ${ }^{\dagger}$ Academic Medical Center, University of Amsterdam, Department of Psychiatry, Amsterdam, The Netherlands; ${ }^{g}$ Maastricht University Medical Center, Department of Psychiatry and Psychology, School for Mental Health and Neuroscience, Maastricht, The Netherlands; ${ }^{h}$ KU Leuven, Department of Neuroscience, Research Group Psychiatry, Center for Contextual Psychiatry, Leuven, Belgium; ' GGzE, Institute for Mental Health Care Eindhoven and De Kempen, Eindhoven, The Netherlands; ' King's College London, King's Health Partners, Department of Psychosis Studies, Institute of Psychiatry, London, United Kingdom 


\section{REFERENCES}

1. Cicchetti D, Rogosch FA, Maughan A, Toth SL, Bruce J. False belief understanding in maltreated children. Dev Psychopathol Fall 2003;15(4):1067-1091.

2. Pears KC, Fisher PA. Emotion understanding and theory of mind among maltreated children in foster care: evidence of deficits. Dev Psychopathol Winter 2005;17(1):47-65.

3. Colvert E, Rutter M, Kreppner J, et al. Do theory of mind and executive function deficits underlie the adverse outcomes associated with profound early deprivation?: findings from the English and Romanian adoptees study. J Abnorm Child Psychol Oct 2008;36(7):1057-1068.

4. Fonagy P, Gergely G, Target $M$. The parent-infant dyad and the construction of the subjective self. J Child Psychol Psychiatry Mar-Apr 2007:48(3-4):288-328.

5. Hoffman-Plotkin D, Twentyman CT. A multimodal assessment of behavioral and cognitive deficits in abused and neglected preschoolers. Child Dev Jun 1984;55(3):794-802.

6. Perna RB, Kiefner M. Long-term cognitive sequelae: abused children without PTSD. Appl Neuropsychol Child 2013;2(1):1-5.

7. Bucker J, Kapczinski F, Post R, Cereser KM, Szobot C, Yatham LN, Kapczinski NS, Kauer-Sant'Anna M. Cognitive impairment in school-aged children with early trauma. Compr Psychiatry Aug 2012;53(6):758-764.

8. De Bellis MD, Hooper SR, Woolley DP, Shenk CE. Demographic, maltreatment, and neurobiological correlates of PTSD symptoms in children and adolescents. J Pediatr Psychol Jun 2010;35(5):570577.

9. Majer M, Nater UM, Lin JM, Capuron L, Reeves WC. Association of childhood trauma with cognitive function in healthy adults: a pilot study. BMC Neurol 2010;10:61.

10. Gould F, Clarke J, Heim C, Harvey PD, Majer M, Nemeroff CB. The effects of child abuse and neglect on cognitive functioning in adulthood. J Psychiatr Res Apr 2012;46(4):500-506.

11. Philip NS, Sweet LH, Tyrka AR, Carpenter SL, Albright SE, Price LH, Carpenter LL. Exposure to childhood trauma is associated with altered n-back activation and performance in healthy adults: implications for a commonly used working memory task. Brain Imaging Behav Mar 2016;10(1):124135 .

12. Varese F, Smeets F, Drukker M, et al. Childhood adversities increase the risk of psychosis: a metaanalysis of patient-control, prospective- and cross-sectional cohort studies. Schizophr Bull Jun 2012;38(4):661-671.

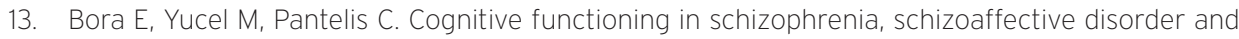
affective psychoses: meta-analytic study. Br J Psychiatry Dec 2009:195(6):475-482.

14. Fett AK, Viechtbauer W, Dominguez MD, Penn DL, van Os J, Krabbendam L. The relationship between neurocognition and social cognition with functional outcomes in schizophrenia: a metaanalysis. Neurosci Biobehav Rev Jan 2011;35(3):573-588.

15. Lysaker PH, Meyer P, Evans JD, Marks KA. Neurocognitive and symptom correlates of selfreported childhood sexual abuse in schizophrenia spectrum disorders. Ann Clin Psychiatry Jun 2001;13(2):89-92.

16. Aas M, Dazzan P, Fisher HL, et al. Childhood trauma and cognitive function in first-episode affective and non-affective psychosis. Schizophr Res Jun 2011;129(1):12-19.

17. Aas M, Steen NE, Agartz I, Aminoff SR, Lorentzen S, Sundet K, Andreassen OA, Melle I. Is cognitive impairment following early life stress in severe mental disorders based on specific or general cognitive functioning? Psychiatry Res Aug 15 2012;198(3):495-500. 
18. Shannon C, Douse K, McCusker C, Feeney L, Barrett S, Mulholland C. The association between childhood trauma and memory functioning in schizophrenia. Schizophr Bull May 2011;37(3):531-537.

19. McCabe KL, Maloney EA, Stain HJ, Loughland CM, Carr VJ. Relationship between childhood adversity and clinical and cognitive features in schizophrenia. J Psychiatr Res May 2012;46(5):600607.

20. Schenkel LS, Spaulding WD, DiLillo D, Silverstein SM. Histories of childhood maltreatment in schizophrenia: relationships with premorbid functioning, symptomatology, and cognitive deficits. Schizophr Res Jul 15 2005;76(2-3):273-286.

21. Green MJ, Chia TY, Cairns MJ, Wu J, Tooney PA, Scott RJ, Carr VJ, Australian Schizophrenia Research B. Catechol-O-methyltransferase (COMT) genotype moderates the effects of childhood trauma on cognition and symptoms in schizophrenia. J Psychiatr Res Feb 2014;49:43-50.

22. Sideli L, Fisher HL, Russo M, et al. Failure to find association between childhood abuse and cognition in first-episode psychosis patients. Eur Psychiatry Jan 2014;29(1):32-35.

23. Dickinson D, lannone VN, Wilk CM, Gold JM. General and specific cognitive deficits in schizophrenia. Biol Psychiatry Apr 15 2004;55(8):826-833.

24. Korver N, Quee PJ, Boos HB, Simons CJ, de Haan L. Genetic Risk and Outcome of Psychosis (GROUP), a multi site longitudinal cohort study focused on gene-environment interaction: objectives, sample characteristics, recruitment and assessment methods. Int J Methods Psychiatr Res Sep 2012;21(3):205-221.

25. G.R.O.U.P. Evidence that familial liability for psychosis is expressed as differential sensitivity to cannabis: an analysis of patient-sibling and sibling-control pairs. Arch Gen Psychiatry Feb 2011;68(2):138-147.

26. NIMH.Genetics.Initiative. Family Interview for Genetic Studies (FIGS). Rockville, Md: National Institute of Mental Health; 1992.

27. American Psychiatric Association. Diagnostic and statistical manual of mental disorders. 4th ed., text rev. ed: Washington, DC; 2000.

28. Andreasen NC, Flaum M, Arndt S. The Comprehensive Assessment of Symptoms and History (CASH). An instrument for assessing diagnosis and psychopathology. Arch Gen Psychiatry 1992:49(8):615-623.

29. Wing JK, Babor T, Brugha T, et al. SCAN. Schedules for Clinical Assessment in Neuropsychiatry. Arch Gen Psychiatry 1990;47(6):589-593.

30. Konings M, Bak M, Hanssen M, van Os J, Krabbendam L. Validity and reliability of the CAPE: a selfreport instrument for the measurement of psychotic experiences in the general population. Acta Psychiatr Scand Jul 2006;114(1):55-61.

31. Wing JK, Cooper JE, Sartorius N. The measurement and classification of psychiatric symptoms. London: Cambridge University Press; 1974.

32. Andreasen NC. Negative symptoms in schizophrenia. Definition and reliability. Arch Gen Psychiatry 1982;39(7):784-788.

33. Selten JP, Sijben NE, van den Bosch RJ, Omloo Visser J, Warmerdam H. The subjective experience of negative symptoms: a self-rating scale. Compr Psychiatry 1993;34(3):192-197.

34. Addington D, Addington J, Maticka-Tyndale E. Assessing depression in schizophrenia: the Calgary Depression Scale. Br J Psychiatry Suppl 1993(22):39-44.

35. Blyler CR, Gold JM, Iannone VN, Buchanan RW. Short form of the WAIS-III for use with patients with schizophrenia. Schizophr Res Dec 15 2000;46(2-3):209-215.

36. Wechsler D. WAIS-III: Wechsler Adult Intelligence Scale (3rd ed.) Administration and Scoring Manual. San Antonio, TX: Psychological Corporation; 1997. 
37. Velthorst $E$, Levine SZ, Henquet C, de Haan L, van Os J, Myin-Germeys I, Reichenberg A. To cut a short test even shorter: reliability and validity of a brief assessment of intellectual ability in schizophrenia--a control-case family study. Cogn Neuropsychiatry Nov 2013;18(6):574-593.

38. World Health Organisation. Composite International Diagnostic Interview (CIDI) Version 1.O. Geneva: World Health Organisation; 1990.

39. van Winkel R, Genetic Risk and Outcome of Psychosis (GROUP) Investigators. Family-based Analysis of Genetic Variation underlying Psychosis-Inducing Effects of Cannabis: Sibling Analysis and Proband Follow-Up. Archives of general psychiatry 2011;68(2):148-157.

40. van Winkel R, van Beveren JM, Genetic Risk and Outcome of Psychosis (GROUP) Investigators. AKT1 moderation of cannabis-induced cognitive alterations in psychotic disorder. Neuropsychopharmacology 2011;36(12):2529-2537.

41. Bernstein DP, Ahluvalia T, Pogge D, Handelsman L. Validity of the Childhood Trauma Questionnaire in an adolescent psychiatric population. J Am Acad Child Adolesc Psychiatry Mar 1997;36(3):340348.

42. van Nierop M, Janssens M, Genetic Risk and Outcome of Psychosis (GROUP) Investigators. Evidence that transition from health to psychotic disorder can be traced to semi-ubiquitous environmental effects operating against background genetic risk. PLoS One 2013;8(11):e76690.

43. StataCorp. STATA Statistical Software: Release 14. Texas: College Station; 2015.

44. Heins M, Simons C, Lataster T, et al. Childhood trauma and psychosis: a case-control and casesibling comparison across different levels of genetic liability, psychopathology, and type of trauma. Am J Psychiatry Dec 2011;168(12):1286-1294.

45. Genetic Risk and Outcome in Psychosis Investigators. Evidence that familial liability for psychosis is expressed as differential sensitivity to cannabis: an analysis of patient-sibling and sibling-control pairs. Arch Gen Psychiatry Feb 2011;68(2):138-147.

46. Verweij KH, Derks EM, Genetic Risk and Outcome of Psychosis (GROUP) Investigators. The association between intelligence scores and family history of psychiatric disorder in schizophrenia patients, their siblings and healthy controls. PLoS One 2013;8(10):e77215.

47. Toulopoulou T, Picchioni M, Rijsdijk F, Hua-Hall M, Ettinger U, Sham P, Murray R. Substantial genetic overlap between neurocognition and schizophrenia: genetic modeling in twin samples. Arch Gen Psychiatry Dec 2007;64(12):1348-1355.

48. Mani A, Mullainathan S, Shafir E, Zhao J. Poverty impedes cognitive function. Science Aug 30 2013;341(6149):976-980.

49. Fervaha G, Zakzanis KK, Foussias G, Graff-Guerrero A, Agid O, Remington G. Motivational deficits and cognitive test performance in schizophrenia. JAMA Psychiatry Sep 2014;71(9):1058-1065.

50. Jones P, Rodgers B, Murray R, Marmot M. Child development risk factors for adult schizophrenia in the British 1946 birth cohort. Lancet Nov 19 1994;344(8934):1398-1402.

51. van Dam DS, van Nierop M, Viechtbauer $W$, et al. Childhood abuse and neglect in relation to the presence and persistence of psychotic and depressive symptomatology. Psychol Med May 2015;45(7):1363-1377.

52. Mueser KT, Goodman LB, Trumbetta SL, Rosenberg SD, Osher C, Vidaver R, Auciello P, Foy DW. Trauma and posttraumatic stress disorder in severe mental illness. J Consult Clin Psychol Jun 1998;66(3):493-499.

53. Frueh BC, Grubaugh AL, Cusack KJ, Elhai JD. Disseminating evidence-based practices for adults with PTSD and severe mental illness in public-sector mental health agencies. Behav Modif Jan 2009;33(1):66-81. 
54. Frueh BC, Grubaugh AL, Cusack KJ, Kimble MO, Elhai JD, Knapp RG. Exposure-based cognitivebehavioral treatment of PTSD in adults with schizophrenia or schizoaffective disorder: a pilot study. J Anxiety Disord Jun 2009;23(5):665-675.

55. Zipursky RB, Reilly TJ, Murray RM. The myth of schizophrenia as a progressive brain disease. Schizophr Bull Nov 2013;39(6):1363-1372. 



\section{CHAPTER 4 \\ THE IMPACT OF ADVERSE CHILDHOOD EXPERIENCES ON EMG REACTIVITY: A PROOF-OF-CONCEPT STUDY}

Published as:

Marsman A, Luijcks R, Vossen C, van Os J, Lousberg R. The impact of adverse childhood experiences on EMG reactivity: A proof of concept study. PLoS One. 2019; 14(5). 


\section{ABSTRACT}

Adverse childhood experiences (ACE), such as emotional or physical abuse, can produce a lasting effect on the individual. The aim of this study was to investigate how ACE may impact electromyography (EMG) activity of the trapezius muscle in a novel experimental stress paradigm, in a sample of 120 healthy participants. The stress paradigm consisted of a memory task, in which participants were asked to memorize and recall as many words as possible, displayed on a screen. The study protocol included 2 identical experimental sessions ( $\mathrm{T}_{0}=0$ and $\mathrm{T}_{1}=6$ months). EMG activity was analyzed using multilevel regression analysis. EMG activity was higher during the memory task compared to baseline, supporting the validity of the experimental EMG-stress paradigm. In addition, the EMG increase was attenuated during the second session. Analyses were indicative for a moderating effect of ACE on stress- induced EMG activity: higher ACE scores resulted in greater EMG reactivity. These associations were apparent for early ACE exposure (0-11 years) as well as for later exposure (12-17 years). The association between ACE and EMG reactivity remained significant but was much weaker at $T_{1}$ in comparison to $T_{0}$, likely because of reduced unpredictability and uncertainty related to the experiment.

In conclusion, this study showed that enduring liabilities occasioned by ACE in a non-clinical population can be studied using an experimental paradigm of EMG stress reactivity, contingent on the level of predictability of the stressor. 


\section{INTRODUCTION}

There is extensive evidence that adverse childhood experiences (ACEs), such as emotional or physical abuse, can have long lasting effects on the individual. ${ }^{1-3}$ Several studies have revealed that ACEs negatively impact neurobiological processes, immunological parameters and autonomic, endocrine and metabolic systems. ${ }^{4-7}$ There is also evidence that exposure to early life adversity increases stress sensitivity later in life, which in turn is thought to increase vulnerability to develop mental disorders such as depression and psychosis following adult stressful life events. ${ }^{2,8}$ Additionally, in the Adverse Childhood Experiences Study (ACE Study) - a large epidemiological research project conducted in the United States to assessing the long-term effects of childhood abuse on adult health problems - a link between many types of childhood adversity and adult onset of somatic and mental health disorders was apparent. 910 In addition, there is evidence of a dose-response relationship in the association between ACEs and later mental health problems. ${ }^{9,1,12}$

The experience of early life adversity can be highly variable, for instance with regard to factors such as type of maltreatment and duration, predictability and severity of the stressor. Regardless of these factors however, ACEs by nature share the commonality of being stressful and stress inducing. 12

Although it may be hypothesized that ACEs considered as 'more severe' (e.g., sexual abuse as opposed to financial problems) result in more disadvantage, findings of the ACE Study showed that ten different types of adversity exerted equivalent negative effects. ${ }^{9}$ One explanation for this finding was offered by Dong and colleagues, who studied data from the larger ACE Study and demonstrated that ACEs often co-occur and should thus be viewed as a complex set of highly interrelated experiences, rather than stand-alone events. ${ }^{13}$ Thus, when assessing the impact of ACE, the authors suggest that the experiences should not be assumed to be isolated events during childhood but rather as co-occuring with other types of adversity. ${ }^{13}$

Another explanation for the apparent equivalence of negative effects pertaining to different ACEs is that the developing brain reacts similarly to different types and degrees of adversity because they share the common feature of unpredictability. ${ }^{12}$ During childhood, the unpredictability of an event (e.g., not knowing when, why or from where an emotional or physical event may arise) may be most impactful. Evidence suggests that unpredictability associated with ACE exposure may be particularly important for younger children. ${ }^{14}$ This is compatible with literature suggesting that ACE exposure in early childhood (0-11 years) is associated with more harmful effects than exposure at an older age (12-17 years) ${ }^{1,3}$ 
Animal research on chronic unpredictable stress supports the notion that it is particularly the degree of unpredictability of stress that mediates its harmful consequences. In one study, rats were exposed to unpredictable mild stressors for three weeks. ${ }^{15}$ During this time, at unpredictable moments, one of three stressors was applied: cage rotation, one-day food restriction or thirty minutes exposure to strobe lights. After three weeks, effects on the brain were evaluated. The group that was exposed to unpredictable mild stressors showed significant changes in hippocampal receptors - a brain area associated with emotion and stress regulation. The control group that experienced no stress showed no brain changes. ${ }^{15}$ The authors followed up this finding and found that when stress is completely predictable, even if more severe, brain changes were much less apparent compared to three weeks of moderate unpredictable stress. ${ }^{12}$

While many studies have demonstrated the long-lasting negative effects of adverse childhood experiences on future health and well-being, relatively little is known about etiological underlying (biopsychosocial) mechanisms. However, there is widespread consensus on two key aspects related to the impact of stress. First, the concept of 'stress' itself is best conceptualized as a theoretical construct that is not directly measurable. ${ }^{16}$ Second, stress per definition induces (psycho)physiological changes, such as in heart rate, cortisol levels and muscle tension levels, varying from hardly noticeable to extreme.17,18

In this context, Luijcks and coworkers showed that trapezius muscle stress reactivity is a good candidate for examining the impact of ACE on a physiological stress-related outcome. ${ }^{19-21}$ These authors examined the association between ACE and muscle activity in a recently developed experimental stress paradigm, in which the focus was on anticipatory cognitive stress preceding a single unpredictable and uncontrollable electrical painful stimulus. They found that anticipatory electromyography (EMG) stress reactivity during the experiment, indexed as trapezius muscle activity, was consistently stronger in participants with higher levels of ACE. It was furthermore shown that early childhood ACE (0-11 years) had a stronger moderating effect than adolescent ACE (1217 years). ${ }^{21}$ This finding was true for both session 1 at the beginning of the study and session 2 at 6 months, confirming the reliability of the experiment.

In the present study, we wish to extend the findings by Luijcks and colleagues on the influence of ACE on EMG activity to a different experimental stress paradigm. This paradigm consisted of an EMG baseline measurement followed by a novel memory task in which participants were instructed to memorize and recall as many words as possible, displayed on a screen. In contrast to the experimental stress experiment used by Luijcks and coworkers, which included a physical stressor (an electrical shock), in the 
current study, the stressor impacting trapezius muscle activity was cognitive by nature. Previous research has shown that cognitive tasks such as the Stroop color word test and mental arithmetic tasks successfully induce mental stress, resulting in changes in EMG reactivity of the trapezius muscle..$^{20,22}$ In the current paradigm we propose a newly designed memory task for stress induction that is easier to apply and generalizes better to daily life stress than for instance the Stroop test does.

In order to investigate the role of predictability in the experiment, the study protocol included 2 identical experimental sessions for each participant ( $T_{0}=0$ months, session 1 , and $T_{1}=6$ months, session 2). Since the experimental stress memory task had not been used before, validation of the experimental EMG-stress paradigm was the first objective of the study. To this end, two effects were tested, one main condition effect (baseline vs memory task) and one condition*session interaction effect. The a priori hypothesis was the existence of a significant positive condition effect (higher EMG reactivity during the memory task compared to baseline) as well as a negative condition*session effect (lower reactivity during session 2 compared to session 1). Furthermore, a significant main effect of age was expected, increasing age resulting in lower EMG activity. ${ }^{23}$ The following set of variables were added as potential confounders: NEO-neuroticism scale, STAI (state version), perceived stress (PSS), educational level and life events in the previous year. In the method section, these variables are explained in more detail.

The second objective was to further explore the association between ACE and stressrelated trapezius muscle activity. To this end, we investigated the cross-experiment generalizability of the finding by Luijcks and colleagues that ACE influences EMG stress reactivity in a cognitive stress experiment. Based on previous research, it was hypothesized that exposure to ACE, particularly those occurring during early childhood, would be associated with increased trapezius muscle activity during the memory task. Furthermore, it was hypothesized that the moderating effect of ACE would be smaller 6 months later, given a reduction in unpredictability during the second session (compared to the first session).

Since multilevel regression analysis was used to test the hypotheses, ${ }^{21}$ we were able to also include random effects. For all models, we hypothesized a random intercept (representing general EMG variability between participants), a random condition effect (indicating differential task reactivity) as well as a random session effect (demonstrating between-session variability). 


\section{METHODS}

\section{Ethics Statement}

The experiment was part of a larger study that evaluated psychophysiological reactivity as a predictor of change in pain and depressive complaints. The original study was conducted according to the principles of the Declaration of Helsinki and was approved by the medical ethics committee of the Academic Hospital Maastricht and Maastricht University (METC azM/UM, NL40284.068.12/METC 12-3-015). All participants provided written informed consent before the start of the experiment.

\section{Participants}

The sampling frame was a general population sample derived from the population living in the city of Maastricht, the Netherlands, who had responded to flyers that were handed out in several public places. One hundred and twenty participants (78 women and 42 men) participated in the study. Of these, 114 were right-handed. Age ranged from 18 to 65 years (mean 40.5, SD 17.1). Exclusion criteria were structural use of antipsychotics, antiepileptics or anxiolytics during the past year or structural use of alcohol in excess of $7 \mathrm{u}$ /day. Participants were asked to refrain from alcoholic beverages the evening before the experiment, and to refrain from caffeine-containing consumptions three hours prior to the experiment. Participants received $€ 50$ for participating in the study.

\section{Procedure}

Upon arrival in the laboratory, participants were first asked to complete a set of questionnaires, after which EMG and ECG electrodes were attached. EMG electrodes were attached on the left and right trapezius muscles (LTM and RTM). The baseline measurement was 5 minutes, in which participants were instructed to sit as still as possible, with their eyes open. After the baseline, participants continued with the memory task. Participants were instructed to look at a screen on which words appeared, and were asked to memorize as many words as possible. In a fixed (category) semirandomized order, 40 words were presented on the screen, each word displayed for 2 seconds. The 40 words were divided into 4 categories: positive connotation, negative connotation, pain-related and neutral. Each category contained 10 words. After the presentation of 40 words, participants had 1 minute to recall all memorized words. This procedure was repeated 3 times, and each time participants were asked to perform as best as they could. The study protocol included an identical follow-up measurement 6 months after the first session. 


\section{Psychophysiological recordings}

EMG activity was recorded from the left and right upper trapezius muscle. Recordings were conducted in an electrically and sound-shielded cubicle $\left(7.1 \mathrm{~m}^{2}\right)$, using $\mathrm{Ag} / \mathrm{AgCl}$ electrodes centered on a point $2 \mathrm{~cm}$ lateral to the midpoint between the acromion process and spinous process of the seventh cervical vertebra. A reference electrode was placed over the spinous process of the seventh cervical vertebra. ECG activity was recorded with a standard 3-lead ECG. Conductive paste (Ten20) was used to fix all electrodes. Brainvision BrainAmp Research Amplifier was used for all recordings. ECG and EMG were sampled with $1000 \mathrm{~Hz}$.

\section{Psychological measurements}

Adverse childhood experiences (ACE)

Assessed with a questionnaire developed within the context of the FP7 EU-GEI project (European Network of National Schizophrenia Networks Studying Gene-Environment Interactions). ${ }^{24}$ The Childhood Experiences of Care and Abuse questionnaire comprises 15 yes/no questions. Examples of items are: 'Were your basic needs ever neglected?' and: 'Was there an adult person you could talk to about problems or your feelings?' In addition to these items, issues such as the presence of financial problems in the family, the occurrence of sexual abuse, and so on, were assessed. The questionnaire covers two exposure periods: the first period includes exposure between 0 and 11 years (early childhood), the second period between 12 and 17 years (adolescence). Prior research has shown adequate internal consistency coefficients for both age periods. ${ }^{21}$ The maximum score in each age period was 15, the maximum score for the entire questionnaire was 30. The sum of scored events for both age periods together ranged from 0 to 14 (mean 3.4, SD 3.4). For the exposure period of 0 to 11 years, the sum of scored events ranged from 0 to 9 (mean 1.8, SD 1.9), whereas for the exposure period of 12 to 17 years, the sum of scored events ranged from 0 to 8 (mean 1.6, SD 1.8).

\section{Neuroticism}

Assessed with the Revised Neuroticism-Extraversion-Openness Personality Inventory (NEO-PI-R). The NEO-PI is a 240-item self-rating questionnaire that measures five major personality dimensions. Items are scored on a five-point Likert scale, ranging from 1 (disagree strongly) to 5 (agree strongly). The questionnaire has been validated extensively ${ }^{25}$ and is widely used to operationalize the five-factor model of personality. ${ }^{26}$ From the NEO-PI-R a total neuroticism score was calculated, representing a tendency or predisposition to experience negative affective states such as anxiety, depression, anger and impulsiveness. The total score ranged from 80 to 200 (mean 130, SD 22.5). 
State anxiety

Assessed with the State-Trait Anxiety Inventory (STAI), a commonly used measure of trait and state anxiety. ${ }^{27}$ The questionnaire consists of 20 items assessing trait anxiety and 20 for state anxiety. The State Anxiety scale assesses the current anxiety state using items such as 'I am tense; I am worried' and 'I feel calm; I feel secure'. Items are rated on a 4-point Likert scale ranging from 1 (almost never) to 4 (almost always). Higher scores indicate greater anxiety. The total score ranged from 20 to 77 (mean 35.7, SD 10.3).

\section{Perceived Stress}

Assessed by the Perceived Stress Scale (PSS), the most widely used instrument with acceptable psychometric properties for measuring the perception of stress in daily life. ${ }^{28}$ The PSS is a 10-item questionnaire, asking about feelings and thoughts during the last month. Items such as 'In the last month, how often have you been upset because of something that happened unexpectedly?' are rated on a 5-point Likert scale ranging from 0 (never) to 4 (very often). Total score ranged from 20 to 39 (mean 11.3, SD 6.2).

\section{Recent life stressors}

Assessed by asking participants whether or not they had experienced one or more events that impacted their daily lives in the previous year. A few examples of life events from the Holmes-Rahe Stress Inventory ${ }^{29}$ were provided to give participants an idea of what should be regarded a 'life event'. In order to keep the experiment as short as possible the assessment only consisted of a yes/no question, instead of using the entire Holmes-Rahe Stress Inventory. Overall, 86\% of the participants experienced at least one life event in the previous year.

\section{Offline data processing}

EMG data was filtered offline (low pass $0.5 \mathrm{~Hz}$, high pass $250 \mathrm{~Hz}, 50 \mathrm{~Hz}$ notch filter) and segmented into segments of 2000 milliseconds. Raw data were visually inspected for artifacts using software (BrainVision Analyzer 2.0) and excluded from further analyses when detected. EMG activity was corrected for ECG activity. Using regression analysis, the variance due to ECG activity was removed from the uncorrected EMG variable. Next, for each 2000 ms segment, the root mean square (RMS) value was calculated followed by a $10 \mathrm{log}$ logarithmic transformation to preserve a normal distribution. Due to hardware memory limitations, the number of total segments had to be restricted in order to perform the multilevel regression analysis. Consequently, for each participant, the 5 minutes baseline period was divided into 30 segments, each with a duration of 10 seconds. The memory task was also divided into 10-second segments (resulting in 24 segments), each segment containing 5 consecutive words. 


\section{Statistical analysis}

The EMG dataset had a hierarchical structure consisting of repeated time segments (level 1), nested within individuals (levels 2). Given this structure, multilevel regression analysis was performed, in which EMG activity of the LTM and RTM served as the dependent variable in all models.

As outlined above, we first executed a series of validation analyses. We tested a main condition effect contrasting baseline (coded ' $O$ ') with the memory task (coded ' 1 ') and a condition*session interaction effect (where session was coded ' $O$ ' at $T_{0}$ and ' 1 ' at $T_{1}$ ). Additionally, age (in years) and sex were included as predictor variables, as well as the NEO-neuroticism scale, STAI (state version), perceived stress (PSS), educational level and life events in the previous year.

In the next series of models, ACE score for the early childhood period and the adolescent period were included as key predictor variables, in separate models. The third-order interaction between ACE, session and condition was of main interest in these models. As argued in the introduction, we expected negative coefficients of this interaction term (representing a decreased moderating effect of ACE during the second session), particularly in the model including early childhood ACE.

Initially, we also planned to analyze the impact of the 4 different word categories (see above) on EMG reactivity. However, the multilevel analyses could not accommodate the extra number of records required for these analyses.

In order to test which covariance structure should be applied for this dataset, various covariance structures were tested. In agreement with earlier work with comparable experiments, ${ }^{21,30}$ AR1 yielded the best fit. All models were run with a random intercept, random condition and random session effect. All statistical analyses were performed using SPSS 24.0. P-values $\leq .05$ were considered to be statistically significant. 


\section{RESULTS}

A total of 120 participants were enrolled (78 female, 42 male). At the second experimental session, there was attrition of 15 participants. Thus, the multilevel dataset consisted of 120 participants for session 1 and 105 participants for session 2. General characteristics of the sample are displayed in table 1. Independent sample t-tests and Chi-square tests revealed that there was no significant difference between variables at the first and second measurement (all $p>$.653). Approximately 40\% of all participants experienced 2 or more adverse events.

Table 1. General characteristics and descriptive statistics of the study population

\begin{tabular}{|c|c|c|}
\hline Variables & Session $1(N=120)$ & Session $2(N=105)$ \\
\hline Age, mean (SD) min-max & $39.70(17.06) 18-66$ & 41.01 (16.99) 18-66 \\
\hline \multicolumn{3}{|l|}{ Gender (\%) } \\
\hline Male & 35.3 & 36.1 \\
\hline Female & 64.7 & 63.9 \\
\hline \multicolumn{3}{|l|}{ Educational level (\%) } \\
\hline Lower & 32.3 & 32.3 \\
\hline Middle & 26.1 & 26.8 \\
\hline Higher & 41.6 & 40.9 \\
\hline \multicolumn{3}{|l|}{ Life event(s) past year (\%) } \\
\hline No & 13.5 & 14.4 \\
\hline Yes & 86.5 & 85.6 \\
\hline \multicolumn{3}{|l|}{ Early ACE } \\
\hline None & 31.1 & 30.6 \\
\hline 1 & 24.4 & 24.7 \\
\hline 2 & 17.2 & 17.0 \\
\hline$>2$ & 27.3 & 27.7 \\
\hline \multicolumn{3}{|l|}{ Adolescent ACE } \\
\hline None & 34.5 & 34.6 \\
\hline 1 & 26.9 & 26.3 \\
\hline 2 & 13.4 & 15.4 \\
\hline$>2$ & 25.2 & 23.7 \\
\hline STAl-state, mean (SD) min-max & 35.89 (10.17) $20-77$ & 35.45 (10.39) $20-77$ \\
\hline PSS-score, mean (SD) min-max & $1.13(.61) .20-3.90$ & $1.12(.62) .20-3.90$ \\
\hline NEO-neuroticism, mean (SD) min-max & $129.86(22.04) 80-200$ & $130.10(23.06) 80-200$ \\
\hline
\end{tabular}

Note. ACE, Adverse Childhood Experience; STAI, State-Trait Anxiety Inventory, state-subscale; PSS, Perceived Stress Scale; NEO, Neuroticism-Extraversion-Openness Personality Inventory, neuroticism-subscale. 


\section{Validation analyses: EMG activity during baseline and memory task}

As hypothesized, a significant EMG increase was demonstrated during the memory task compared to baseline, for both left $(t=3.861, p<.001)$ and right $(t=4.788, p<.001)$ trapezius muscle. Contrary to our expectations, we did not observe a significant main association with age ( $p=.099)$. We did find a positive association between EMG and education level for both left ( $t=2.608, p=.011$ ) and right $(t=2.062, p=.042$ ) trapezius muscle activity, indicating that people with higher educational levels show increased EMG activity compared to people with lower educational levels.

With respect to the second validation hypothesis, we investigated whether there was a condition*session interaction, indicating lower EMG reactivity at session 2 compared to session 1.12 A significant interaction was found for both LTM $(t=-7.197, p<.001)$ and RTM ( $t=-5.763, p<.001)$. Post-hoc, it was investigated whether baseline level EMG differed between the two sessions, as suggested in figure 1. However, this was not the case, as indicated by a non-significant association with session for both LTM ( $p=.548$ ) and RTM ( $p=$.072). Results of significant associations related to EMG are displayed in table 2.As described in the method section, EMG data were 10log-transformed. In order to calculate the EMG condition effect in its original (untransformed) scale, a backtransformation was performed. In its original scale, EMG task reactivity was 2.1 times larger at $\mathrm{T}_{0}$ than at $\mathrm{T}_{1}$ for RTM and 3.59 times for LTM.

Finally, all models showed a significant random intercept, random condition and random session effect (all $p<.001$ ), indicating that participants differed regarding general (task irrelevant) EMG variability as well as task reactivity (condition effect) and additionally displayed between session variability.

Table 2. Significant associations with EMG of the trapezius muscle

\begin{tabular}{lllllll}
\hline & & $\boldsymbol{\beta}$ & $\mathbf{S E}$ & $\mathbf{d f}$ & $\mathbf{t}$ & \multicolumn{1}{c}{$\mathbf{p}$-value } \\
\hline EMG LTM & Task & .066 & .017 & 108.058 & 3.861 & $<.001$ \\
& Age & .079 & .037 & 100.129 & 2.145 & .034 \\
& Higher education & .131 & .050 & 98.839 & 2.608 & .011 \\
& Condition*session & -.072 & .010 & 2428.676 & -7.197 & $<.001$ \\
\multirow{2}{*}{ EMG RTM } & Task & .076 & .016 & 106.837 & 4.788 & $<.001$ \\
& Age & -.002 & .001 & 98.019 & -1.664 & .099 \\
& Higher education & .109 & .053 & 97.452 & 2.062 & .042 \\
& Condition*session & -.057 & .010 & 1874.213 & -5.763 & $<.001$ \\
\hline
\end{tabular}

Note. LTM, left trapezius muscle; RTM, right trapezius muscle; Task coded as 0 = baseline, $1=$ memory task. 


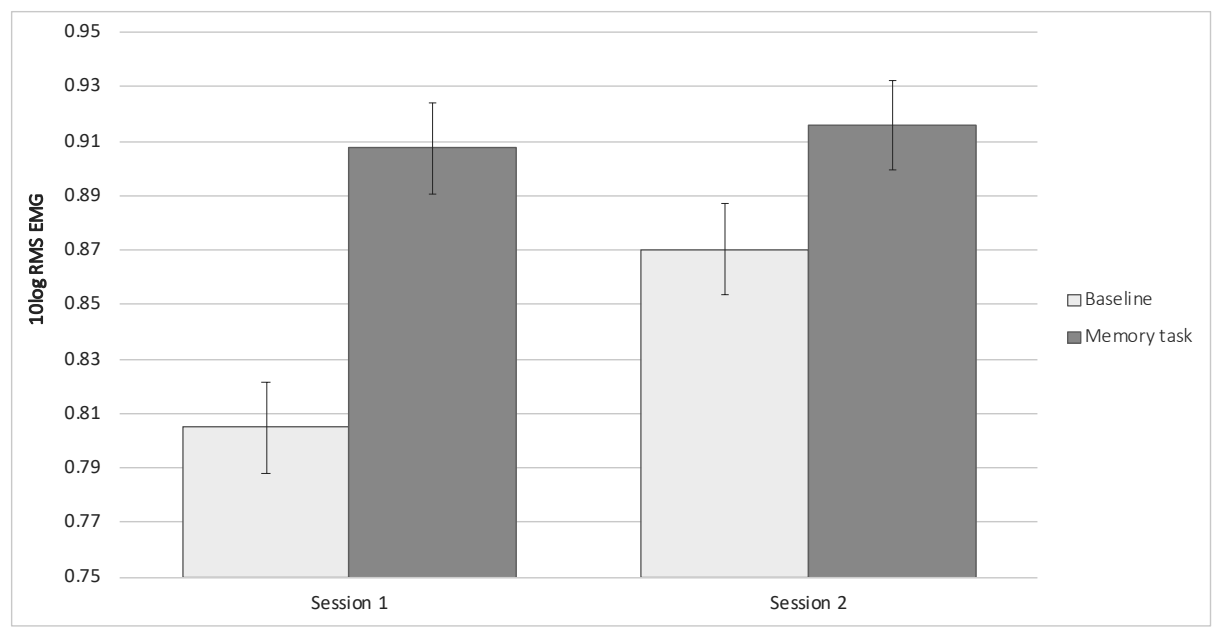

Figure 1. Predicted mean EMG (10log RMS) of the right trapezius muscle Note. Mean EMG was higher during the memory task at both sessions. Task-reactivity was greater at session 1 compared to session 2 (condition*session interaction significant $p<.001$ ).

\section{The interaction between ACE and EMG reactivity over time}

It was investigated whether the condition*session effect was moderated by ACE score. For each ACE score (early childhood and adolescence) we ran a third-order interaction model with a condition*session*ACE score interaction variable. All models were adjusted for the set of seven possible confounders as described in the method section.

The third-order interaction for LTM was significant for both the model with the early childhood ACE score $(\beta=-.018, t=-3.269, p=.001)$ and the adolescent ACE score ( $\beta=-.017, t=-2.965, p=.003$ ). For RTM no significant (all $p$-values $>$.711) third-order interactions were found. Post-hoc, we investigated whether excluding left-handed participants $(n=6)$ meaningfully changed the results of the analyses. This was not the case.

In order to further explore the meaning of the third-order interaction, each ACE score was dichotomized at the median split $(\mathrm{O}=$ relatively low ACE score, 1 = relatively high ACE score). Subsequently, the second order session*condition model was analyzed, stratified by the binary (early childhood and adolescent) ACE score variables. Figure 2 and 3 show the expected effect in that task reactivity was stronger in the high ACE group compared to low ACE group. For both groups, task reactivity was attenuated during the second session. In all stratified models, the session*condition effect was significant (all p's <.001) for LTM. Results for RTM were similar (all p's <.008). 


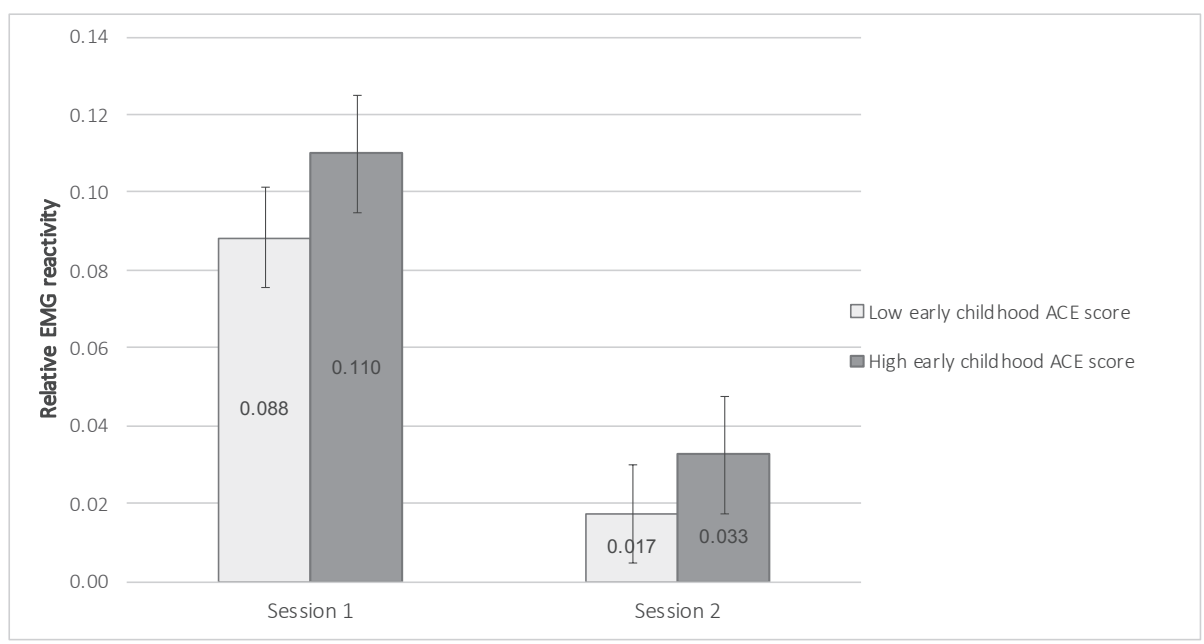

Figure 2. Task reactivity at session 1 and 2, comparing high and low early childhood ACE score categories

Note. Task reactivity was stronger in high ACE group compared to the low ACE group (session*condition effect significant<.001).

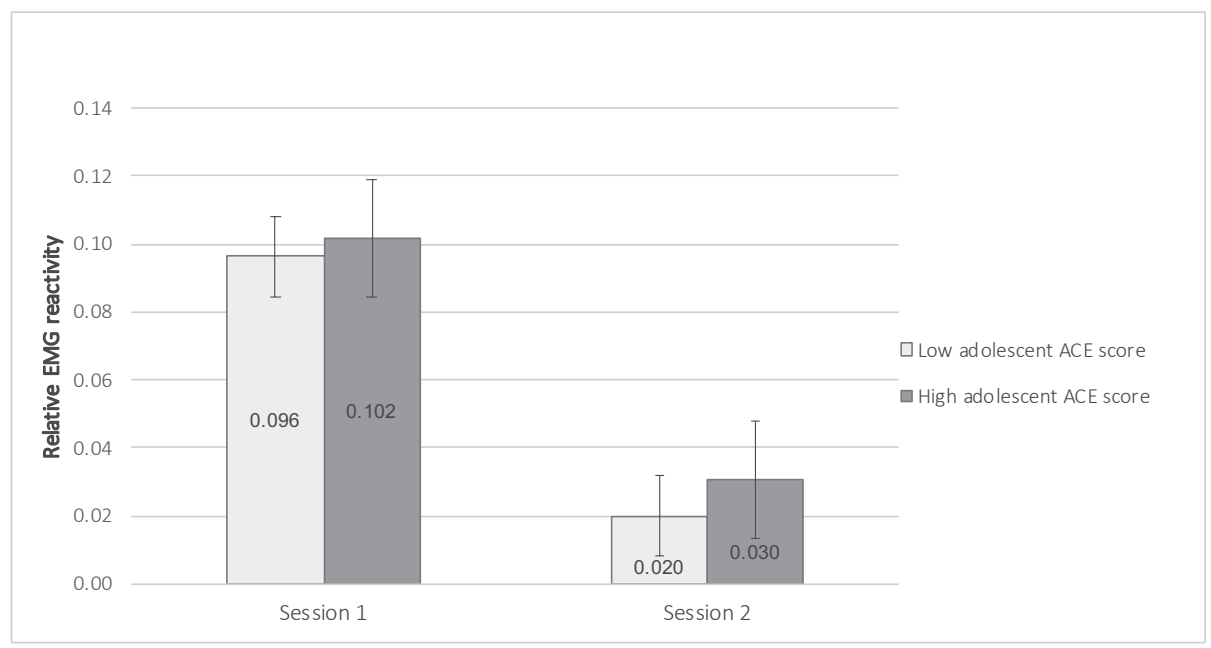

Figure 3. Task reactivity at session 1 and 2, comparing high and low adolescent ACE score categories Note. Task reactivity was stronger in high ACE group compared to the low ACE group (session*condition effect significant <.001). 


\section{DISCUSSION}

This study investigated the influence of ACE on EMG activity over time in a newly developed stress paradigm. The first objective of the study was to validate the experimental procedure. Compared to baseline, EMG reactivity was significantly higher during the memory task for both the left and the right trapezius muscle. As the baseline condition for recording EMG was sitting still and silent with eyes open and the experimental condition included the memory task, it could be argued that doing any task (i.e., the memory task) other than sitting still (i.e., the baseline condition) may increase EMG activity. However, combining the observed psychophysiological effect with the fact that all participants (subjectively) reported the task as stressful suggests that the memory task can be considered as a stress-inducing experiment. Nevertheless, follow-up should be conducted including a non-stressful control condition in order to definitively conclude that the observed EMG reactivity specifically indexes stress-reactivity.

Consistent with our a priori hypothesis, the condition*session interaction was furthermore found to be significant. Reactivity to the stressor (the memory task) was smaller in session 2 compared to session 1. The logical explanation for this finding is that participants were familiar with the task at session 2 and thus perceived less unpredictability and uncertainty than the first time. This is in accordance with early studies on human stress, demonstrating that controllability and predictability over an aversive event is less arousing compared to absence of these dimensions. ${ }^{31}$ The expected effect of reduced EMG activity with increasing age could not be observed, although the association ( $p=.099$ ) is suggestive of a trend in the hypothesized direction. Post-hoc, it was found that this may be related to the overrepresentation of higher educated people in the study population, possibly confounding the main effect of age.

Further, it is generally known that people differ in their EMG baseline level, as well as in their reactivity to stressors and in their stress reactivity over time. ${ }^{19}$ These notions were confirmed in this study given highly significant random intercept, condition and session effects across all analyses. The results indicate that participants differ regarding general (task irrelevant) EMG variability as well as task reactivity (condition effect) and between session variability. In multilevel analysis, main effects are corrected for these so called 'random' effects. The findings further support the notion that the memory task used in this experiment can be considered as a valid stress-inducing experimental paradigm.

The second objective was to investigate the generalizability of the finding reported by Luijcks and coworkers ${ }^{21}$ that ACE influences EMG stress reactivity in a stress experiment, in this case a memory task. This was demonstrated for the left trapezius muscle for 
early childhood ACE (0-11 years) as well as ACE during adolescence (12-17 years). The fact that we not only found an effect for early childhood ACE but also for adolescent $A C E$, is in line with other findings in the literature, confirming that ACE can have longlasting effects on the individual. ${ }^{1-3}$

In accordance with previous research demonstrating a dose-response relationship between ACEs and the risk of subsequent health problems, it was found that participants with higher ACE scores showed increased EMG reactivity relative to those with lower ACE scores.9.11 The impact of the stressor thus was greater for people with higher ACE scores. The negative condition*session effect indicates that individuals experienced less EMG reactivity at the second session. The third order interaction tested to what degree this finding was moderated by ACE score, which indeed was the case. Although EMG reactivity during the second session was lower compared to the first, for people with higher ACE scores (both early and adolescent) the impact of the condition*session effect was still greater compared to participants with lower ACE scores.

The third-order interaction may not appear in accordance with the finding by Luijcks and colleagues ${ }^{21}$ who did not report such an interaction. However, the reason they did not find a significant third-order interaction is probably related to the fact that in their stress experiment, participants received an unpredictable and uncontrollable electrical painful stimulus at both sessions. Hence, although participants knew what the stress experiment was about, they had no information about the timing nor the intensity of the electroshock that was administered at both sessions. Thus, the unpredictability of the experiment was similar 6 months later. In contrast, the procedure of the memory task was identical during the second session, thus reducing the unpredictability of the experiment.

The results of the present study may be generalized to real life situations as follows: confronted with an unpredictable or uncertain event, individuals who have experienced early adversity may react with stronger EMG reactivity compared to those who did not. However, in situations where there is no or little unpredictability, there may be no difference in stress reactivity between individuals who did or did not experience ACE.

The question may rise to what degree the ACE questionnaire indexes events that are 'adverse' or 'traumatic'. Although much research has been done in this area, definitions of 'trauma' and 'adversity' used in the literature are not consistent. ${ }^{32}$ A widely used definition of trauma, offered by the American Psychiatric Association, is: "Exposure to actual or threatened death, serious injury, or sexual violence, either by directly experiencing or witnessing such events or by learning of such events occurring to a close relative or friend". 33 
However, other approaches widen the concept of trauma, for instance the definition of Shapiro describing trauma as any event that has had a lasting negative effect upon self and psyche. ${ }^{34}$ This definition offers a more dimensional view of adversity, where trauma is positioned at the extreme end. ${ }^{35}$ McLaughlin proposed that childhood adversity should be defined as: "Experiences that are likely to require significant adaptation by an average child and that represent a deviation from the expectable environment". 32 Therefore, whereas traumatic events taking place in childhood may represent childhood adversity, not all types of childhood adversity may be considered traumatic. 'Non-traumatic' ACEs are for example poverty and the absence of a stable, supportive caregiver.

Taken together, the results of the current investigation can be taken to reflect the impact of ACE reflecting childhood adversity and not severe traumatic events at the extreme end of the distribution.

\section{Limitations of the study}

As noted earlier, follow-up research including a non-stressful control condition is required in order to definitively conclude that the observed EMG reactivity indexes stress-reactivity. Furthermore, a future improvement of the paradigm would be to also assess the subjective level of experienced stress during the experiment, for instance using a visual analogue scale (VAS). In the present study, this did not happen.

EMG was measured by two electrodes: one on the right trapezius muscle and one on the left. In future research, it may be productive to place more electrodes and/or attach electrodes to different body parts.

ACE was measured with a self-report questionnaire, which is known to be prone to several forms of bias such as recall bias and response bias. In the case of bias, effects may be underestimated or overestimated. However, whether in this case a structured interview would lead to more accurate results than a self-report questionnaire is not known, since it can entail the same types of bias. ${ }^{36}$

In future research, it may be productive to explore the timing of ACE in more detail; the two exposure periods used in this study (0-11 years and 12-17 years) are quite broad and arguably arbitrary. Assessing the experienced impact of ACE would furthermore be of value; the questionnaire used for the current experiment provided no information on this. 
It could be argued that the ACE questionnaire is very non-specific regarding the type of event and thus not a good tool to assess the impact of adverse events. We are aware of the fact that many factors contribute to the impact on an individual. In the end, what is considered 'adverse' or 'traumatic' is highly subjective and therefore nearly impossible to capture with a standardized measure. However, as already pointed out in the introduction, ACEs often co-occur and arguably are best viewed as a complex set of highly interrelated experiences rather than stand-alone events. ${ }^{13}$ In our study population, this also seems to be the case, given the fact that approximately $40 \%$ experienced two or more ACEs (table 1). Hence, the scale is thought to be adequate for use in the present study.

The assessment of recent life stressors was very limited and provided no information about the number of events or the experienced impact of the event. In future research, it is recommended to also collect this information.

All validation analyses yielded comparable results for both left and right trapezius muscle reactivity. However, the third-order interaction was significant only for the left trapezius muscle. The difference between the highly significant LTM non-significant RTM effects is of interest. Although we do not have a post-hoc explanation for this finding, there may be an underlying mechanism which requires further elucidation. Therefore, replication of the findings of the present study is required.

\section{Directions for future research and implications}

The memory task is straightforward and easy to perform, independent of personal factors like age, educational level and illness status. The presented paradigm may thus be used in future studies investigating the influence of ACE on stress and (mental) health.

This study was conducted in a general population sample. Examining specific clinical populations (e.g., people diagnosed with post-traumatic stress disorder, anxiety disorder or mood disorder) may provide more insight into the relationship between ACE, EMG stress reactivity and (mental) health problems. Furthermore, the memory task may also be used in a clinical setting, in order to assess psychophysiological stress reactivity in relation to treatment and prognosis.

Finally, in clinical practice, one of the issues that remains to be resolved is why certain individuals are resilient after exposure to adversity and others are not. The outcome of this study, as well as the study by Luijcks and colleagues ${ }^{21}$, suggests that differential resilience may be investigated using EMG reactivity as a stress marker. 


\section{Acknowledgments}

We want to thank Jacco Ronner for designing the memory task (department of psychology, Maastricht University) and Ron Mengelers for providing technical support (department of psychiatry and neuropsychology, Maastricht University). 


\section{REFERENCES}

1. Glaser JP, van Os J, Portegijs PJ, Myin-Germeys I. Childhood trauma and emotional reactivity to daily life stress in adult frequent attenders of general practitioners. J Psychosom Res Aug 2006;61(2):229-236.

2. Lardinois M, Lataster T, Mengelers R, Van Os J, Myin-Germeys I. Childhood trauma and increased stress sensitivity in psychosis. Acta Psychiatr Scand Jan 2011;123(1):28-35.

3. Bunce SC, Larsen RJ, Peterson C. Life after trauma: personality and daily life experiences of traumatized people. J Pers Jun 1995;63(2):165-188.

4. Carpenter LL, Shattuck TT, Tyrka AR, Geracioti TD, Price LH. Effect of childhood physical abuse on cortisol stress response. Psychopharmacology (Berl) Mar 2011;214(1):367-375.

5. Heim C, Newport DJ, Bonsall R, Miller AH, Nemeroff CB. Altered pituitary-adrenal axis responses to provocative challenge tests in adult survivors of childhood abuse. Am J Psychiatry Apr 2001;158(4):575-581.

6. Luijcks R, Vossen CJ, Hermens HJ, van Os J, Lousberg R. The Influence of Perceived Stress on Cortical Reactivity: A Proof-Of-Principle Study. PLoS One 2015;10(6):e0129220.

7. Teicher MH, Tomoda A, Andersen SL. Neurobiological consequences of early stress and childhood maltreatment: are results from human and animal studies comparable? Ann N Y Acad Sci Jul 2006;1071:313-323.

8. Wichers M, Schrijvers D, Geschwind N, et al. Mechanisms of gene-environment interactions in depression: evidence that genes potentiate multiple sources of adversity. Psychol Med Jul 2009;39(7):1077-1086

9. Valerie J. Edwards, George W. Holden, Vincent J. Felitti, Robert F. Anda. Relationship Between Multiple Forms of Childhood Maltreatment and Adult Mental Health in Community Respondents: Results From the Adverse Childhood Experiences Study. American Journal of Psychiatry 2003;160(8):1453-1460.

10. Anda RF, Felitti VJ, Bremner JD, Walker JD, Whitfield C, Perry BD, Dube SR, Giles WH. The enduring effects of abuse and related adverse experiences in childhood. A convergence of evidence from neurobiology and epidemiology. Eur Arch Psychiatry Clin Neurosci Apr 2006;256(3):174-186.

11. Hughes K, Lowey H, Quigg Z, Bellis MA. Relationships between adverse childhood experiences and adult mental well-being: results from an English national household survey. BMC Public Health Mar 3 2016;16:222.

12. Nakazawa DJ. Childhood disrupted : how your biography becomes your biology, and how you can heal. New York: Atria Books; 2015.

13. Dong M, Anda RF, Felitti VJ, Dube SR, Williamson DF, Thompson TJ, Loo CM, Giles WH. The interrelatedness of multiple forms of childhood abuse, neglect, and household dysfunction. Child Abuse Negl Jul 2004;28(7):771-784.

14. Labonte B, Suderman M, Maussion G, et al. Genome-wide epigenetic regulation by early-life trauma. Arch Gen Psychiatry Jul 2012;69(7):722-731.

15. Reich CG, Taylor ME, McCarthy MM. Differential effects of chronic unpredictable stress on hippocampal CB1 receptors in male and female rats. Behav Brain Res Nov 05 2009;203(2):264-269.

16. Levine S. What is stress? Stress, Neurobiology and NeuroEndocrinology 1991 1991:3-21.

17. Selye H. The nature of stress. Basal Facts 1985;7(1):3-11. 
18. Balodis IM, Wynne-Edwards KE, Olmstead MC. The other side of the curve: examining the relationship between pre-stressor physiological responses and stress reactivity. Psychoneuroendocrinology Oct 2010;35(9):1363-1373.

19. Lundberg U, Kadefors R, Melin B, Palmerud G, Hassmen P, Engstrom M, Dohns IE. Psychophysiological stress and EMG activity of the trapezius muscle. Int J Behav Med 1994;1(4):354-370.

20. Wijsman J, Grundlehner B, Penders J, Hermens H. Trapezius Muscle EMG as Predictor of Mental Stress. Acm T Embed Comput S Jun 2013;12(4).

21. Luijcks R, Vossen CJ, Roggeveen S, van Os J, Hermens HJ, Lousberg R. Impact of early life adversity on EMG stress reactivity of the trapezius muscle. Medicine (Baltimore) Sep 2016;95(39):e4745.

22. Willmann $\mathrm{M}, \mathrm{Bolmont} \mathrm{B}$. The trapezius muscle uniquely lacks adaptive process in response to a repeated moderate cognitive stressor. Neurosci Lett Jan 6 2012;506(1):166-169.

23. Cardozo AC, Gonçalves M, Hallal CZ, Marques NR. Age-Related Neuromuscular Adjustments Assessed by EMG. In: Turker H, ed. Electrodiagnosis in New Frontiers of Clinical Research. Rijeka: InTech; 2013:Ch. 06.

24. van Os J, Rutten BP, Poulton R. Gene-environment interactions in schizophrenia: review of epidemiological findings and future directions. Schizophr Bull Nov 2008;34(6):1066-1082.

25. McCrae RR. The five-factor model and its assessment in clinical settings. J Pers Assess Dec 1991;57(3):399-314.

26. Costa PT, Jr., McCrae RR. Stability and change in personality assessment: the revised NEO Personality Inventory in the year 2000. J Pers Assess Feb 1997;68(1):86-94.

27. Spielberger CD, Gorsuch, R.L., Lushene, R., Vagg, P.R. and Jacobs, G.A. Manual for the State-Trait Anxiety Inventory. Palo Alto: Consulting Psychologists Press; 1983.

28. Cohen S, Kamarck T, Mermelstein R. A global measure of perceived stress. J Health Soc Behav Dec 1983;24(4):385-396.

29. Noone P. The Holmes-Rahe Stress Inventory. Occup Med-Oxford Oct 2017;67(7):581-582.

30. Luijcks R, Hermens HJ, Bodar L, Vossen CJ, Van Os J, Lousberg R. Experimentally induced stress validated by EMG activity. PLoS One 2014;9(4):e95215.

31. Miller SM. Controllability and human stress: method, evidence and theory. Behav Res Ther 1979:17(4):287-304.

32. McLaughlin KA. Future Directions in Childhood Adversity and Youth Psychopathology. J Clin Child Adolesc May-Jun 2016;45(3):361-382.

33. Association AP. Diagnostic and Statistical Manual of Mental Disorders, 5th Edition (DSM-5) Washington, DC: American Psychiatric Press; 2013.

34. Shapiro F. The role of eye movement desensitization and reprocessing (EMDR) therapy in medicine: addressing the psychological and physical symptoms stemming from adverse life experiences. Perm J Winter 2014;18(1):71-77.

35. Seery MD, Holman EA, Silver RC. Whatever does not kill us: cumulative lifetime adversity, vulnerability, and resilience. J Pers Soc Psychol Dec 2010;99(6):1025-1041.

36. Hardt J, Rutter M. Validity of adult retrospective reports of adverse childhood experiences: review of the evidence. J Child Psychol Psychiatry Feb 2004;45(2):260-273. 




\section{CHAPTER 5}

\section{THE INFLUENCE OF CHILDHOOD ADVERSITY ON STRESS SENSITIVITY AND HABITUATION TO PAIN}

Marsman A, Luijcks R, Vossen C, van Os J, Lousberg R. The influence of childhood adversity on stress sensitivity and habituation to pain. 2021. 


\section{ABSTRACT}

Extensive evidence exists that childhood adversity can cause long-lasting detrimental effects. Increased stress sensitivity, representing an acquired vulnerability that can be traced back to early stress exposure, may form one of the mechanisms that (partially) explains how childhood adversity can cause health problems in adulthood. We explored this mechanism by investigating the relationship between adverse childhood experiences (ACE) and stress sensitivity in a stress-experiment that exposed a sample of 120 healthy participants to a series of 25 painful electrical stimuli, while measuring EMG activity. Additionally, we investigated the influence of ACE on habituation to pain. In order to assess the role of predictability, the study protocol included an identical session six months later.

Stress sensitivity was operationalized by measuring EMG stress-reactivity of the trapezius muscle and analyzed using multilevel regression analysis. We furthermore analyzed pain reports of each stimulus, measured with a numeric rating scale (NRS). Compared to baseline, EMG reactivity was higher during the habituation task, supporting the validity of the stress-experiment. Additionally, both a within-session and between-session habituation effect was observed: EMG decreased in the interstimulus-intervals as well as over the course of the 25 stimuli. Accordingly, NRS also decreased for each consecutive stimulus. As expected, EMG reactivity was attenuated during the second session. Analyses indicated a moderating effect of childhood adversity not only on EMG stress-reactivity, but also on the habituation to pain: higher ACE scores were associated with increased EMG reactivity and diminished habituation.

In conclusion, this study provided support for the hypothesis that childhood adversity may impact later stress sensitivity, resulting in greater EMG reactivity and diminished habituation when exposed to a physical stressor. 


\section{INTRODUCTION}

Much research has examined, and continues to examine, the long-term consequences of experiencing adversity early in life. The Adverse Childhood Experiences Study (ACE Study), conducted in the United States in the early 1990s, 'showed associations between many types of childhood adversity and numerous physical and mental health problems in adulthood. Although ACE were defined by a set of 10 different experiences describing forms of neglect, abuse and family dysfunction, it has been acknowledged that these ACE are not the only forms of childhood adversity impacting later health. Additional adverse experiences are natural disasters, bullying, community violence, living in poverty and social inequality. ${ }^{2.3}$ The experience and impact of childhood adversity can be highly variable not only in terms of the nature of the adverse event, but also its timing, frequency, predictability and severity. ${ }^{4}$ However, irrespective of the variability, ACE per definition share the commonality of being stressful, overwhelming and perceived as negative by the individual so exposed.2.5

Childhood adversity is not uncommon. In the ACE Study that assessed more than 17,000 adults, $64 \%$ of the respondents reported at least one ACE and 23\% reported three or more ACE. A more recent study in the US yielded similar results ${ }^{6}$, and although there seems to be slight variation in the prevalence of childhood adversity between different nations, comparable findings are widely observed.7-10 ACE tend to co-occur. Thus, when assessing the impact of childhood adversity, one should always take into account the fact that ACE are often co-occurring with other types of adversity and rarely represent standalone events. ${ }^{4}$ ACE furthermore can affect brain, mind and body, impacting neurobiological and immunological processes, as well as autonomic, endocrine and metabolic systems."1-14 Accordingly, childhood adversity has been identified as a risk factor for developing a wide range of physical and mental health issues later in life, including - but not limited to - chronic pain, cardiovascular disease, cancer, diabetes, depression, psychosis, addiction and eating disorders. ${ }^{1521}$ Finally, there is extensive evidence for a strong dose-response relationship between number of ACE and increased risk for the aforementioned health problems.11.22 A study by Brown and colleagues, assessing more than 17,000 respondents, revealed that people with 6 or more ACE on average died nearly 20 years earlier than those without..23

\section{Increased stress sensitivity}

Several mechanisms have been proposed to explain how childhood adversity may cause health problems later in life. Non-mutually exclusive variation exists across theoretical frameworks, some relying primarily on biological mediation while others have a more psycho-social perspective. However, there is broad consensus that (1) ACE by definition are stressful events, and (2) stress can have a disruptive impact on child development..24,25 
From a biological point of view, there is evidence that strong, frequent and/or prolonged exposure to stress (i.e., toxic stress) can cause neurobiological alterations, contributing to heightened sensitivity to stress that persists throughout life.26,27 It thus may be hypothesized that a history of childhood adversity results in increased stress sensitivity, making an individual more vulnerable to the impact of daily life stressors, which eventually can contribute to a broad range of adverse health outcomes in adulthood.

Several studies exist that offer support for this hypothesis. For instance, findings from a 10-year prospective cohort study that investigated the synergism between early and recent adversity in people suffering from psychosis, suggest that early adversity may impact on later expression of psychosis either by increasing exposure to later adversity and/or by rendering individuals more sensitive to later adversity. ${ }^{28}$ Support is also offered by other studies that operationalized stress sensitivity as emotional reactivity to daily life stressors, $18,29-31$ demonstrating a significant association between ACE and increased sensitivity to stress.

In other words, stronger emotional reactions were observed to small stressors in daily life in people who had been exposed to ACE. Additionally, two other studies investigated the impact of childhood adversity at the physiological level, investigating electromyography (EMG) stress reactivity of the trapezius muscle during a stressexperiment. ${ }^{32,33}$ Both studies found that participants with higher ACE scores showed increased EMG reactivity relative to those with lower ACE scores.

Taken together, there is substantial evidence supporting the hypothesis that exposure to stress early in life results in increased sensitivity to stress, which, consequently, may heighten the vulnerability to disruptions in emotional and/or physiological regulation when exposed to subsequent, daily life stressors. ${ }^{34}$

In the present study, we explored the relationship between childhood adversity, heightened stress sensitivity and manifestations thereof by investigating stress-reactivity to painful stimuli in an experimental setting. Since there is an extensive body of literature showing that childhood adversity is a risk factor for developing various types of chronic pain conditions, ${ }^{35,36}$ for which mechanistic evidence is, however, lacking, we moreover investigated the influence of childhood adversity on habituation to pain. Similar to the studies conducted by Luijcks ${ }^{33}$ and Marsman, ${ }^{32}$ stress sensitivity was operationalized by measuring EMG stress-reactivity of the trapezius muscle. As it is known that muscle activity increases during stressful situations and the observations that the trapezius muscle is particularly susceptible to stress, ${ }^{37,38}$ it may represent a useful parameter for examining the influence of childhood adversity on stress-reactivity to pain. 
While earlier experimental studies on EMG stress-reactivity used a single painful stimulus or a cognitive stressor, the current study used a series of painful electrical stimuli, thereby allowing us to investigate how childhood adversity may impact habituation. In addition to EMG, we furthermore measured individual pain reports (using a numeric rating scale ranging from 0 - 100) of each administered stimulus, enabling the exploration of habituation on a more subjective level as well.

As the protocol used in this study was, to the best of our knowledge, not used before in combination with EMG, a first objective of the study was to validate the experiment as stress-inducing and, furthermore, measuring a form of habituation. Habituation, in this respect, refers to the phenomenon of an observed decrease in the response to a repeated stimulus. ${ }^{39}$ Accordingly, it was expected that both the subjective pain report of each stimulus as well as the overall EMG reactivity would decrease over the course of the experiment. As research suggests that it is the degree of unpredictability of a stressor that mediates its consequences, ${ }^{40}$ we also investigated the role of predictability of the experimental stressor by repeating the experiment six months later. Given reduction of unpredictability at the second session, we expected to find smaller moderating effects on EMG at the six-months follow-up. With respect to pain report, however, no session effect was expected, assuming that NRS scores of the administered stimuli were not generally influenced by predictability of the experiment, but primarily by the experienced intensity of the stimulus. Importantly, the intensity of the stimulus was calculated for each participant separately at the start of both sessions, such that it was experienced as painful yet tolerable.

Next, the following and primary objectives of the study were to (1) investigate the relationship between childhood adversity and EMG stress-reactivity (i.e., stress sensitivity), and (2) investigate the relationship between childhood adversity and habituation to pain, measured as EMG stress-reactivity as well as pain reports. In line with the findings by Luijcks ${ }^{33}$ and Marsman, ${ }^{32}$ we hypothesized that exposure to childhood adversity would be associated with increased EMG activity during the experiment. In our study, a questionnaire measuring ACE in two specific age periods (i.e., early childhood and adolescence), was used to operationalize childhood adversity. As the literature suggests that exposure to stress is most impactful during early childhood, ${ }^{5}$ we expected to find stronger associations with early childhood ACE as compared to adolescent ACE. It was furthermore hypothesized that any moderating effect of ACE would be smaller at the second session six months later.

With regard to the habituation process, we hypothesized that exposure to ACE (compared to no ACE) would be associated with diminished habituation to pain, expressed as a slower decrease of EMG-reactivity and a slower decrease of NRS scores over the course of the experiment. 
Based on previous research regarding factors that can impact stress-reactivity and habituation to pain, a set of potential confounders was included in all models, namely: age, gender, NEO-neuroticism scale, anxiety (STAl- state version), perceived stress (PSS), recent life events, current pain-complaints, pain threshold, sensation threshold and pain report. These variables are explained in more detail in the method section. 


\section{METHODS}

\section{Participants}

The experiment, referred to as the 'habituation task', was approved by the medical ethics committee of the Academic Hospital Maastricht and Maastricht University (NL40284.068.12/METC 12-3-015). The experiment was part of a larger study that evaluated psychophysiological reactivity as a predictor of change in pain complaints. All participants provided written informed consent before the start of the experiment. One-hundred and twenty participants (78 women and 42 men) participated in the study. Age ranged from 18 to 66 years (mean 40.5, SD 17.1). The sampling frame was a general population sample derived from the population living in the city of Maastricht, the Netherlands. Exclusion criteria were structural use of antipsychotics, antiepileptics or anxiolytics during the past year or structural use of alcohol in excess of $7 \mathrm{u} /$ day. Participants were asked to refrain from alcoholic beverages the evening before the experiment, and to refrain from caffeine-containing consumptions three hours prior to the experiment. Participants received $€ 50$ for participating in the study.

\section{Procedure}

Upon arrival in the laboratory, participants were first asked to complete a set of questionnaires, after which EMG-, and ECG-electrodes as well as the shock electrode were attached. EMG electrodes were attached on the left and right trapezius muscles (LTM and RTM, respectively). The baseline measurement was 5 minutes, in which participants were instructed to sit as still as possible, with their eyes open. Next, the individual sensation and pain threshold was determined. Participants were informed that they would receive a series of stimuli that could slightly vary in intensity and were instructed to determine the differences between each stimulus. Both the intensity and number of stimuli were unknown to the participant. They were asked to verbally rate the intensity of each stimulus on a numeric rating scale (NRS) from $\mathrm{O}$ (no sensation) to 100 (the most severe pain imaginable). For standardization purposes, participants were asked to score the first stimulus as 60. Participants were instructed to keep both hands on the table, palms down, and to not close their eyes during the entire experiment. The study protocol included an identical follow-up measurement 6 months after the first session.

\section{Electroshocker and stimuli}

An electroshocker (Shocko-100-AA-20, developed by Maastricht Instruments BV and approved for use in experimental studies) was used to apply the electrical stimuli (see also Vossen et al. ${ }^{41}$ and Luijcks et al..$^{33}$ ). Electrical pulse stimuli of 10 milliseconds duration were delivered intracutaneously to the left middle finger, according to a method 
described by Bromm and Meier. ${ }^{42}$ Sensation and pain thresholds were determined for all subjects individually by starting at zero intensity and gradually increasing by steps of $0.05 \mathrm{~mA}$. The first consciously experienced intensity was defined as the sensation threshold. The pain threshold was defined as the first intensity considered as painful, but still acceptable. The maximum stimulus intensity never exceeded $5 \mathrm{~mA}$. To generate reliable estimates of the thresholds, this procedure was carried out three times in total.

\section{Habituation protocol}

Based on a participant's difference between the sensation and pain thresholds, a stimulus that was 25\% above the pain threshold was calculated as follows:

Delivered habituation stimulus = pain threshold $0.25^{*}$ (pain threshold - sensation threshold $)$

The intensity of the administered stimulus was experienced as painful yet tolerable. The protocol consisted of 25 identical stimuli of 10 ms duration. The interstimulus intervals (ISIS) varied between 9 and 11 seconds. The procedure was controlled by the software program 'Presentation 0.71' (Neurobehavioral Systems, Berkeley, California, USA).

\section{Psychophysiological recordings}

EMG activity of the left and right upper trapezius muscle was recorded in an electrically and sound-shielded cubicle (7.1 $\left.\mathrm{m}^{2}\right)$, using silver/silver chloride $(\mathrm{Ag} / \mathrm{AgCl})$ electrodes. Electrodes were centered on a point $2 \mathrm{~cm}$ lateral to the midpoint between the acromion process and spinous process of the seventh cervical vertebra. A reference electrode was placed over the spinous process of the seventh cervical vertebra. Cardiac activity was recorded with a standard 3-lead ECG. Conductive paste (Ten20) was used to fix all electrodes. Brainvision BrainAmp Research Amplifier was used for all recordings. ECG and EMG were sampled with $1000 \mathrm{~Hz}$.

\section{Psychological measurements}

Prior to the habituation task, participants were asked to complete a set of questionnaires (described below) in an adjacent room in the laboratory. Additional questions were asked concerning participants characteristics (e.g., age, sex) and current pain complaints.

Adverse childhood experiences (ACE)

ACE, representing childhood adversity, were assessed with a questionnaire developed within the context of the FP7 EU-GEl project (European Network of National Schizophrenia Networks Studying Gene-Environment Interactions) ${ }^{43}$. The questionnaire comprises 15 yes/no questions on adverse childhood events, such as abuse, neglect and divorce of parents. The questionnaire covers two exposure periods: the first includes 
exposure between 0 and 11 years (early childhood), the second period between 12 and 17 years (adolescence). Prior research has shown adequate internal consistency for both age periods. ${ }^{33}$ The maximum score in each age period was 15 , the maximum score for the entire questionnaire was 30. The sum of scored events for both age periods together ranged from 0 to 11 (mean 2.3, SD 2.5). For the exposure period of 0 to 11 years, the sum of scored events ranged from 0 to 7 (mean 1.2, SD 1.5), whereas for the exposure period of 12 to 17 years, the sum of scored events ranged from 0 to 6 (mean 1.1, SD 1.3)

\section{Neuroticism}

Assessed with the Revised Neuroticism-Extraversion-Openness Personality Inventory (NEO-PI-R). The NEO-PI is a 240-item self-rating questionnaire that measures five major personality dimensions. Items are scored on a five-point Likert scale, ranging from 1 (disagree strongly) to 5 (agree strongly). The questionnaire is widely used to operationalize the five-factor model of personality ${ }^{44}$ and has been validated extensively. ${ }^{45}$ From the NEO-PI-R, a total neuroticism score was calculated, representing a tendency or predisposition to experience negative affective states such as depression, anger, anxiety and impulsiveness. The total score ranged from 80 to 200 (mean 130, SD 22.0).

\section{State anxiety}

Assessed with the State-Trait Anxiety Inventory (STAI), a commonly used measure of trait and state anxiety. ${ }^{46}$ The questionnaire consists of 20 items assessing trait anxiety and 20 for state anxiety. The State Anxiety scale assesses the current anxiety state, using items such as 'I am tense', rated on a 4-point Likert scale ranging from 1 (almost never) to 4 (almost always). Higher scores indicate greater anxiety. The total score ranged from 20 to 76 (mean 36.6, SD 10.1).

\section{Perceived Stress}

Assessed by the Perceived Stress Scale (PSS), a 10-item questionnaire on feelings and thoughts during the last month. The PSS is the most widely used instrument with acceptable psychometric properties for measuring the perception of stress in daily life. ${ }^{47}$ Items are rated on a 5-point Likert scale ranging from 0 (never) to 4 (very often). A higher level of stress is indicated by higher scores on this scale. Total score ranged from 2 to 39 (mean 11.8, SD 6.1).

\section{Recent life events}

Assessed by asking participants whether or not they had experienced one or more events that impacted their daily lives in the previous year. To give participants an idea of what should be regarded a 'life event', a few examples from the Holmes-Rahe Stress 
Inventory ${ }^{48}$ were provided. Instead of using the entire Holmes-Rahe Stress Inventory, the assessment only consisted of a yes/no question in order to keep the experiment as short as possible. Overall, 86\% of the participants experienced at least one life event in the previous year.

\section{Offline data processing}

EMG data was filtered offline (low pass $0.5 \mathrm{~Hz}$, high pass $250 \mathrm{~Hz}, 50 \mathrm{~Hz}$ notch filter) and segmented into segments of 10 seconds. Raw data were visually inspected for artifacts using software (BrainVision Analyzer 2.0) and excluded from further analyses when detected. EMG activity was corrected for cardiac activity: variance due to ECG activity was removed from the uncorrected EMG variable, using regression analysis. Next, for each 10 second segment, the root mean square (RMS) value was calculated followed by a $10 \mathrm{log}$ logarithmic transformation to preserve a normal distribution. Consequently, the 5-minute baseline period was divided into 30 segments and the habituation task in 25 segments. This dataset was used for the first set of validation analyses. A second dataset, containing only habituation task data, was constructed in order to investigate the habituation effect. For this purpose, each stimulus, including a 9 second interstimulus interval, was divided in 500ms segments. Thus, this dataset consisted of 18 segments per stimulus, for a total of 25 stimuli. Last, a third dataset was constructed to specifically analyze effects on pain report, containing the 25 stimuli per session and the corresponding pain report of each stimulus.

\section{Statistical analysis}

The datasets had a hierarchical structure consisting of repeated time segments (level 1), nested within experimental sessions (levels 2), that are clustered within individuals (level 3). Given this structure, multilevel regression analyses were performed. In all models testing EMG, EMG activity of the LTM served as the dependent variable. While we also measured EMG activity of the RTM, we only hypothesized findings with respect to LTM, given the fact that electrical stimuli were administered to the left finger which we therefore a priori assumed would result in the LTM being primarily impacted. RTM findings were described post-hoc. To preserve normal distribution, the EMG variable was log-transformed. In order to test which covariance structure should be applied for both datasets, various covariance structures were tested. In agreement with earlier work with comparable experiments ${ }^{33,37}$, AR1 yielded the best fit.

We executed three sets of analyses; a series of validation analyses, a series of analyses to investigate the habituation effect, and finally a series of analyses to investigate the influence of childhood adversity on EMG reactivity and habituation to pain. Given the assumption that habituation differs between individuals, random effects, such as a 
random intercept and a random slope for stimulus number and session, were included in all models. All statistical analyses were performed using SPSS 26.0. P-values $\leq 0.05$ were considered to be statistically significant.

Validation analyses

First, we executed the validation analyses to test whether the habituation task could be considered a stress-experiment (i.e., stress-inducing) and, furthermore, whether the habituation task indeed measured a form of habituation. To this end, in a model with EMG as dependent variable, we tested a main task effect contrasting baseline (coded ' $\mathrm{O}$ ') with the habituation task (coded ' 1 ') and a task*session interaction effect (where session was coded ' $O$ ' at $T_{0}$ and ' 1 ' at $T_{1}$ ). Additionally, age (in years) and sex were included as predictor variables, as well as the NEO-neuroticism scale, STAI (state version), perceived stress (PSS), life events in the previous year, current pain-status, sensation and pain threshold. With respect to the habituation effect, we tested a main stimulus number effect, as well as a stimulus number*session interaction.

\section{Habituation analyses}

In order to examine the habituation effect, the dataset containing the variables 'stimulus number' (1-25) and 'segment number' (1-18) was used to model time effects. In addition to a linear time effect, an inverse effect (1/segment number) was also added. Habituation analyses were similar to the validation analyses described above. First, we tested main stimulus number and segment number associations, followed by analyzing time*session interactions (i.e., between-session habituation). Next, we analyzed the direct post-stimulus reactivity of EMG within the habituation task by testing a stimulus number*inverse segment number interaction (i.e., within-session habituation). In addition, we also investigated habituation on a more subjective level, by testing a main stimulus number and main session effect, as well as a stimulus number*session interaction, in a model with pain report (NRS) as dependent variable. For these analyses, the third dataset was used.

\section{ACE analyses}

To answer our primary research questions regarding the influence of childhood adversity on EMG reactivity and habituation to pain, in subsequent analyses ACE-score for the early childhood period and the adolescent period were included as predictor variables, in separate models. The following third-order interactions were of main interest with respect to EMG as dependent variable: ACE*task*session, ACE*stimulus number*session, ACE*segment number *session, ACE*stimulus number*segment number. 
With respect to the influence of ACE on pain reports, as we were more generally interested in investigating whether a history of ACE results in higher NRS scores compared to no ACE, we calculated a combined ACE-score (early ACE + adolescent ACE) that was dichotomized in $0=$ no ACE vs $1=$ ACE groups. Finally, we tested the ACE*stimulus number and ACE*stimulus number*session interaction. 


\section{RESULTS}

Due to technical issues with the electroshocker and/or electrodes, 6 subjects were excluded from the analyses, leaving a total of 115 analyzable participants (75 female, 40 male). At the second experimental session, there was attrition of 16 participants. General characteristics of the sample are displayed in table 1.

\section{Validation analyses: testing stress induction}

The central validation hypothesis was tested, expecting a significant EMG increase during the habituation task compared to baseline. A significant EMG increase was indeed demonstrated during the habituation task compared to baseline by a main task effect $(t=8.225, p<.001)$, indicating that the habituation task induces more stress compared to the baseline condition. Next, we investigated whether there was a task*session interaction, expecting to find lower EMG reactivity at session 2 compared to session 1. A significant interaction was found ( $t=-8.057, p<.001)$, as illustrated in figure 1. Post-hoc, it was investigated whether baseline level EMG differed between the two sessions (figure 1). However, this was not the case, as indicated by a non-significant association with session for both LTM $(p=.527)$ and RTM $(p=.120)$.

As described in the method section, EMG data were 10log-transformed. In order to calculate the EMG task effect in its original (untransformed) scale, a back-transformation was performed. In its original scale, EMG task reactivity was 1.45 times larger at $T_{0}$ than at $T_{1}$.

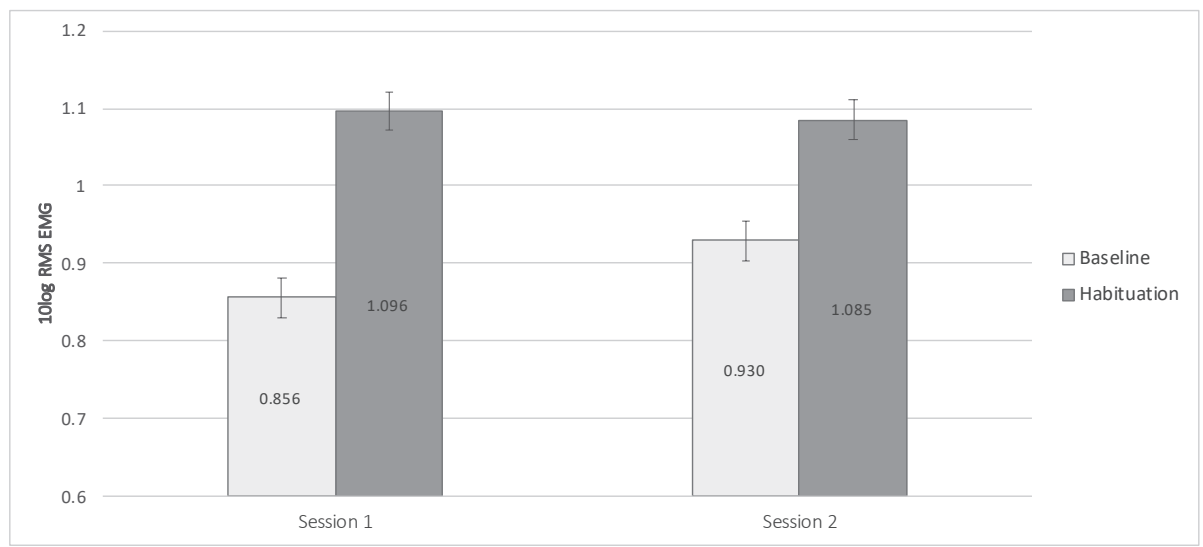

Figure 1. Predicted mean EMG (10log RMS) of the left trapezius muscle

Note. Mean EMG was higher during the habituation task at both sessions. Task-reactivity was greater at session 1 compared to session 2 (task*session interaction significant $p<.001$ ). 
Table 1. Descriptive statistics

\begin{tabular}{|c|c|c|}
\hline Variables & Session $1(N=115)$ & Session $2(N=99)$ \\
\hline Age, $\mu$ (s) min-max & $39.1(17.2) 18-66$ & $42.1(17.2) 18-66$ \\
\hline Sex: male/female (\%) & $35.0 / 65.0$ & 37.6 / 63.4 \\
\hline Life event(s) past year: yes / no (\%) & $86.4 / 13.6$ & $85.8 / 14.2$ \\
\hline Current pain-complaints: yes / no (\%) & $15.6 / 84.4$ & $29.4 / 70.6$ \\
\hline ACE: yes / no (\%) & $73.8 / 26.2$ & $70.6 / 29.4$ \\
\hline \multicolumn{3}{|l|}{ Early ACE } \\
\hline None & 39.9 & 40.2 \\
\hline 1 & 33.9 & 36.0 \\
\hline 2 & 8.7 & 6.9 \\
\hline$>2$ & 17.5 & 16.9 \\
\hline \multicolumn{3}{|l|}{ Adolescent ACE } \\
\hline None & 38.9 & 43.2 \\
\hline 1 & 33.9 & 33.1 \\
\hline 2 & 8.7 & 13.7 \\
\hline$>2$ & 17.5 & 10.0 \\
\hline STAI-state, $\mu(\mathrm{s})$ min-max & $36.6(10.1) 20-76$ & $35.1(9.2) 20-60$ \\
\hline PSS-score, $\mu$ (s) min-max & $11.8(6.1) 2-39$ & $12.0(5.7) 0-27$ \\
\hline NEO-neuroticism, $\mu$ (s) min-max & $130.5(22.0) 85-200$ & $129.4(22.4) 80-173$ \\
\hline Sensation threshold, $\mu$ (s) min-max & $7.1(4.0) 1-20$ & $7.21(4.3) 1-19$ \\
\hline Pain threshold, $\mu$ (s) min-max & $23.4(12.5) 3-52$ & $23.70(13.3) 4-65$ \\
\hline Current pain-complaints, $\mu$ (s) min-max & $.55(1.56) 0-8$ & $1.10(2.18) 0-8$ \\
\hline Pain report (NRS), $\mu$ (s) min-max & 57.5 (13.5) 5-100 & $55.21(14.4) 5-100$ \\
\hline
\end{tabular}

Note. ACE, Adverse Childhood Experience; STAI, State-Trait Anxiety Inventory, state-subscale; PSS, Perceived Stress Scale; NEO, Neuroticism-Extraversion-Openness Personality Inventory, neuroticism-subscale; NRS, Numeric rating scale.

Finally, all models showed a significant random intercept, random task and random session effect (all $p<.001$ ), indicating that participants differed regarding general (task irrelevant) EMG variability as well as task-reactivity (task effect) and additionally displayed between-session variability.

\section{Validation analyses: testing a habituation effect}

To investigate a habituation effect (i.e., decreasing EMG over the course of the experiment), we tested a main stimulus number effect. As hypothesized a priori, in the main model 'stimulus number' was significantly and negatively associated with EMG $(t=-2.215, p=.029)$, indicating a significant linear decrease of EMG over the course of 25 stimuli. We furthermore investigated the role of predictability by analyzing a 
stimulus number * session interaction. Given that participants knew what to expect the second session and were thus assumed to show less EMG activity, we expected the linear habituation to be attenuated in the second session compared to the first. This effect was indeed demonstrated ( $t=5.211$, $p<.001$, figure 2 ). Similar to the analyses above, all models showed significant random effects (all $p<.001$ ).

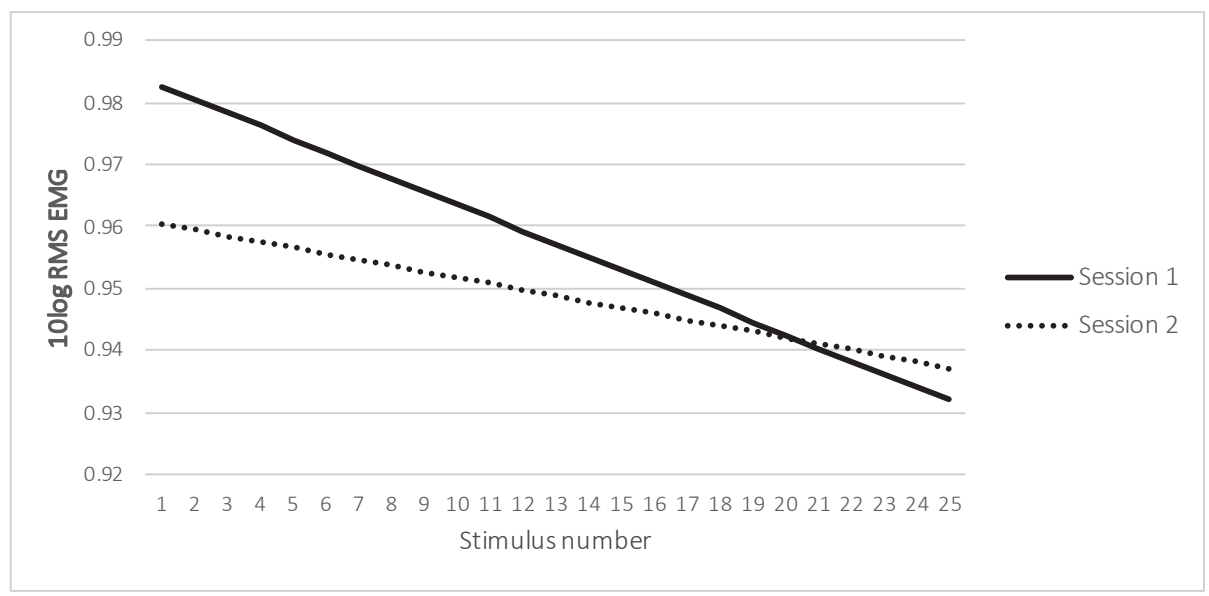

Figure 2. Modeled linear EMG activity, reflecting habituation over the course of 25 stimuli

Taken together, results of the validation analyses indicated that the habituation task represents a valid stress-experiment that furthermore measures a form of both withinsession and between-session habituation.

\section{Habituation analyses: EMG reactivity during the experiment}

In addition to a linear habituation effect (i.e., main stimulus number effect), we also tested a main inverse segment number effect, expecting a rapid initial decline of EMG values immediately after experiencing a stimulus, followed by a plateau phase. This effect was observed ( $t=6.663, p<.001)$ and is depicted in figure 3. Both the linear and inverse effects are indicative for habituation to the stimuli.

Additionally, we investigated whether there was an inverse segment number*session interaction, similarly expecting lower EMG reactivity in the inter-stimulus-intervals (ISIs) at session 2 compared to session 1. A significant interaction in this direction was found $(t=-13.416, p<.001)$, illustrating a between-session habituation effect. 


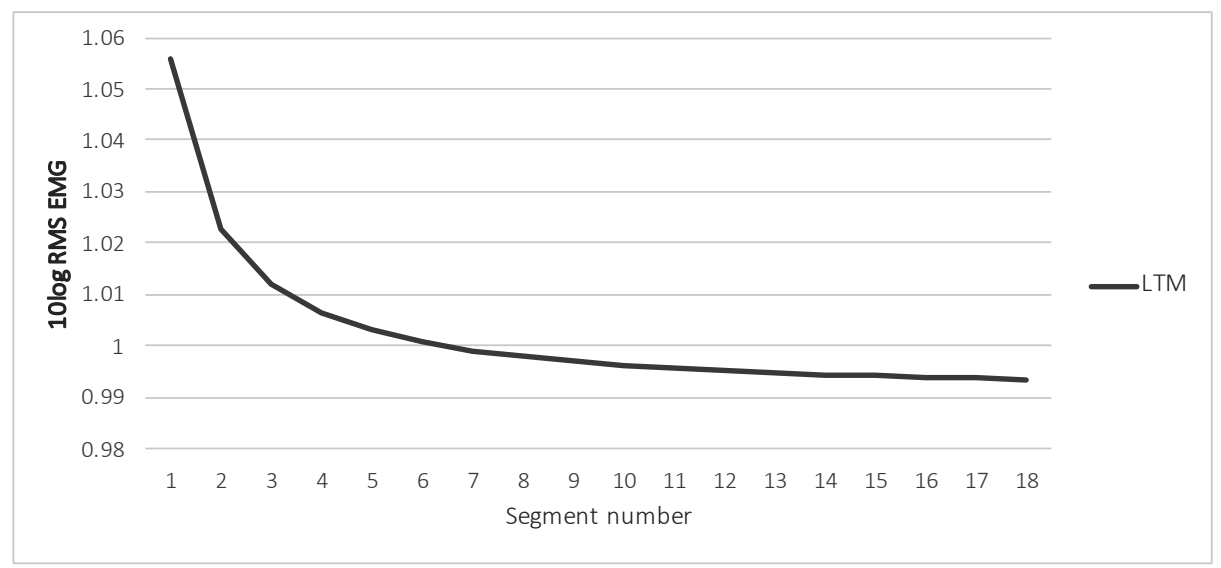

Figure 3. Modeled inverse EMG activity in left trapezius muscle (LTM) over the course of a single stimuli

\section{Post-stimulus EMG reactivity: segment number * stimulus number interaction}

We analyzed segment effects, i.e., the direct post-stimulus reactivity of EMG within the habituation task. This post-stimulus reactivity was earlier analyzed in combination with a 'long term' variable (session), observing a significant between-session habituation effect, described above. Analysis with a more short-term variable (stimulus number) yielded comparable results; a significant inverse segment number*stimulus number interaction was found $(t=-6.828, p<.001)$. Hence, a within-session habituation effect was also observed.

In accordance with the validation analyses, all models showed a significant random intercept, random session, random stimulus number and random inverse segment number effect (all $p<.001$ ), indicating that participants differed regarding general EMG variability as well as between stimulus, segment and session variability.

\section{Habituation on the level of pain report}

To investigate a habituation effect on a more subjective level, we tested a main stimulus number and main session effect in a model with pain report (NRS) as dependent variable. As expected, a significant stimulus number was observed ( $t=-3.155, p=.002$ ) demonstrating decreasing NRS over the course of the experiment. No main session effect ( $t=-1.195, p=.235$ ) as well as no session*stimulus number interaction ( $t=-.516$, $p=.606$ ) was found, indicating that the course of NRS did not differ between sessions. 


\section{Investigating the influence of childhood adversity}

The interaction between ACE and EMG task-reactivity

It was investigated whether the EMG task reactivity (task*session effect) was moderated by ACE score. Hence, for each ACE score (early childhood and adolescence) we ran a third-order interaction model with a task*session*ACE-score interaction variable. All models were adjusted for the set of confounders described in the method section, as well as random effects. The third-order interaction was significant for both the early childhood ACE-score $(\beta=-.100, t=-12.548, p<.001)$ and the adolescent ACE-score $(\beta=-.082, t=-9.094, p<.001)$.

In order to further explore the meaning of the third-order interaction, each ACE score was dichotomized at the median split ( 0 = relatively low ACE score, 1 = relatively high ACE score). Subsequently, the second order task*session model was analyzed, stratified by the binary (early and adolescent childhood) ACE score variables. For the early childhood group, the session*task effect was significant for both low and high ACE scores (all p-values <.003), the high ACE score showing a stronger effect. For the adolescent ACE group, a significant interaction was observed only for the high ACE score ( $p$ <.001) but not the low ( $p=.690$ ). Results are displayed in Table 2 and depicted in figure 4. It shows the expected direction of the association: task reactivity was stronger in the high ACE group compared to the low ACE group. For both groups, task reactivity was attenuated during the second session. The association was slightly stronger for early ACE.

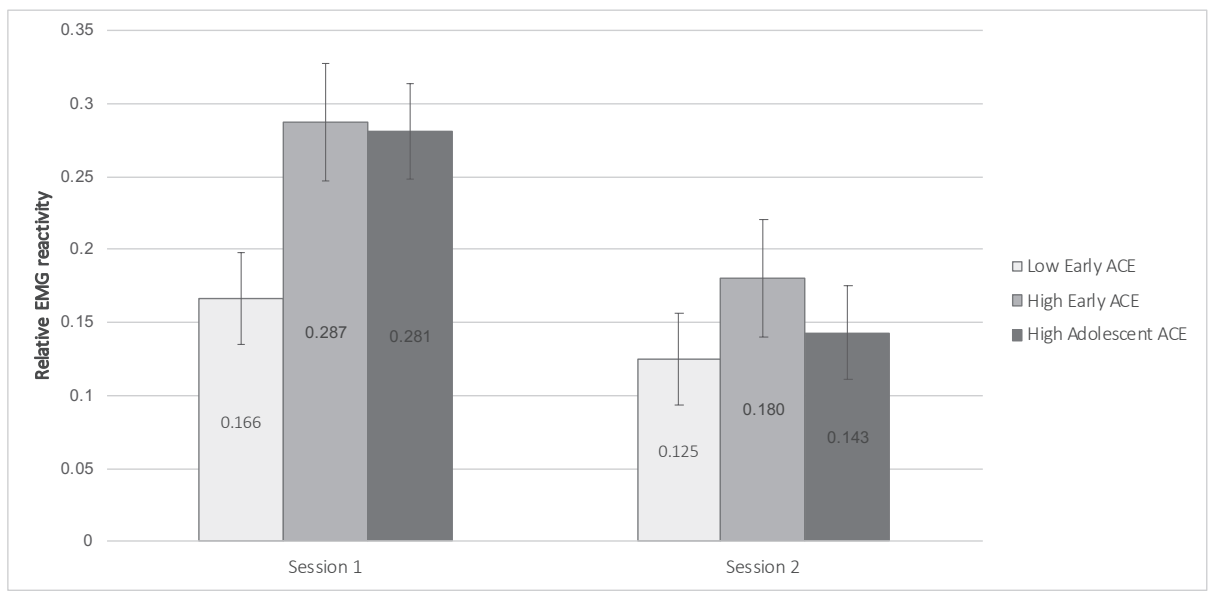

Figure 4. Task-reactivity at session 1 and 2, comparing high and low ACE score categories 
Table 2. Associations with EMG for left trapezius muscle

\begin{tabular}{llllll}
\hline Interaction & $\boldsymbol{\beta}$ & $\mathbf{S E}$ & $\mathbf{d f}$ & $\mathbf{t}$ & $\mathbf{p}$-value \\
\hline Session * task - Iow eACE & -.041 & .014 & 3925.940 & -3.024 & .003 \\
Session * task - high eACE & -.107 & .014 & 2625.115 & -7.449 & $<.001$ \\
Session * task - low aACE & -.005 & .013 & 4075.543 & -.393 & .690 \\
Session * task - high aACE & -.138 & .015 & 2841.114 & -9.106 & $<.001$ \\
\hline
\end{tabular}

Note. Session coded as $O=T_{0^{\prime}} 1=T_{1}$; task coded as $O=$ baseline, $1=$ habituation task; eACE, early ACE; aACE, adolescent ACE.

The interaction between ACE and habituation to pain

We examined to what extent ACE influences habituation to pain, using EMG activity as the dependent variable. First, it was investigated whether the main stimulus and inverse segment effects were moderated by ACE-score. No significant second order interaction was found between ACE and stimulus number and ACE and inverse segment number (all $p>.172$ for early ACE and $p>.153$ for adolescent ACE).

Next, we investigated whether the significant inverse segment*session, stimulus*session and inverse segment*stimulus interactions were moderated by ACE-score. For each ACEscore (early childhood and adolescence) we ran the following third-order interaction models: inverse segment*session*ACE-score, session*stimulus number*ACE-score and stimulus number*inverse segment*ACE- score. All models were adjusted for the set of possible confounders as described in the method section, allowing for random slopes. Results of significant associations with EMG are displayed in table 3. Stimulus number*inverse segment number*ACE interaction was not significant for both early ACE ( $t=-1.297, p=.195)$ and adolescent ACE ( $t=-.747, p=.455)$. The direction of the beta-coefficient is in accordance with our a priori hypothesis that childhood adversity negatively impacts habituation. This was observed for the short term (inverse segment effect) as well as for the 'longer' term (linear stimulus number effect).

Table 3. Significant associations with EMG of the left trapezius muscle

\begin{tabular}{llllll}
\hline Interaction & $\boldsymbol{\beta}$ & SE & df & t & p-value \\
\hline Session * Stimulus number * eACE & .001 & .000 & 9257.244 & 8.611 & $<.001$ \\
Session * Stimulus number * aACE & .001 & .000 & 9258.890 & 5.589 & $<.001$ \\
Inv segment number * Session * eACE & -.015 & .003 & 51833.557 & -5.702 & $<.001$ \\
Inv segment number * Session * aACE & -.024 & .003 & 48061.215 & -8.127 & $<.001$ \\
\hline
\end{tabular}

Note. Session coded as $O=T_{0}, 1=T_{1}$, eACE, early ACE; aACE, adolescent ACE; Inv, inverse. 
To further explore the meaning of the third-order interaction, each ACE score was dichotomized at the median split ( $O$ = relatively low ACE score, $1=$ relatively high ACE score). Subsequently, all significant second order interactions as described above were analyzed, stratified by the binary early and adolescent ACE score variables.

Session*Stimulus number: reflecting long-term habituation

Regarding early ACE, the session*stimulus number interaction was significant for the high ACE-group ( $t=6.115, p<.001$ ), but not for the low ACE-group ( $t=.012, p=.990$ ). For adolescent ACE, a significant interaction was observed for both the low $(t=-3.405$, $p<.001)$ and high ( $t=9.310, p<.001)$ ACE-group.

In order to accurately interpret these findings, it should be noted that, given the fact that EMG reactivity for the high ACE group is greater than for the low ACE group, there is more 'opportunity' for the high ACE group to show greater habituation (i.e., decreasing EMG reactivity). Furthermore, since in session 1 there is more unpredictability associated with the experiment, we expected to find reduced EMG activity at session 2. In order to conceptualize a 'pure' habituation effect, we thus needed to take into account the initial reactivity effect, as well as the session/predictability effect. To account for the reactivity effect, we calculated the difference between the first stimulus and the 24 consecutive stimuli. For the session effect, we calculated the difference between session 1 and 2 for each stimulus. This resulted in figure 5, visualizing the habituation effect over the sessions. As illustrated, high ACE scores resulted in decreased habituation, while low ACE scores resulted in increased habituation.

\section{Session*Inverse segment number: reflecting short term habituation}

Regarding early ACE, the inverse segment*session interaction was significant for both the low ACE-group $(t=-2.556, p=.011$ ) and the high ACE-group $(t=-15.315$, $p<.001)$. Similar results were observed for adolescent ACE, demonstrating a significant interaction for both the low ( $t=-4.156, p<.001)$ and high ( $t=-13.436, p<.001)$ ACE-group. In both cases, results demonstrated a stronger effect for the high ACE-group. In line with results regarding long term habituation described above, these results suggest that people with high ACE scores show a less rapid decline of EMG activity, hence, a diminished short-term habituation.

\section{The influence of ACE on pain report}

The influence of ACE on pain report, as measured on NRS, was examined. A basic model was used, in which NRS was the dependent variable and modeled as a function of stimulus number, session and the set of possible confounding variables as described in the method section. We tested a stimulus number*ACE interaction, finding that NRS 
scores were modified by ACE, such that participants with a history of ACE reported higher NRS scores relative to those without ACE ( $t=2.276, p=.025)$. For illustrative purposes, an overall course of NRSs across the 25 stimuli was constructed for the two ACE groups (ACE vs no-ACE) as depicted in figure 6. Subsequently, we tested a stimulus number*session*ACE interaction to see whether this result differed between the two sessions. As expected, this was not the case, indicated by a non-significant interaction $(t=-.976, p=.334)$.

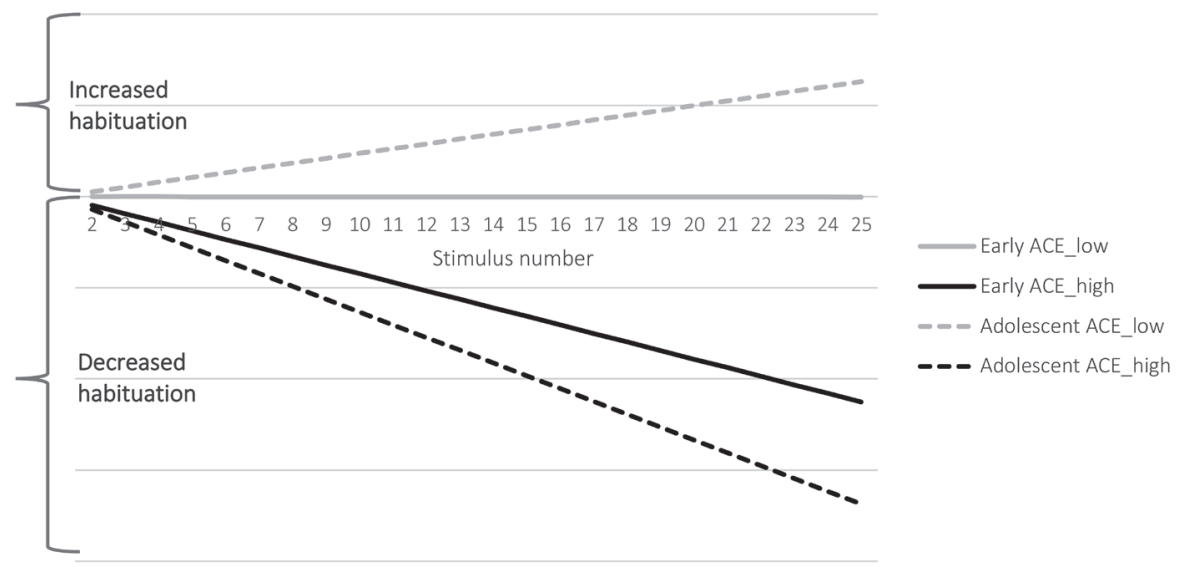

Figure 5. Conceptualized long-term habituation effects for low and high early ACE groups

Note. Both early and adolescent high ACE-score results in decreased habituation. Low early ACE interaction was not significant $(p=.990)$, all other interactions were significant $(p<.001)$

\section{Post-hoc RTM analyses}

Post-hoc, we ran all models presented above with RTM as the dependent variable. While most analyses yielded comparable results, for some analyses including interactions with ACE a discrepancy between results for LTM and RTM was observed (i.e., finding non-significant associations for RTM). We investigated whether this could be explained by outliers, however, this was not the case. This non-hypothesized contrast will be elaborated on further in the discussion. 


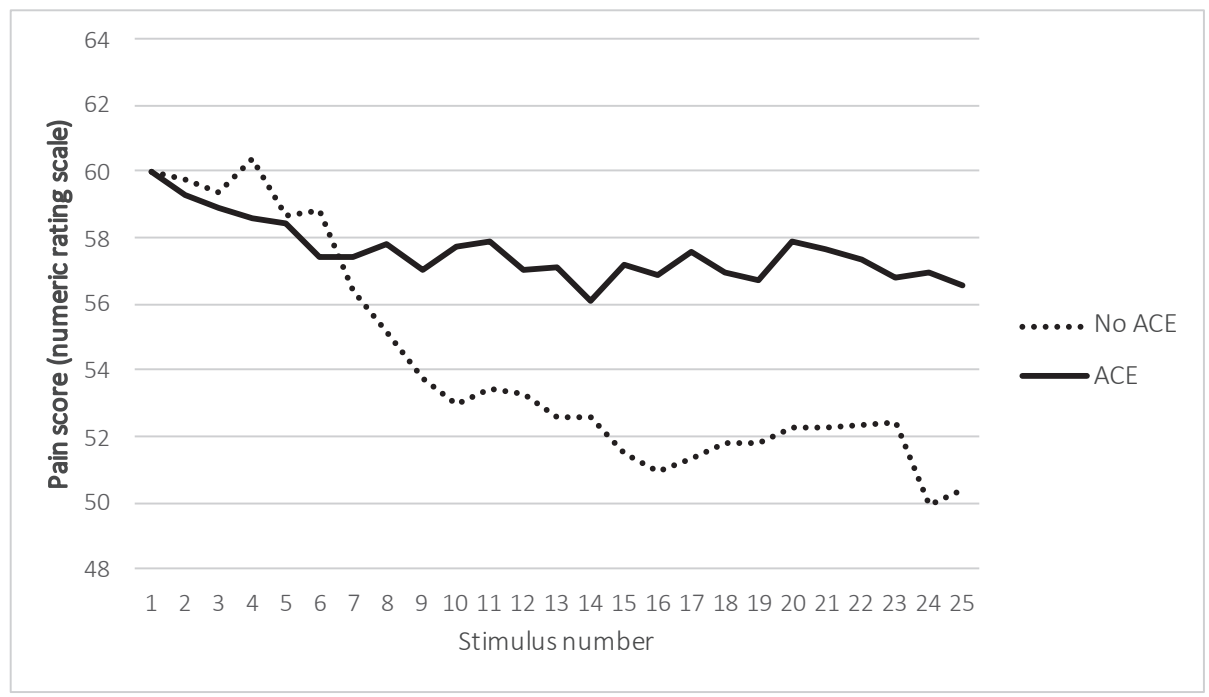

Figure 6. Numerical rating scale (NRS) course of 25 stimuli for participants with and without ACE 


\section{DISCUSSION}

We investigated the relationship between childhood adversity, heightened stress sensitivity and manifestations thereof by investigating EMG stress-reactivity to painful stimuli in an experimental setting. We furthermore investigated the impact of childhood adversity on habituation to pain, both on an objective (EMG) and subjective (pain report, NRS) level.

\section{Summary of findings}

The first objective of this study was to validate the stress-experiment. Results indeed showed that, compared to the baseline condition, EMG reactivity was significantly higher during the habituation task. This was the case in both sessions. As hypothesized, overall EMG reactivity was attenuated the second time, arguably because of decreased unpredictability of the experiment. Furthermore, the expected habituation-effect was also demonstrated: we found a main 'stimulus number' effect on EMG, indicating a linear decrease of EMG over the course of 25 stimuli, and a significant stimulus number*session interaction that indicated a diminished effect the second session.

With respect to habituation, next to a linear effect on EMG, we also found a main inverse segment number effect concerning the inter-stimulus-intervals, representing a rapid initial decline of EMG immediately after exposure to the stimulus, followed by a plateau phase. In accordance with the other findings, an inverse segment number*session effect was observed, representing a between-session habituation effect. We further demonstrated an inverse segment number*stimulus number interaction, indicating reduced EMG reactivity for increasing stimulus number, hence, within-session habituation. Last, in line with the EMG-findings, a habituation effect was also demonstrated on the subjective level of pain report, as indicated by a main stimulus number effect showing decreased NRS over the course of the experiment. As hypothesized a priori, this effect did not differ between sessions.

In addition to validating the habituation task as a stress-experiment that measures a form of habituation, the primary objectives of this study were to investigate the relationship between childhood adversity and EMG stress-reactivity, moreover, between childhood adversity and habituation to pain. Findings revealed that, generally, higher ACE-scores are associated with increased EMG reactivity to the experiment. As hypothesized, the moderating effect of ACE was attenuated at the second session, yet still significant. This effect was observed for both early childhood and adolescent ACE, the association being slightly stronger for the early childhood group. 
With respect to habituation on the EMG level, we found two significant third-order interactions (session*stimulus number*ACE and inverse segment number*session*ACE) for both early childhood and adolescent ACE. These results indicated that higher ACEscores are associated with diminished habituation, both in the short term (i.e., at the ISI-level) as well as the longer term (i.e., at the stimulus number level), and moderating both a within-session as well as a between-session habituation effect. In other words, results suggest that people with high ACE scores show a less rapid decline of EMG activity relative to those with a low ACE score. The short-term habituation was affected most by early childhood ACE, while adolescent ACE yielded a stronger association in the longer term.

With respect to habituation on the level of pain report, we found that NRS scores were also significantly moderated by childhood adversity, such that on average, people with a history of ACE reported higher NRS scores compared to people without ACE. Furthermore, over the course of the experiment these scores did not decrease as much as for the no-ACE group. As expected, the moderating effect of ACE on pain report did not differ between the two sessions.

Finally, all models showed significant random effects, indicating that participants differ regarding their EMG baseline level, as well as in their reactivity to stressors over time.

\section{Interpretation of findings}

The findings of this study are in line with previous studies demonstrating that higher ACE-scores are associated with increased EMG reactivity in experimental stresstasks. ${ }^{32,33}$ Moreover, this study demonstrated that habituation to a series of painful stimuli is negatively impacted by a history of ACE, both on the objective level of EMG, as well as on a more subjective level of NRS. Accordingly, participants with a history of ACE showed a less rapid and weaker decline of EMG over the course of the experiment compared to participants without ACE. In addition, participants with a history of ACE on average gave higher NRS scores, which, over the course of the experiment, scores decreased less compared to the scores of people without a history of ACE.

Taken together, the findings of the present study support the hypothesis that childhood adversity can lead to heightened stress sensitivity, which can for instance manifest at the physiological level as increased EMG stress-reactivity at the when exposed to a (nontraumatic) stressor. Translating this to daily life, this could mean that people who have been exposed to early life stress are more likely to experience a state of physiological hyperarousal in adulthood when confronted with a stressor. This stressor, importantly, does not need to be traumatic to elicit an increased stress-response; it may also be 
relatively mild. Arguably, the earlier and more (toxic) stress one has been exposed to, the more sensitive one becomes to daily life hassles. Hence, the stronger effect for early ACE. Additionally, our findings also indicate that childhood adversity impedes habituation to pain. Given the large body of research demonstrating a relationship between childhood adversity and (chronic) pain conditions, this observation may be of interest with respect to underlying mechanisms.

\section{Implications}

Findings of the present study emphasize the fact that childhood adversity does not only impact the brain and mind, but also the body. Accordingly, as shown in this and previous papers, the long-lasting influence of childhood adversity can be examined on a (psycho) physiological level by measuring trapezius muscle stress reactivity. The observation that exposure to ACE results in increased EMG stress-reactivity when confronted with a (physical) stressor, and, moreover, in diminished habituation to the stressor, is thought to be meaningful with respect to both mental and physical health problems, and the treatment thereof.

First, it highlights the notion that people with a history of ACE may be more sensitive to stress, thus being at increased risk to experience a state of physiological hyperarousal when faced with daily life stress, let alone significant life events. Since being in a state of physiological hyperarousal too long and/or too frequent is known to severely negatively affect both mind and body, arguably, this may point at a mechanism by which childhood adversity causes health problems on the long run.

Second, the observations in this study stress the importance of (also) addressing the body when treating stress and/or trauma-related issues. Issues that, importantly, can come in many shapes and forms (e.g., addiction, psychosis and/or chronic pain), and thus require specific enquiry of childhood adversity to be able to understand as such. ${ }^{49}$ Given increased stress sensitivity and physiological hyperarousal as a consequence of childhood adversity, it can be argued that learning skills to self-regulate one's physiology should be a main treatment goal for people with a history of ACE. Importantly, this likely cannot be done by only using cognitive interventions. For future research and clinical practice, it is thus pivotal to explore and implement new ways of also addressing the body. ${ }^{50}$

With respect to underlying mechanisms leading to chronic pain, the observation that a history of ACE impedes habituation to pain, is thought to be of interest. Although a substantial body of research demonstrated a relationship between childhood adversity and (chronic) pain conditions, mechanistic evidence remains 
to be elucidated. Perhaps, as suggested in the present study, increased sensitivity and reactivity to stress (i.e., pain) as a result of early life adversity, is a pathway that may lead to a whole range of pain-related issues later in life, since it can make people more vulnerable to the negative impact of (daily life) stress faced in adulthood. Arguably, depending on other factors, this impact may increase the risk for developing mental health conditions in some, and pain conditions in others. Replication of the present study for specific pain populations is thought to be of interest in order to further elucidate the relationship between childhood adversity and chronic pain.

Finally, as for mental health conditions, enquiring about childhood adversity is thought to be pivotal with respect to addressing and treating (chronic) pain. Especially when a patient with pain has a significant history of trauma, next to a merely medical treatment, additional psychological treatment (that also addresses the body) may prove to be effective for treating and managing pain symptoms. ${ }^{51}$

\section{Limitations of the study}

Results of the present study should be viewed in the light of several limitations. First, ACE were measured with a self-report questionnaire that is known to be prone to recall and response bias. It is difficult to perceive, however, how these would directionally affect physiological outcomes. Additionally, the questionnaire used measured ACE at a general level; it only assessed whether an event took place or not. For future research, it is recommended to explore ACE in more detail by also collecting information about timing, frequency and impact. Notably, despite these limitations, this study did yield significant results.

Second, EMG was measured with only two electrodes placed on the left and right trapezius muscle. For future studies, we suggest using more electrodes and/ or attaching electrodes to other body parts to enhance reliability of the EMG measurements. This might also be effective to further explore the discrepancy between results for left and right trapezius muscle reactivity. Interestingly, a left-right discrepancy in the similar direction was also observed by Luijcks and Marsman, 32,37 suggesting there may be an underlying mechanism that requires further elucidation. Since the painful stimulus was administered to the left middle finger, we had hypothesized that this would contribute to at least some of the discrepancy between the left and right trapezius muscle activity, yet it may not fully explain the different results. 
Finally, this study assessed a sample of the general population, in which the distribution of both ACE and current (chronic) pain conditions was relatively limited. Replicating this study in a clinical population may yield more pronounced results and is thus desirable. Despite these limitations, however, our analyses did shed light on the relationship between childhood adversity, stress sensitivity and habituation to pain.

\section{Conclusion}

In conclusion, this study provided support for the hypothesis that childhood adversity may impact later stress sensitivity, resulting in greater EMG reactivity and diminished habituation when exposed to a physical stressor.

\section{Acknowledgments}

We want to thank Jacco Ronner for designing the memory task (department of psychology, Maastricht University) and Ron Mengelers for providing technical support (department of psychiatry and neuropsychology, Maastricht University). 


\section{REFERENCES}

1. Felitti VJ, Anda RF, Nordenberg D, Williamson DF, Spitz AM, Edwards V, Koss MP, Marks JS. Relationship of childhood abuse and household dysfunction to many of the leading causes of death in adults. The Adverse Childhood Experiences (ACE) Study. Am J Prev Med May 1998;14(4):245-258.

2. Asmundson G. Adverse childhood experiences : using evidence to advance research, practice, policy, and prevention. 1. ed. San Deigo: Elsevier; 2019.

3. McLaughlin KA, Greif Green J, Gruber MJ, Sampson NA, Zaslavsky AM, Kessler RC. Childhood adversities and first onset of psychiatric disorders in a national sample of US adolescents. Arch Gen Psychiatry Nov 2012;69(11):1151-1160.

4. Dong M, Anda RF, Felitti VJ, Dube SR, Williamson DF, Thompson TJ, Loo CM, Giles WH. The interrelatedness of multiple forms of childhood abuse, neglect, and household dysfunction. Child Abuse Negl Jul 2004;28(7):771-784.

5. Van der Kolk BA. The body keeps the score : brain, mind, and body in the healing of trauma. New York: Viking; 2014.

6. Merrick MT, Ford DC, Ports KA, Guinn AS. Prevalence of Adverse Childhood Experiences From the 2011-2014 Behavioral Risk Factor Surveillance System in 23 States. JAMA Pediatr Nov 1 2018:172(11):1038-1044.

7. Zhang L, Mersky JP, Topitzes J. Adverse childhood experiences and psychological well- being in a rural sample of Chinese young adults. Child Abuse Negl Oct 2020;108:104658.

8. Service C-CRaDI. Final Report Summary - BECAN (Balkan Epidemiological Study on Child Abuse and Neglect) 2015.

9. Veleminsky M, Sr., Dvorackova O, Samkova J, Rost M, Sethi D, Veleminsky M, Jr. Prevalence of adverse childhood experiences (ACE) in the Czech Republic. Child Abuse Negl Apr 2020;102:104249.

10. Vink RM, van Dommelen P, van der Pal SM, et al. Self-reported adverse childhood experiences and quality of life among children in the two last grades of Dutch elementary education. Child Abuse \& Neglect 2019/09/01/ 2019;95:104051.

11. Anda RF, Felitti VJ, Bremner JD, Walker JD, Whitfield C, Perry BD, Dube SR, Giles WH. The enduring effects of abuse and related adverse experiences in childhood. A convergence of evidence from neurobiology and epidemiology. Eur Arch Psychiatry Clin Neurosci Apr 2006;256(3):174-186.

12. Bremner JD. Long-term effects of childhood abuse on brain and neurobiology. Child Adolesc Psychiatr Clin N Am Apr 2003:12(2):271-292.

13. Danese A, McEwen BS. Adverse childhood experiences, allostasis, allostatic load, and age-related disease. Physiol Behav Apr 12 2012:106(1):29-39.

14. De Bellis MD, Zisk A. The biological effects of childhood trauma. Child Adolesc Psychiatr Clin N Am Apr 2014;23(2):185-222, vii.

15. Solmi M, Radua J, Stubbs B, et al. Risk factors for eating disorders: an umbrella review of published meta-analyses. Braz J Psychiatry Sep 282020.

16. Cheong EV, Sinnott C, Dahly D, Kearney PM. Adverse childhood experiences (ACEs) and laterlife depression: perceived social support as a potential protective factor. BMJ Open Sep 1 2017;7(9):e013228.

17. Kendler KS, Kuhn JW, Prescott CA. Childhood sexual abuse, stressful life events and risk for major depression in women. Psychol Med Nov 2004;34(8):1475-1482

18. Lardinois M, Lataster T, Mengelers R, Van Os J, Myin-Germeys I. Childhood trauma and increased stress sensitivity in psychosis. Acta Psychiatr Scand Jan 2011;123(1):28-35. 
19. Edwards VJ, Holden GW, Felitti VJ, Anda RF. Relationship between multiple forms of childhood maltreatment and adult mental health in community respondents: results from the adverse childhood experiences study. Am J Psychiatry Aug 2003;160(8):1453-1460.

20. Dube SR, Felitti VJ, Dong M, Chapman DP, Giles WH, Anda RF. Childhood abuse, neglect, and household dysfunction and the risk of illicit drug use: the adverse childhood experiences study. Pediatrics Mar 2003:111(3):564-572.

21. Nelson CA, Bhutta ZA, Burke Harris N, Danese A, Samara M. Adversity in childhood is linked to mental and physical health throughout life. BMJ 2020:m3048.

22. Felitti VJ. [The relationship of adverse childhood experiences to adult health: Turning gold into lead]. Z Psychosom Med Psychother 2002;48(4):359-369.

23. Brown DW, Anda RF, Tiemeier H, Felitti VJ, Edwards VJ, Croft JB, Giles WH. Adverse childhood experiences and the risk of premature mortality. Am J Prev Med Nov 2009;37(5):389-396.

24. Shonkoff JP, Garner AS, Committee on Psychosocial Aspects of C, Family H, Committee on Early Childhood A, Dependent C, Section on D, Behavioral P. The lifelong effects of early childhood adversity and toxic stress. Pediatrics Jan 2012;129(1):e232-246.

25. Ridout KK, Khan M, Ridout SJ. Adverse Childhood Experiences Run Deep: Toxic Early Life Stress, Telomeres, and Mitochondrial DNA Copy Number, the Biological Markers of Cumulative Stress. Bioessays Sep 2018;40(9):e1800077.

26. Read J, Perry BD, Moskowitz A, Connolly J. The contribution of early traumatic events to schizophrenia in some patients: a traumagenic neurodevelopmental model. Psychiatry Winter 2001;64(4):319-345.

27. Solomon EP, Heide KM. The biology of trauma: implications for treatment. J Interpers Violence Jan 2005;20(1):51-60.

28. Lataster J, Myin-Germeys I, Lieb R, Wittchen HU, van Os J. Adversity and psychosis: a 10- year prospective study investigating synergism between early and recent adversity in psychosis. Acta Psychiatrica Scandinavica May 2012:125(5):388-399.

29. Glaser JP, van Os J, Portegijs PJ, Myin-Germeys I. Childhood trauma and emotional reactivity to daily life stress in adult frequent attenders of general practitioners. J Psychosom Res Aug 2006;61(2):229-236.

30. Wichers M, Schrijvers D, Geschwind N, et al. Mechanisms of gene-environment interactions in depression: evidence that genes potentiate multiple sources of adversity. Psychol Med Jul 2009;39(7):1077-1086.

31. Rauschenberg C, van Os J, Cremers D, Goedhart M, Schieveld JNM, Reininghaus U. Stress sensitivity as a putative mechanism linking childhood trauma and psychopathology in youth's daily life. Acta Psychiatr Scand Oct 2017;136(4):373-388.

32. Marsman A, Luijcks R, Vossen C, van Os J, Lousberg R. The impact of adverse childhood experiences on EMG reactivity: A proof of concept study. PLoS One 2019;14(5):e0216657.

33. Luijcks R, Vossen CJ, Roggeveen S, van Os J, Hermens HJ, Lousberg R. Impact of early life adversity on EMG stress reactivity of the trapezius muscle. Medicine (Baltimore) Sep 2016;95(39):e4745.

34. Collip D, Myin-Germeys I, Van Os J. Does the concept of "sensitization" provide a plausible mechanism for the putative link between the environment and schizophrenia? Schizophr Bull Mar 2008;34(2):220-225.

35. Davis DA, Luecken LJ, Zautra AJ. Are reports of childhood abuse related to the experience of chronic pain in adulthood? A meta-analytic review of the literature. Clin J Pain Sep-Oct 2005;21(5):398405. 
36. Sachs-Ericsson N, Blazer D, Plant EA, Arnow B. Childhood sexual and physical abuse and the 1-year prevalence of medical problems in the National Comorbidity Survey. Health Psychol Jan 2005;24(1):32-40.

37. Luijcks R, Hermens HJ, Bodar L, Vossen CJ, Van Os J, Lousberg R. Experimentally induced stress validated by EMG activity. PLoS One 2014;9(4):e95215.

38. Wijsman J, Grundlehner B, Penders J, Hermens H. Trapezius Muscle EMG as Predictor of Mental Stress. Vol 12; 2010.

39. Thompson RF, Spencer WA. Habituation: a model phenomenon for the study of neuronal substrates of behavior. Psychol Rev Jan 1966;73(1):16-43.

40. Reich CG, Taylor ME, McCarthy MM. Differential effects of chronic unpredictable stress on hippocampal CB1 receptors in male and female rats. Behav Brain Res Nov 5 2009;203(2):264-269.

41. Vossen CJ, Luijcks R, van Os J, Joosten EA, Lousberg R. Does pain hypervigilance further impact the lack of habituation to pain in individuals with chronic pain? A cross-sectional pain ERP study. J Pain Res 2018:11:395-405.

42. Bromm B, Lorenz J. Neurophysiological evaluation of pain. Electroencephalogr Clin Neurophysiol Oct 1998;107(4):227-253.

43. van Os J, Rutten BP, Poulton R. Gene-environment interactions in schizophrenia: review of epidemiological findings and future directions. Schizophr Bull Nov 2008;34(6):1066-1082.

44. Costa PT, Jr., McCrae RR. Stability and change in personality assessment: the revised NEO Personality Inventory in the year 2000. J Pers Assess Feb 1997;68(1):86-94.

45. McCrae RR. The five-factor model and its assessment in clinical settings. J Pers Assess Dec 1991;57(3):399-314.

46. Spielberger CD, Gorsuch, R.L., Lushene, R., Vagg, P.R. and Jacobs, G.A. Manual for the State-Trait Anxiety Inventory. Palo Alto: Consulting Psychologists Press; 1983.

47. Cohen S, Kamarck T, Mermelstein R. A global measure of perceived stress. J Health Soc Behav Dec 1983;24(4):385-396.

48. Noone P. The Holmes-Rahe Stress Inventory. Occup Med-Oxford Oct 2017;67(7):581- 582.

49. Pearce J, Murray C, Larkin W. Childhood adversity and trauma: experiences of professionals trained to routinely enquire about childhood adversity. Heliyon 07/01 2019;5:e01900.

50. van der Kolk B. Posttraumatic stress disorder and the nature of trauma. Dialogues Clin Neurosci Mar 2000;2(1):7-22

51. Clay Jackson W. Connecting the Dots: How Adverse Childhood Experiences Predispose to Chronic Pain. Practical Pain Management. 18 June 2020. Available at: https://www.practicalpainmanagement. com/treatments/psychological/connecting-dots-how-adverse-childhood-experiences-predisposechronic-pain. Accessed 13 April 2021. 



\section{CHAPTER 6}

GENERAL DISCUSSION 


\section{GENERAL DISCUSSION}

The central aim of this dissertation was to investigate underlying mechanisms that may contribute to the emergence of mental illness, specifically focusing on the trajectory from childhood adversity to mental ill-health later in life. For this purpose, we explored the contribution of both genetic and environmental factors, emphasizing the role of childhood adversity by examining its long-lasting impact at the cognitive, psychological, and psychophysiological level, as well as at the epidemiological level of transition from health to ill-health. In the following paragraphs of this discussion, main findings of the previous chapters are reviewed briefly, followed by more in-depth considerations and, finally, future recommendations for research and clinical practice with respect to childhood adversity in relation to mental health. 


\section{MAIN FINDINGS}

\section{Genes and environment in relation to mental health}

Based on the findings of numerous twin and family studies and recent advances in neuroscience, it has become clear that genetic and environmental factors do not act in isolation Accordingly, it is thought that neither genes nor environmental exposure(s) can independently account for the causation of mental illness, but most likely contribute jointly and interactively. While both factors are considered important in terms of contribution to the ontogenesis of psychopathology and subsequent mental disorders, little is known on how these factors contribute to variance in mental health, and to what extent. In this dissertation (chapter $\mathbf{2}$ ), we studied this issue in a large population-based cohort by analyzing the impact of modern molecular measures of genetic risk (i.e., polygenic risk score, PRS) and measures of environmental risk (including childhood adversity) at the level of and change in general mental health.

Confirming with previous research, we predicted that familial (genetic and non-genetic) and environmental risk factors would outperform the contribution of genetic factors at the molecular genetic level, which was indeed demonstrated. We calculated the relative contribution of each (group of) risk factor(s) to the variance in mental health and found that in the combined model (including all molecular genetic and environmental factors) around $17 \%$ of the variance in mental health could be explained by familial and environmental factors, while only $0.4 \%$ could be explained by PRS. Hence, socio-environmental factors accounted for the bulk of the explained variance, particularly childhood adversity and family history - the latter probably representing environmental influences to a large degree. With respect to change in mental health status over time, familial and environmental factors explained around $12 \%$ of the variance, PRS on the other hand, did not significantly contribute. Again, childhood adversity had the largest impact, followed by recent life events.

Despite the considerable gap between the contribution of molecular genetic and environmental factors, results of our study cannot be interpreted as showing that genetic factors are unimportant. They most likely are, but - as research is continuously demonstrating - when it comes to mental health, genetic factors do not act in isolation, but in interaction with the environment. Hence, the impact of genetic factors may be best captured by gene-environment interactions and not by isolated main effects.

\section{The impact of childhood adversity on cognitive functioning}

To date, it has been well established that exposure to stressful and traumatic circumstances early in life can result in long-term impairments at all levels of human functioning, including cognitive abilities. Indeed, there is considerable research 
demonstrating the detrimental impact of childhood adversity on cognitive functioning throughout later life. ${ }^{2,3}$ However, findings have been inconsistent and were often based on studies with a relatively small sample size. ${ }^{4,5}$ In order to further elucidate the relationship between childhood adversity and cognition, we investigated this in a longitudinal study that included people with psychotic disorder, their siblings and healthy comparison subjects (chapter $\mathbf{3}$ ). With respect to people with psychotic disorder, it was of specific interest to study the interplay between childhood adversity and cognition, given that both a history of childhood adversity and cognitive impairments are highly prevalent in this population. ${ }^{5}$ We furthermore took several known issues into account, such as adequate sample size and repeated measures of the cognitive outcome for added stability and precision of measurement (i.e., IQ).

With respect to the impact of childhood adversity on $I Q$, we found significant differences across the 3 groups. In healthy controls, exposure to childhood adversity was associated with a nearly 5-point reduction in IQ. A smaller reduction, at 2.5, was found in siblings, while for people with psychotic disorder no significant reduction could be demonstrated. These findings suggest that the impact of childhood adversity on cognitive abilities differs between individuals who are touched by psychosis directly (e.g., patients themselves), indirectly (e.g., siblings of patients) and individuals who are not affected (e.g., healthy controls). Around $25 \%$ of the difference between siblings and healthy controls was attributable to childhood adversity, compared to only $5 \%$ of the patient-control comparison difference. An explanation for this finding, as offered in chapter $\mathbf{3}$, might be that the cognitive alterations observed in people with a psychotic disorder are not, or only minimally, influenced by childhood adversity, but attributable to other factors underlying psychotic symptoms such as genetic effects, the effects of recent life events and/or social isolation. In addition, it is suggested that people suffering from psychosis may also have altered motivation to engage in cognitive assessments, which might further explain the differential findings. ${ }^{6}$ Taken together, the absence of an association between childhood adversity and cognition in the patient group may be due to a 'floor effect', meaning that the impact of childhood adversity is not non-existent, but possibly 'outperformed' by other factors.

With respect to the relationship between childhood adversity and learning abilities, we found that childhood adversity impacted on the course of IQ over time, in the sense of showing less learning effects of repeated cognitive assessments over time. In contrast to the previous finding, this effect was found regardless of group. The fact that we did find a significant association regarding learning abilities in the patient group as well, suggests that childhood adversity is not 'cognition-neutral' in the patient group, thus supporting the notion of a floor effect due to other, possibly competing or confounding, factors. 
To summarize, our findings suggest that childhood adversity impacts cognitive abilities and impedes learning. Its impact on observed cognitive alterations in people with a psychotic disorder (and possibly other mental disorders as well), however, may be relatively small given the likelihood that other factors impacting on cognitive abilities compete with the effect of childhood adversity in this particular group.

\section{The impact of childhood adversity on EMG stress reactivity}

There is growing evidence that exposure to childhood adversity increases stress sensitivity later in life, which in turn is thought to increase the risk of developing a mental disorder following adult stressful life events. ${ }^{7.8}$ Although experiences of childhood adversity can be highly variable, there are several common features to be recognized that might explain part of the underlying mechanism(s) by which early adversity can cause long-lasting impairments. Foremost among those is the fact that adverse childhood experiences by nature are stressful and stress inducing. Additionally, as pointed out in chapter $\mathbf{4}$, they often involve a high degree of unpredictability. Based on human and animal research, it is suggested that during childhood, the unpredictability of an event (e.g., not knowing when, why or from where a stressor may arise) may be most impactful, especially for younger children. For instance, animal research on chronic unpredictable stress supports the notion that the degree of unpredictability mediates its harmful consequences, showing that completely predictable stress, even if more severe, has less effect on certain brain areas compared to moderate unpredictable stress. ${ }^{9}$

Stress per definition causes physiological changes, ${ }_{1}^{10}$ such as in heart rate, cortisol levels and muscle tension levels, and these changes may vary between hardly noticeable to extreme. With respect to muscle tension, previous research has shown that trapezius muscle stress reactivity is a good candidate for examining the impact of childhood adversity on a physiological stress-related outcome. In a study by Luijcks and co-workers it was found that anticipatory electromyography (EMG) stress reactivity in a stress experiment using a single unpredictable electrical painful stimulus, was consistently stronger in participants with higher levels of ACE.112

In this dissertation, we set out to further explore the relationship between childhood adversity and (increased) stress sensitivity, extending the findings of Luijcks and colleagues on the influence of childhood adversity on EMG activity, by conducting two different stress experiments. The first was a memory task using a cognitive stressor (chapter 4), and a habituation task using a series of electrical painful stimuli (chapter 5). In order to investigate the role of predictability in the experiment, as was also assessed by Luijcks, the study protocol of both tasks included an identical session six months after the first session. 
Since we used two experimental stress paradigms that, to our knowledge, have not been used before, a first objective in both studies was to validate the stress paradigm by investigating a condition effect, using a baseline measurement, during which participants sat still with their eyes open, as the 'control' condition. As expected, for both the memory and the habituation task, EMG stress reactivity was significantly higher compared to the baseline condition. Hence, both tasks could be considered stress inducing. In accordance with our hypothesis regarding the role of predictability, a significant session effect was found (i.e., decreased EMG reactivity during the second session compared to the first), likely due to a reduction in unpredictability during the second session.

In line with previous research, in both the memory and habituation task, childhood adversity was associated with increased EMG stress reactivity. We furthermore found evidence for a dose-response relationship, demonstrating that participants with higher ACE scores showed increased EMG reactivity relative to those with lower ACE scores. Translating these findings to daily life situations, it is conceivable that individuals who have experienced childhood adversity may react with stronger muscle tension when confronted with an unpredictable and/or uncertain event, relative to those who did not experience early adversity.

Taken together, both studies offer support for the hypothesis that childhood adversity may result in heightened sensitivity to stress, that persists throughout life, measurable on the psychophysiological level of EMG stress reactivity.

\section{The impact of childhood adversity on habituation to pain}

Pain, and chronic pain more specifically, is a highly prevalent, complex, and distressing problem that can have profound impact on one's quality of life. According to the Global Burden of Disease reviews ${ }^{13,14}$, pain affects between one-third and one-half of the population, thus representing one of the most prominent causes of disability worldwide. As mentioned earlier, childhood adversity has been identified as a risk factor for developing a wide range of mental and physical problems, including numerous pain-related conditions. The experience of (bodily) pain is furthermore strongly associated with mental ill-health, and ill-health in general, as was also observed in our study described in chapter 2. Accordingly, there is a substantial body of research demonstrating a relationship between childhood adversity, (chronic) pain and mental illness. However, relatively little is known about the direction of these associations and underlying (biopsychosocial) mechanisms. ${ }^{15-17}$ 
In this dissertation (chapter 5), we addressed this issue by conducting a stressexperiment (i.e., habituation task) that allowed us to explore the relationship between childhood adversity, stress sensitivity and habituation to pain. As mentioned above, during the habituation task a series of 25 painful electrical stimuli were administered to the left middle finger, while measuring EMG activity. In order to ensure that the stimulus was indeed perceived as painful, hence, representing pain, for each participant, the individual sensation and pain threshold was determined and used to calculate a stimulus intensity level that was 25\% above the pain threshold. During the experiment, both the intensity and number of stimuli were unknown to the participant. While identical, participants were asked to verbally rate the intensity of each stimulus, thereby enabling additional analyses of subjective pain report, next to analyzing EMG.

Since we used a stress-protocol that, to our knowledge, has not been used before in combination with EMG, next to validating the experiment as stress-inducing (described above), we also wished to validate the experiment as measuring a form of habituation. Indeed, both a within-session and between-session habituation effect was observed: EMG decreased in the inter-stimulus-intervals as well as over the course of 25 stimuli. Furthermore, EMG activity was attenuated during the second session. These findings were in accordance with the findings described in chapter 4, as well as previous research on EMG activity.112 In addition, we also observed a habituation effect at the level of pain report, finding decreased numeric rating scores (NRS) for each consecutive stimulus. As hypothesized a priori, this effect did not differ between sessions.

Besides validating the habituation task as a valid stress-experiment that measures a form of habituation, the primary objectives of our study were to investigate the relationship between childhood adversity and EMG stress reactivity (reviewed above) and, moreover, between childhood adversity and habituation to pain. With respect to the latter, results indicated that higher ACE-scores are associated with diminished habituation, both in the short term (i.e., at the inter-stimulus-interval level) as well as in the longer term (i.e., at the stimulus number and session level) and, thus, moderating a within-session and a between-session habituation effect. Next, we found that NRS scores were also significantly moderated by childhood adversity, in such a way that participants with a history of ACE, on average, reported higher NRS scores relative to those without a history of ACE. In addition, over the course of 25 stimuli, scores of people with ACE did not decrease as much as for the non-ACE group. 
In sum, results of this study indicate a moderating effect of childhood adversity not only on EMG stress reactivity in general, but also on habituation to pain more specifically: higher ACE scores were associated with increased EMG reactivity and diminished habituation, both at the objective level of EMG as well as at the subjective level of pain report. 


\section{IN-DEPTH CONSIDERATIONS}

Based on the above, the conclusion can be drawn that this dissertation provides support for the notion that childhood adversity causes lasting impact on (arguably all) dimensions related to health and well-being. This impact was shown to be measurable in the general population, and, furthermore, to potentially influence an individual at the level of everyday life, given evidence for increased stress reactivity when faced with a stressor, diminished habituation to pain and impaired learning abilities.

In the following paragraphs, several in-depth considerations concerning childhood adversity in general, and the findings of this dissertation more specifically, are outlined. Additionally, I propose a model of the trajectory from having experienced adversity early in life, to the onset of mental illness in adulthood. In this model, childhood adversity is considered a transdiagnostic mediating and moderating factor.

\section{Adversity or Trauma?}

In this dissertation, I explicitly used the term 'adversity' rather than 'trauma' to refer to stressful and (potentially) harmful experiences, on the one hand attempting to stay away from the discussion of what is to be considered trauma, and what not, and, on the other hand, aiming to primarily focus on the aspect of 'stressfulness' related to an event or experience, and the impact thereof. Adversity, in my opinion, best captures this aspect and is furthermore considered to resemble a broader perspective as opposed to the concept of trauma, which appears to be narrower. It should, however, be noted that, although both concepts are widely used in the scientific literature, consensus on their definitions and so-called conceptual brackets is lacking. Several interesting articles have been published addressing this issue, for instance by McLaughlin ${ }^{18}$ and Krupnik ${ }^{19}$, and while for this dissertation it would be beyond the purported scope to extensively elaborate on the issue, several issues are considered worth pointing out.

First, a major limitation regarding the construct of trauma, more specifically, the way it is defined and used within diagnostical systems such as the DSM, is the fact that inherent to its very definition, is the failure to acknowledge certain events and/or experiences as traumatic, while they most certainly can be. To exemplify: the first (A) criterion of post-traumatic stress disorder (PTSD), as described in the DSM-V, describes a traumatic event as follows: "The person was exposed to: death, threatened death, actual or threatened serious injury, or actual or threatened sexual violence, in the following way(s): direct exposure; witnessing the trauma; learning that a relative or close friend was exposed to a trauma; indirect exposure to aversive details of the trauma, usually in the course of professional duties (e.g., first responders, medics).20 
This so-called A criterion needs to be met, otherwise PTSD cannot be diagnosed, even though all other criteria may be met and the event, or events, causing subsequent suffering are clearly to be considered adverse.

One could argue that not receiving a certain diagnosis might not be a serious problem, however, when, as a consequence, you are denied treatment for symptoms clearly belonging to the very diagnosis you are not allowed, it most definitely is a serious problem. Importantly, this is not hypothetical, this is reality. It affects countless people still suffering from the legacy of neglect and deprivation they have been exposed to in their childhoods.

Second, with respect to PTSD and the A-criterion trauma, it is important to note that adverse events or experiences failing to meet the typical definitions of trauma (e.g., emotional neglect), nonetheless contribute to predicting the onset of a wide range of mental disorders. Indeed, in childhood, physical and emotional neglect can be at least as harmful as sexual, physical, and emotional abuse. Moreover, there is evidence showing that the long-standing effects of emotional cruelty and neglect on a neurobiological level, as well as at the level of mental health, may be even more significant than those of physical abuse. 21,22

Hence, it is suggested to not hold on to relatively narrow definitions of trauma (such as described in the DSM-V) too tightly when assessing and/or addressing the impact of childhood adversity. In addition, in order to comprehend the impact at the level of the exposed individual, one should prioritize the subjective experience over the objective event, given the fact that personal reactions to aversive events are highly context-dependent. Furthermore, they are influenced by individual factors to a great extent, such as for instance the subjective experience of loss of control during and social support after the event.

Last, there are many questions to be raised with respect to both the concept of adversity, and that of trauma. For example, where do we draw the line between 'normal' experiences of stress, and those that qualify as adverse? Similarly, where does adversity end, and trauma begin? Some researchers propose a dimensional view of adversity, where trauma is considered the highest degree. ${ }^{23,24}$ While this approach seems attractive, it is also challenging to operationalize its constructs with respect to research and clinical practice, thus possibly making it less instrumental.

This exact problem has been pointed out by McLaughlin in relation to childhood adversity, ${ }^{18}$ arguing that parsing different types of adversity as well as trauma is considered valuable in order to elucidate specific links between adversity and the 
onset of mental disorders. So, to advance knowledge of mechanisms underlying the association between childhood adversity and mental illness, utilization of consistent definitions is highly recommended, moreover, thought to be pivotal.

Importantly, these definitions should not be too wide, thereby pathologizing 'normal' human suffering, but also not too narrow, excluding types or forms of adversity that from a subjective perspective can be, and have been, clearly traumatic.

\section{Assessing childhood adversity: towards a dimensional approach}

While there is little debate regarding the pervasive detrimental effects of childhood adversity on health and well-being, it is far less clear how and why early adversity exerts such a profound influence. Arguably, a key explanation for this fact concerns the approach and assessment of childhood adversity, having crucial implications for both research and clinical practice in this field.

For decades, most research on the impact of childhood adversity focused on single types of adversity, such as sexual abuse or living in poverty. As pointed out earlier, a critical limitation of this approach is that it fails to account for the fact that adverse events tend to co-occur. ${ }^{25}$ Subsequently, when assessing the impact of childhood adversity, adverse experiences should not be assumed to be stand-alone events, but rather as co-occurring with other types of adversity, thus representing a complex set of highly interrelated experiences.

Accordingly, when examining single types of adversity in relation to (for instance) mental health, it is virtually impossible to determine whether a particular outcome (e.g., depression or psychosis) is a consequence of the type of adversity examined (e.g., physical abuse), or of other, non-assessed, adversities possibly also experienced by the individual.

As it became clearer that ACEs often co-occur, the approach of childhood adversity gradually shifted. Nowadays, most of the existing research, including the studies conducted in this dissertation, rely on what can be termed the 'cumulative risk approach'. This approach tallies the number of adverse events (or experiences) to create a risk score, such as the widely used ACE-score ${ }^{26}$. To illustrate, having experienced physical abuse, domestic violence, and a parent suffering from addiction, would result in a risk score of three. Similarly, a combination of emotional neglect, maternal depression and living in poverty also results in a risk score of three. 
While this approach has advantages over the single-type approach, it also has important limitations given the fact that cumulative risk focuses on the number of distinct events, rather than on the severity or type of adversity. As a consequence, it may be implicitly assumed that a higher risk score is per definition 'worse' and more impactful than a lower score. This assumption, however, is highly tenuous, given the fact that regardless of number, the impact of adverse events can significantly differ depending on type, timing, severity, and frequency thereof.

Despite known limitations, the cumulative risk approach has been widely adopted and proven to be useful, for instance with respect to highlighting the public health importance of childhood adversity. Furthermore, valuable research has been conducted using risk scores, which greatly increased our knowledge and understanding of the long-lasting impact of childhood adversity in general. ${ }^{37}$ To a great extent, such research (including this dissertation) regards dysregulation of the normal stress response (e.g., heightened stress sensitivity or HPA-axis hyperactivity) resulting from exposure to early life stress, as a central mechanism explaining the pervasive consequences of adversity. Importantly, while this is undoubtedly one pathway linking childhood adversity with a wide range of mental and physical health problems, it is not the only pathway.

Indeed, there are inconsistent findings with respect to stress response dysregulation and (mental) health outcomes. ${ }^{18}$ While clearly involved in the onset of chronic physical health problems ${ }^{27}$ and, possibly, mental disorders such as psychotic disorder, ${ }^{28}$ stress response dysregulation does not explain many disturbances in social and cognitive functioning commonly observed in children and adults with a history of early adversity. 18,29 Hence, in addition to the frequently invoked stress-pathways, it is likely that other mechanisms are at play, differentially causing impairments. This concerns mechanisms that cannot be elucidated by examining the impact of childhood adversity using cumulative risk scores, as it does not allow to distinguish between distinct types of adversity.

One alternative approach is offered by McLaughlin and Sheridan, attempting to distil complex adverse experiences into core underlying dimensions that cut across multiple forms of adversity. ${ }^{18}$ More specifically, their model differentiates between experiences of threat - involving harm or threat of harm, and deprivation - involving an absence of expected inputs from the environment (e.g., neglect and/or poverty). Further, rather than counting the number of adverse experiences, their approach assesses the frequency and severity of experiences reflecting each dimension and examines them simultaneously in predicting outcomes. Hence, this approach allows for the identification of mechanisms specifically linked to certain dimensions of adversity, as well as determining whether such mechanisms vary in relation to exposure-severity. 
With respect to the studies presented in this dissertation, it should be acknowledged that the assessment of childhood adversity has been compromised by several limitations. However, this does not imply that our findings lack meaning. To the contrary, in fact. Despite the known limitations, we observed significant results, demonstrating the long-lasting impact of childhood adversity at the cognitive, psychological, and psychophysiological level.

Replication of our findings, preferably examining a clinical population and using a more accurate form of assessing childhood adversity, such as proposed by McLaughlin and Sheridan, is desirable and thought to elicit more pronounced results that next to general underlying mechanisms, may help to elucidate more specific mechanisms with respect to different types of adversity, as well as its timing and frequency.

\section{Embracing heterogeneity}

In the introduction of this dissertation, several general principles with respect to the role of environmental factors within the biopsychosocial model are described. The results of the study on the contribution of genetic and environmental factors to the variance in mental health (chapter $\mathbf{2}$ ), are in accordance with these principles. Indeed, childhood adversity, representing an environmental factor, was shown to significantly contribute to the variance in level of mental health. Not with respect to a specific mental disorder, but mental health in general, thus possibly contributing to variance in many different conditions construed as mental disorders.

Notably, PRS-SZ (i.e., polygenic risk score for schizophrenia), representing a molecular genetic factor, was also found to significantly contribute, yet to a far less extent. As already outlined above, this result cannot be interpreted as showing that genetic factors are unimportant with respect to mental health. Rather, our results support the notion that the impact of genetic factors may be best captured by gene-environment interactions, and not by isolated main effects. This, in fact, may be equally true with respect to environmental factors such as childhood adversity, given that from a biopsychosocial perspective, different factors are thought to interact within and between dimensions.

To exemplify the complex interplay of factors between and within biopsychosocial dimensions, in figure 1 I have illustrated the possible different effects and their directions. They include within-dimensional main and interaction effects (e.g., age effecting physical health), bi-dimensional main and interaction effects (e.g., physical health effecting self-esteem), and finally multidimensional main and interaction effects (e.g., childhood adversity effecting neurochemistry, cognition, and social relationships). 


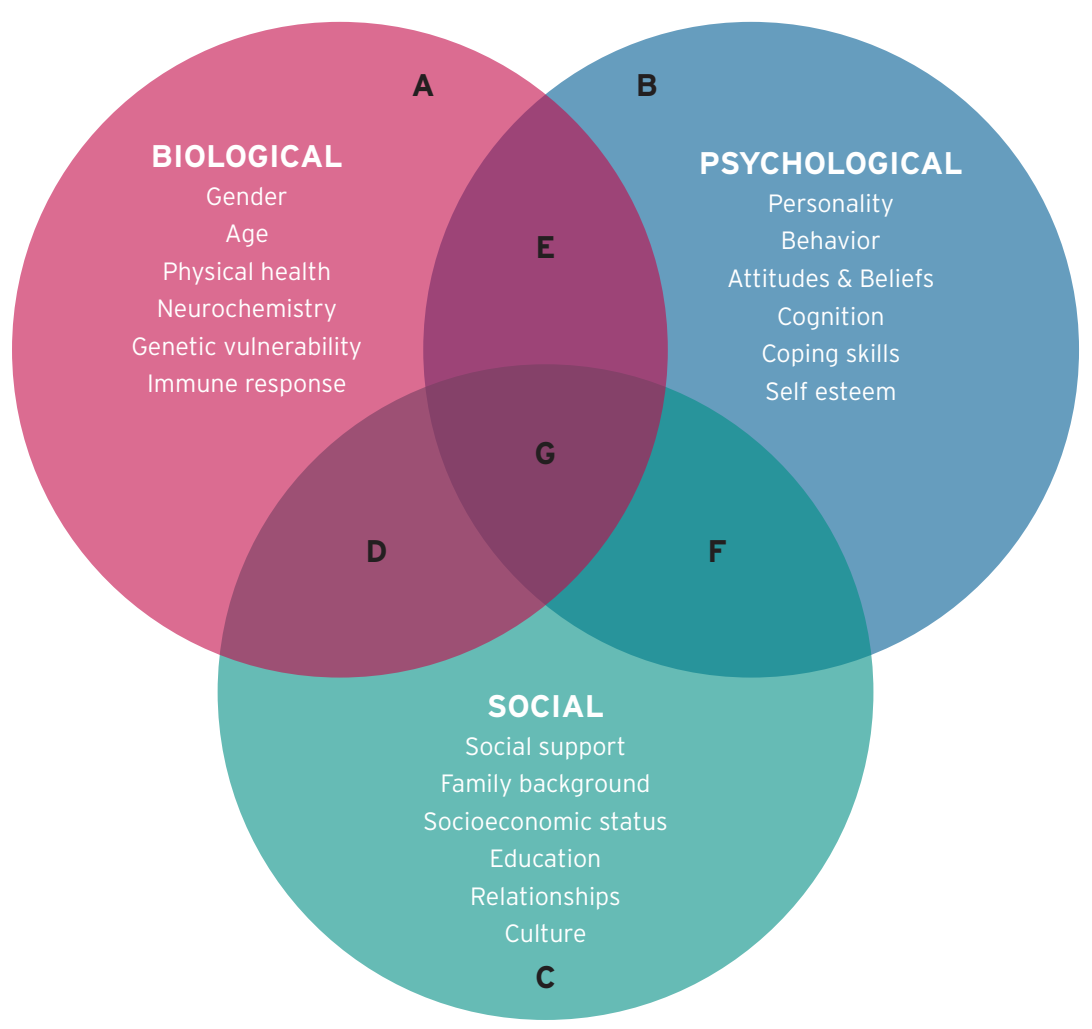

Figure 1. Illustration of the complex interplay between and within biopsychosocial dimensions Note. AB,C, within-dimensional main and interaction effects.

D.E.F bi-dimensional main and interaction effects.

${ }^{6}$ multidimensional main and interaction effects.

While the study of epigenetics and molecular genetics is still in its infancy, several findings regarding gene-environment interactions are important to consider. First, it has been shown that thousands of common and, less frequently, rare genetic variants contribute transdiagnostically to a wide range of mental disorders, thus demonstrating vast genetic overlap between conditions construed as disorders.' Second, the impact of environmental factors is likely conditional on genetic factors to a degree, resulting in gene-environment interactions. Additionally, the impact of the environment is also thought to depend on previous exposures, resulting in environment-environment interactions.' Third, most genetic and environmental factors that are known to influence (mental) health are non-specifically associated with a range of mental disorders. 
Indeed, there is growing evidence that multiple gene-environment interactions contribute transdiagnostically to most types of mental illness, but no specific constellation of gene-environment interactions explains a substantial proportion of cases. This might be explained by the fact that even if most risk factors are shared between two different mental disorders, the loading and combinations of factors that give rise to each of the two disorders may still be unique, thus demonstrating etiological heterogeneity to a degree.

To summarize, it is suggested that an individual case of mental illness is likely to be the result of multiple contributing biopsychosocial factors. Additionally, cases of the same mental illness in the general population are likely to be due to multiple etiological mechanisms. Hence, when it comes to mental (ill) health, heterogeneity is the rule, not the exception.

With respect to understanding and investigating underlying mechanisms that drive the emergence of mental illness, one should thus not only take into account genetic heterogeneity, but moreover etiological heterogeneity (i.e., poly-gene environmental interactions), and, possibly, also clinical heterogeneity, given the fact that one and the same mental disorder, as conceptualized by the DSM, can manifest itself in many different shapes and forms, overlapping with many if not most other mental disorders. Arguably, the heterogeneity observed within and between different categories of mental illness, may very well be the result of the complex interplay between genetic factors, environmental factors and subsequent gene-environment interactions involved in its etiology and transdiagnostic outcome.

\section{Bridging the gap: understanding the mind-body connection}

It can be argued that, within contemporary (mental) health care, the role of the body, and more specifically, the connection between the mind and the body, remains significantly overlooked and unaddressed. To date, cognitive-behavioral therapy (CBT) is still regarded the golden standard in the field of psychotherapy, primarily aimed at altering dysfunctional cognitions and behaviors that are thought to have caused and/or have contributed to symptomatology. One obvious explanation for this, is the simple fact that CBT is by far the most researched form of psychotherapy, thus having accumulated the most 'evidence' for its effectiveness. ${ }^{30}$ Consequently, CBT has been dominating the international guidelines for psychological treatments for decades, making it the 'evidence-based' treatment of choice for a wide range of mental disorders, as noted by the American Psychological Association. ${ }^{31}$ 
While it should be acknowledged that certain mental disorders can be characterized by dysfunctional cognitions and behaviors and CBT can be helpful in treating these, it should moreover be recognized that psychopathology may also be somatically driven. As explained by the biopsychosocial model, health and well-being, or the lack thereof, is the result of a complex interplay within and between somatic, psychological, and social dimensions. Although widely adopted, it can be argued that certain implications of the biopsychosocial model tend to be ignored, as is, arguably, the case with respect to interventions that fail to address the body in general, and the connection between the body and mind more specifically.

The notion that the mind (e.g., psychological and emotional factors) can affect the body (e.g., heart rate and muscle tension) is well established, as is the notion that mental health can (directly and indirectly) impact on physical health. ${ }^{32}$ Perhaps recognized to a lesser degree, yet equally true, is the fact that the body also affects the mind and, similarly, physical health is known to impact mental health. Indeed, mental disorders can have a dominant somatic cause, as has for instance been demonstrated by de Witte and colleagues, who provided an overview of numerous somatic disorders (e.g., vitamindeficiencies or diabetes) that can cause mood and psychotic symptoms. ${ }^{33}$ Accordingly, there is much evidence showing that mental health is influenced by neurobiological, immunological, endocrine and/or metabolic systems, more specifically, by dysregulation of these systems, and vice versa.

Existence of a close mind-body connection is furthermore demonstrated by the fact that inherently, mental health is expressed at the level of emotions and behavioral patterns, which are known to involve a strong physiological component. As pointed out by renowned neuroscientist Antonio Damasio, feelings are to be understood as mental experiences of body states - they arise as the brain interprets emotions, which are themselves purely physical signals of the body reacting to external stimuli. ${ }^{34}$ Feelings thus serve vital functions, such as signifying a physiological need (e.g., hunger), injury (e.g., pain), threat (e.g., fear or anger), or specific social interactions (e.g., love or compassion), thereby constituting a crucial component of preserving homeostasis, and, ultimately, life itself.

As a consequence, problems related to feelings, can have enormous impact with respect to one's health and well-being. Seen in this light, it may be argued that some of the most demanding health issues we face today, such as depression, chronic pain, and drug addiction, are first and foremost centered on what could be termed 'pathologies of feeling'. Arguably, addressing them as such might proof to be an effective strategy with respect to both prevention and treatment of such issues. 
In conclusion, the findings presented in this dissertation provide further evidence for the interplay between body and mind, moreover, supporting the notion that mental health impacts physical health, and vice versa. As will be argued in the next paragraph, childhood adversity may be considered both a transdiagnostic mediating and moderating factor, influencing the direct and indirect impact of mental health on physical health, as well as the other way around.

\section{Childhood adversity as a transdiagnostic mediating and moderating factor}

A conclusion that can be safely drawn, is that childhood adversity indeed can have longlasting impact. As demonstrated in the previous chapters, this impact is measurable in the general population, at the cognitive, psychological, and psychophysiological level. Consequently, it can be concluded that effects can be observed transdiagnostically in relation to the mind, as well as the body. Our results are furthermore supportive of the notion that childhood adversity does not directly impact health and well-being. In other words, it is no straight line, but involves an indirect, mediating and/or moderating effect. Hence, the impact of childhood adversity on adult health is thought to operate via various pathways that may be biological, ${ }^{35,36}$ psychological ${ }^{37}$ and/or social. ${ }^{38}$ While these pathways may operate independently, they may also be outcomes in and of themselves. Additionally, they may reinforce each other over time, all of which is in accordance with the biopsychosocial perspective on health.

Based on our findings and previous research, I propose two basic models to illustrate how childhood adversity might affect the relationship between mental and physical health in general, and the association between childhood adversity and mental health more specifically. Both models are based on the following principles:

1. Childhood adversity may impact on neurobiological, emotional and psychological development, resulting in impaired ability to self-regulate emotions, behaviors and nervous system activation;

2. Having been exposed to chronic and/or toxic stress in childhood may cause heightened sensitivity to stress and subsequent (chronic) physiological hyperarousal in adulthood;

3. 'Unhealthy behaviors' such as for instance eating too much or too little, selfharming and/or using drugs may first and foremost represent a coping-mechanism to self-regulate and deal with daily life stressors;

4. Having been exposed to chronic and/or toxic stress in childhood can inhibit a person's ability to make healthy decisions ${ }^{39}$, with respect to their physical health in the first place, but for instance also with respect to financial matters ${ }^{40}$, thereby predisposing people to unhealthy lifestyles and socioeconomic disadvantage. 
While, for the sake of simplification, the social dimension is left out in the conceptualization of the following models, the possible influence of this dimension should not be ignored. Further, these models should not be considered as competing, rather as complementing. They are meant to guide future research and clinical practice.

Childhood adversity in relation to mental and physical health

While a strong link between mental health and physical health has been extensively demonstrated, relatively little is known about the pathways from one to the other. Many mediating factors have been proposed and examined, lifestyle choices (e.g., smoking and physical activity) perhaps being the mediating factor for which the most evidence exists. ${ }^{32}$ With respect to childhood adversity, I propose that it can be considered both a mediating and a moderating factor, having the potential to influence the relationship between mental and physical health in divergent ways: either influencing one via the other (figure 2), or influencing the relation itself (figure 3). The direction and intensity of its influence is thought to be determined by the type of adversity, as well as aspects such as frequency and timing.

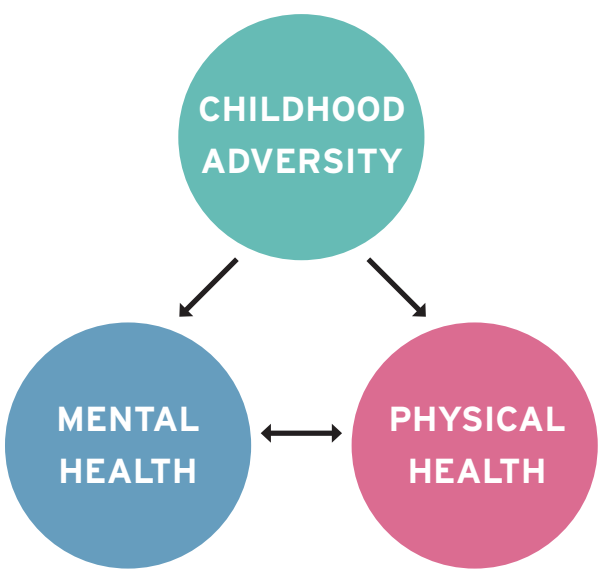

Figure 2. Childhood adversity as a mediator

Focusing on the relationship between childhood adversity and mental illness more specifically, I argue that the body, including all biological systems it embodies, is an underestimated factor in this relationship. Moreover, I argue that addressing the body should be considered a vital component with respect to preventing, as well as diagnosing and treating mental illness. 


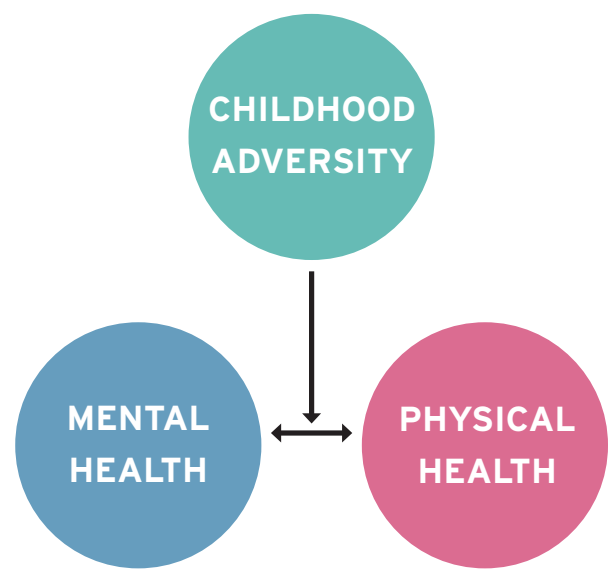

Figure 3. Childhood adversity as a moderator

Further, I propose that childhood adversity may directly impact the body, for instance by affecting one's neurobiology or immune system. In addition, it may also impact more general aspects related to the body, ${ }^{41,42}$ such as body-image, body-attitude, and body awareness. Body attitude, in this context, refers to cognitive, affective and behavioral aspects with respect to the body, ${ }_{1}^{43}$ while body awareness is defined as 'the perception of bodily states, processes and actions that is presumed to originate from sensory proprioceptive and interoceptive afferents and that an individual has the capacity to be aware of.'44

Taken together, what may have been some level of order and ease, may deteriorate into dis-order and dis-ease - or disorder and disease - as a consequence of having endured childhood adversity. 


\section{RECOMMENDATIONS}

Although the methodological considerations of the work in this dissertation have been discussed in the separate chapters, there are several overall limitations that should be considered for future research. In addition, based on the work presented here, several recommendations are provided with respect to clinical practice.

\section{Future research}

First, to advance knowledge of mechanisms underlying the association between childhood adversity and later health and well-being, utilization of consistent definitions and assessment tools is highly recommended. Moreover, thought to be essential for future research. In order to elucidate specific pathways underlying the trajectory from childhood adversity to later ill-health, assessment of adverse experiences should include key aspects such as type of adversity, timing, frequency and severity. Since findings suggest that it is not only the number of adverse events that is related to negative health outcomes, but moreover specific combinations of events (e.g., maternal depression and abuse), investigating different constellations of ACEs is furthermore thought to be important to enhance understanding of specific etiological mechanisms and their outcomes.

Second, the sample size of our studies that investigated EMG stress reactivity (chapter $\mathbf{4}$ and 5) were relatively small and included relatively little variance on several key between-subject variables such as childhood adversity and pain status, thus, regarded 'proof of principle' studies. Since one of the main objectives of both studies was to validate a newly designed stress-paradigm, the sample size was considered acceptable. Replication of the studies with bigger sample sizes is, obviously, desirable and thought to elicit more accurate results. Additionally, since we did not specifically assess a clinical population that is known to have experienced frequent and/or severe adversity, it is expected that stronger and more pronounced effects could be observed when examining a sample characterized by (different levels of) exposure to childhood adversity.

Third, future research will benefit from taking a biopsychosocial perspective, thus, acknowledging the complex interplay within and between different dimensions. Research taking a single-factor approach and/or based on examining isolated effects without addressing the environment and/or (obvious) confounding factors that could possibly interact, is thought to be explorative at best, and without use at worst. 
Fourth, while the impact of childhood adversity has been extensively researched, far less is known about protective factors and mechanisms of resilience that may prevent subsequent (health) problems or even promote 'post-traumatic growth'. Subsequently, identification of protective factors that buffer children from disruptions in emotional, social, cognitive, and neurobiological development following exposure to early life stress, is thought to be a critical next step for the field and, moreover, essential to guide the development of effective interventions to prevent the onset of psychopathology in children and adults with a history of childhood adversity. Similarly, further investigation of mechanisms of resilience in relation to adverse events is thought to be important since it can provide meaningful insights with respect to dealing with the aftermath of having been exposed to stressful and/or traumatic events.

Fifth, arguably one of the biggest limiting factors with respect to research in general and enhancing current knowledge regarding mental illness specifically, is what could be termed 'assumed knowledge' perpetuated by 'group thinking'. Within the field of psychiatry, over the last decades many researchers have built their studies around assumptions that have proven to be false, or at best only partially true. In fact, this still happens up until today. Some of these assumptions include: 'a specific diagnosis entails a specific cause', 'a specific diagnosis is clearly distinct from another diagnosis', 'mental disorders are caused by a small number of factors', and 'biological factors have primacy over environmental factors'.

Given the fact that such assumptions do not reflect reality, it is thought that research based on such assumptions is very limited in its capacity to provide meaningful answers. Therefore, it is assumed that research based on assumption-free designs, moreover, driven by bottom-up experiential knowledge concerning mental illness, is helpful in elucidating etiological mechanisms and providing valuable insights.

Last, although the work in this dissertation consistently demonstrated long-lasting impact of childhood adversity on several dimensions, we can only speculate about underlying mechanisms. Future research, preferably combining expertise from different scientific fields and people with lived experience, is thus necessary to advance our knowledge with respect to childhood adversity, the pathways involved causing subsequent dis-ease and dis-order, and finally, how to effectively address and treat its biopsychosocial legacy. 


\section{Clinical practice}

It goes without saying that childhood adversity can cause long-lasting detrimental effects on all aspects related to health and well-being. It concerns a topic that is extremely relevant to health care professionals specifically, and, arguably, all persons dealing with human beings in general.

First, as described earlier, childhood adversity may play a crucial role in the etiology of both physical and mental health problems, making it important to: 1) identify, and 2) adequately address the adversity and its consequences. With respect to the former, reducing the impact of childhood adversity starts with identifying it as early as possible, preferably during childhood itself. A major barrier to early detection, is that it relies primarily on voluntary disclosure of adversity by the individuals it concerns, which, as research suggests, is unlikely, particularly in the environment of health services. ${ }^{45-47}$ Thus, given that spontaneous disclosure is unlikely, it is the clinician's responsibility to enquire about past adversity. Despite this fact, routine enquiry about stressful and/or traumatic experiences in life is not common practice within health care settings. ${ }^{47,48}$

As a consequence, childhood adversity is thought to go undetected relatively often and, moreover, to go untreated too often. With respect to the latter, research suggests that it is important to not only train clinicians in how to address adversity, thereby increasing their confidence to ask and talk about stressful experiences, but moreover, that it is essential to bring about a fundamental shift towards trauma-informed health care and trauma-based understanding of patients' experiences, which, in turn, is thought to facilitate clinicians' commitment to routine enquiry of childhood adversity. ${ }^{48}$

This dissertation supports such a shift, given that it demonstrated long-lasting impact of childhood adversity in a general population at the level of everyday life, in relation to both mental and physical health and, moreover, by stressing the importance of addressing the context in which symptoms developed.

For clinical practice, this implies that it is of utmost importance to adopt routine enquiry of adverse events (both in mental and physical health care), as well as enhance the education of health care professionals and improve their general knowledge regarding childhood adversity. Knowledge of the impact of exposure to stressful and/or traumatic experiences is highly relevant to (mental) health providers, as is awareness of the diversity in subsequent stress- and trauma-related psychopathology. This is thought to be a necessary condition to be able to identify children enduring adverse childhoods. Ideally, such knowledge should be made available and receive attention within all professions that involve attending to children (e.g., schools and day-care centers) and 
people known to have certain disabilities and/or vulnerabilities (e.g., social services) specifically, and, arguably, people dealing with other people in general, since it is a matter that directly or indirectly concerns us all.

Second, in line with the above, I argue that health care professionals concerned with diagnosing and treating people, should always take into account the fact that childhood adversity may play a crucial role in both the causation as well as the maintenance of health problems later in life. This may be an obvious link, as is for instance the case between trauma and PTSD, but it can also be less obvious at first sight, especially when mediating pathways come into play that indirectly cause subsequent pathology.

Moreover, it should be recognized that what is sometimes considered to be a disorder, may in fact not be a problem, but a solution to a problem, or dis-order, and should thus be regarded as such. This can be exemplified by focusing on the link that has been observed between obesity and childhood sexual abuse, ${ }^{49-51}$ especially when considering the fact that, from a subjective perspective, being obese could be experienced as being unattractive and unnoticed, hence, being less vulnerable to becoming a victim of sexual abuse once again. Accordingly, recognition of the fact that symptoms (e.g., self-harming behavior or over-eating) can serve an important purpose (e.g., numb out feelings or self-regulate stress) is thought to be crucial, since it can have profound implications for addressing and treating mental illness and ill-health in general.

This notion is thought to be especially relevant with respect to public health campaigns that focus on 'unhealthy behaviors' such as overeating or physical inactivity. It is thus argued that campaigns aiming to deal with a problem without acknowledging that it may also reflect a solution, and, failing to address this fact, are ineffective and a waste of public funds. With respect to public health campaigns and interventions, I highly recommend that they take a trauma-informed approach, acknowledging the fact that there is (for instance) more to obesity than simply consuming more calories than one can burn off.

Third, this dissertation supports the notion that childhood adversity does not only affect the mind, but also the body. It furthermore supports the notion that dysregulation of the normal stress response, for instance manifesting as heightened sensitivity to stress and increased reactivity to stress, may form a central pathway in the trajectory from childhood adversity to later mental illness. An essential implication of this finding concerns the fact that it stresses the importance of implementing interventions and treatment methods that address the body and the impact left on the body or, stated differently, that take a body-oriented approach. 
While, as argued above, cognitive-behavioral therapy (i.e., the golden standard of psychotherapy) may be beneficial with respect to treating certain (cognitive) symptoms, it is considered insufficient to deal with the sequelae of childhood adversity, that, arguably, to a great extent are stored within and experienced through the body.

Accordingly, this dissertation supports the notion that mental health care, and its users specifically, would benefit greatly from implementing more body-oriented treatment methods, such as for instance sensorimotor psychotherapy, ${ }^{52}$ body-oriented or somaticoriented psychotherapy, ${ }^{53}$ psychomotor therapy, ${ }^{54}$ and somatic experiencing. ${ }^{55}$ In addition to including more body-oriented approaches, it is furthermore recommended to also include more expressive and non-verbal approaches, such as dance and

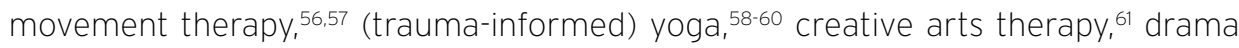
therapy, ${ }^{62}$ and animal assisted therapy. ${ }^{63}$

One of the main arguments for implementing more body-oriented and non-verbal treatment methods, concerns the fact that extremely stressful experiences can affect the brain in such a way, that it becomes virtually impossible to verbally communicate an experience sometimes referred to as 'speechless horror'. ${ }^{58}$ This may happen during the stressful experience itself, as well as (long) after, when (implicit) memories of the event cause the speech center in the brain (i.e., Broca's area) to go 'offline'. Without a functioning Broca's area, then, one simply cannot put thoughts and feelings into words. Hence, for the treatment of traumatized people, it is considered important to apply more expressive and non-verbal treatment methods. This enables ways of communication that do not require speech or narratives, but can be extremely valuable, nevertheless.

Fourth, in accordance with the recommendation above to use more body-oriented and non-verbal approaches, I argue that in treatment, one of the most important aims should be to learn people how to tolerate feelings and distress without moving to hide it, fade it, or fix it. More specifically, teaching people skills to self-regulate their emotions and nervous system so they can move from dis-ease to ease, is thought to be a vital component in treating people who suffer the consequences of having endured adversity early in life. Often, these skills are significantly impaired or even entirely lacking, as they might have never been developed in the first place. It is thought that learning such skills require body-oriented and non-verbal methods, since it is arguably more a matter of the body (and getting to know one's body), than it is of the mind. 
Accordingly, it is furthermore argued that schools can play a fundamental role in teaching children self-regulating skills from an early age, thereby instilling resilience that can buffer against adverse consequences from experiences later on. At school, children can easily be taught how their brains and bodies work, and how they can understand and deal with their emotions. As a consequence, this may protect them from future damage done by exposure to (extremely) stressful circumstances, since they may be better equipped to deal with the impact.

To conclude, in addition to reading, writing and counting, it is highly recommended that all children learn about self-awareness, self-regulation and communication skills, from the earliest age possible. Thus, I argue that it should be a core element in every school curriculum. In the end, it is easier to build strong children, than to repair broken adults. 


\section{FINAL THOUGHTS}

This dissertation has investigated underlying mechanisms that may contribute to the emergence of mental illness, specifically focusing on the trajectory from childhood adversity to mental ill-health later in life. Results supports the notion that childhood adversity does not directly impact on health and well-being, but involves an indirect, mediating and/or moderating effect. Hence, the impact of childhood adversity on mental health is thought to operate through various different biopsychosocial pathways, possibly involving a central pathway of heightened sensitivity to stress and increased stress reactivity. Additionally, the body - and all the biological processes it embodies - is thought to play a fundamental role. Consequently, with respect to the treatment of (mental) health problems following childhood adversity, application of more bodyoriented and non-verbal treatment approaches is recommended, specifically aimed at teaching people how to self-regulate their emotions and nervous system.

Taking all of the above into account, perhaps the most safe and solid conclusion that can be drawn, is that mental illness involves boundless complexity. This is evidenced by the fact that, in the first place, mental illness can manifest in countless different shapes and forms. In the second place, there are countless biopsychosocial factors and equally countless possible interactions between these factors that can contribute to the onset of ill-health. Hence, with respect to addressing public health and providing the general population with valuable knowledge about mental health and mental disorders, it is of importance to practice modesty and stay away from statements and conclusions for which there is no, or little, convincing evidence. Arguably, acknowledging what we do not know might be even more important than what we do know. Or, as the statement attributed to Confucius says:

"To know what you know, and what you do not know, that is true knowledge."64 


\section{REFERENCES}

1. Uher R, Zwicker A. Etiology in psychiatry: embracing the reality of poly-gene-environmental causation of mental illness. World Psychiatry Jun 2017;16(2):121-129.

2. Guinosso SA, Johnson SB, Riley AW. Multiple adverse experiences and child cognitive development. Pediatr Res Jan 2016;79(1-2):220-226.

3. Yang L, Wang Z. Early-Life Conditions and Cognitive Function in Middle-and Old-Aged Chinese Adults: A Longitudinal Study. Int J Environ Res Public Health May 15 2020;17(10).

4. Aas M, Dazzan P, Mondelli V, Melle I, Murray RM, Pariante CM. A systematic review of cognitive function in first-episode psychosis, including a discussion on childhood trauma, stress, and inflammation. Front Psychiatry Jan 8 2014;4:182.

5. Sideli L, Fisher HL, Russo M, et al. Failure to find association between childhood abuse and cognition in first-episode psychosis patients. Eur Psychiatry Jan 2014;29(1):32-35.

6. Fervaha G, Zakzanis KK, Foussias G, Graff-Guerrero A, Agid O, Remington G. Motivational deficits and cognitive test performance in schizophrenia. JAMA Psychiatry Sep 2014;71(9):1058-1065.

7. Lardinois M, Lataster T, Mengelers R, Van Os J, Myin-Germeys I. Childhood trauma and increased stress sensitivity in psychosis. Acta Psychiatr Scand Jan 2011;123(1):28-35.

8. Wichers M, Schrijvers D, Geschwind N, et al. Mechanisms of gene-environment interactions in depression: evidence that genes potentiate multiple sources of adversity. Psychol Med Jul 2009;39(7):1077-1086.

9. Reich CG, Taylor ME, McCarthy MM. Differential effects of chronic unpredictable stress on hippocampal CB1 receptors in male and female rats. Behav Brain Res Nov 5 2009;203(2):264-269.

10. Selye $H$. The nature of stress. Basal Facts 1985;7(1):3-11.

11. Luijcks R, Hermens HJ, Bodar L, Vossen CJ, Van Os J, Lousberg R. Experimentally induced stress validated by EMG activity. PLoS One 2014;9(4):e95215.

12. Luijcks R, Vossen CJ, Roggeveen S, van Os J, Hermens HJ, Lousberg R. Impact of early life adversity on EMG stress reactivity of the trapezius muscle. Medicine (Baltimore) Sep 2016;95(39):e4745.

13. Rice ASC, Smith BH, Blyth FM. Pain and the global burden of disease. Pain Apr 2016:157(4):791-796.

14. Fayaz A, Croft P, Langford RM, Donaldson LJ, Jones GT. Prevalence of chronic pain in the UK: a systematic review and meta-analysis of population studies. BMJ Open Jun 20 2016;6(6):e010364.

15. Scott KM, Von Korff M, Angermeyer MC, et al. Association of childhood adversities and earlyonset mental disorders with adult-onset chronic physical conditions. Archives of general psychiatry 2011;68(8):838-844.

16. Kessler RC, McLaughlin KA, Green JG, et al. Childhood adversities and adult psychopathology in the WHO World Mental Health Surveys. The British journal of psychiatry : the journal of mental science 2010;197(5):378-385.

17. Noteboom A, Have MT, de Graaf R, Beekman ATF, Penninx B, Lamers F. The long-lasting impact of childhood trauma on adult chronic physical disorders. J Psychiatr Res Apr 2021;136:87-94.

18. McLaughlin KA. Future Directions in Childhood Adversity and Youth Psychopathology. J Clin Child Adolesc Psychol 2016;45(3):361-382.

19. Krupnik V. Trauma or adversity? Traumatology 2019;25(4):256-261.

20. American Psychiatric Association. Diagnostic and Statistical Manual of Mental Disorders. 5th ed. Washington, DC; 2013.

21. Teicher MH, Samson JA, Polcari A, McGreenery CE. Sticks, stones, and hurtful words: relative effects of various forms of childhood maltreatment. Am J Psychiatry Jun 2006;163(6):993-1000. 
22. Daniel S. Schechter, M.D. The Developmental Neuroscience of Emotional Neglect, Its Consequences, and the Psychosocial Interventions That Can Reverse Them. American Journal of Psychiatry 2012;169(5):452-454.

23. Seery MD, Holman EA, Silver RC. Whatever does not kill us: cumulative lifetime adversity, vulnerability, and resilience. Journal of personality and social psychology 2010;99(6):1025-1041.

24. Krupnik V. Trauma or Drama: A Predictive Processing Perspective on the Continuum of Stress. Frontiers in psychology 2020;11:1248-1248.

25. Dong M, Anda RF, Felitti VJ, Dube SR, Williamson DF, Thompson TJ, Loo CM, Giles WH. The interrelatedness of multiple forms of childhood abuse, neglect, and household dysfunction. Child Abuse Negl Jul 2004;28(7):771-784.

26. Evans GW, Li D, Whipple SS. Cumulative risk and child development. Psychol Bull Nov 2013;139(6):13421396.

27. Yaribeygi H, Panahi Y, Sahraei H, Johnston TP, Sahebkar A. The impact of stress on body function: A review. EXCLI J 2017:16:1057-1072.

28. van Nierop M, Lecei A, Myin-Germeys I, et al. Stress reactivity links childhood trauma exposure to an admixture of depressive, anxiety, and psychosis symptoms. Psychiatry Res Feb 2018;260:451457.

29. Evans GW, De France K. Childhood poverty and psychological well-being: The mediating role of cumulative risk exposure. Dev Psychopathol Feb 2 2021:1-11.

30. David D, Cristea I, Hofmann SG. Why Cognitive Behavioral Therapy Is the Current Gold Standard of Psychotherapy. Frontiers in psychiatry 2018:9:4-4.

31. Hofmann SG, Asmundson GJ, Beck AT. The science of cognitive therapy. Behav Ther Jun 2013:44(2):199-212.

32. Ohrnberger J, Fichera E, Sutton M. The relationship between physical and mental health: A mediation analysis. Social Science \& Medicine 2017/12/01/ 2017;195:42-49.

33. de Witte $L$, de Jel $D$, van Mierlo $H$, Witte M, Vinkers C. Herkenning van somatische oorzaken van psychose en stemmingsstoornissen: een systematisch overzicht. 09/07 2018.

34. Damasio AR. Emotions and Feelings: A Neurobiological Perspective. Feelings and emotions: The Amsterdam symposium. New York, NY, US: Cambridge University Press; 2004:49-57.

35. Rauschenberg C, van Os J, Cremers D, Goedhart M, Schieveld JNM, Reininghaus U. Stress sensitivity as a putative mechanism linking childhood trauma and psychopathology in youth's daily life. Acta Psychiatr Scand Oct 2017:136(4):373-388.

36. Shonkoff JP, Boyce WT, McEwen BS. Neuroscience, Molecular Biology, and the Childhood Roots of Health Disparities: Building a New Framework for Health Promotion and Disease Prevention. JAMA 2009:301(21):2252-2259.

37. Felitti VJ, Anda RF, Nordenberg D, Williamson DF, Spitz AM, Edwards V, Koss MP, Marks JS Relationship of childhood abuse and household dysfunction to many of the leading causes of death in adults. The Adverse Childhood Experiences (ACE) Study. Am J Prev Med May 1998;14(4):245-258.

38. Ford E, Clark C, Stansfeld SA. The influence of childhood adversity on social relations and mental health at mid-life. Journal of Affective Disorders 2011/09/01/2011;133(1):320-327.

39. Santini ZI, Koyanagi A, Stewart-Brown S, Perry BD, Marmot M, Koushede V. Cumulative risk of compromised physical, mental and social health in adulthood due to family conflict and financial strain during childhood: a retrospective analysis based on survey data representative of 19 European countries. BMJ Global Health 2021;6(3):e004144.

40. Shah AK, Mullainathan S, Shafir E. Some Consequences of Having Too Little. Science 2012;338(6107):682-685. 
41. Scheffers M, Hoek M, Bosscher RJ, van Duijn MAJ, Schoevers RA, van Busschbach JT. Negative body experience in women with early childhood trauma: associations with trauma severity and dissociation. Eur J Psychotraumatol 2017:8(1):1322892.

42. Ogden P, Minton K, Pain C. Trauma and the body : a sensorimotor approach to psychotherapy. 1st ed. New York: W.W. Norton; 2006.

43. Fazio RH, OIson MA. Attitudes: Foundations, functions, and consequences. In: Hogg MA, Cooper J, eds. The Sage handbook of social psychology. Londen: Sage; 2003:139-160.

44. Mehling WE, Gopisetty V, Daubenmier J, Price CJ, Hecht FM, Stewart A. Body awareness: construct and self-report measures. PLoS One 2009;4(5):e5614.

45. John Read, Ph.D., and, Allen Fraser, M.R.C.Psych., F.R.A.N.Z.P. Abuse Histories of Psychiatric Inpatients: To Ask or Not to Ask? Psychiatric Services 1998;49(3):355-359.

46. Lothian J, Read J. Asking about Abuse during Mental Health Assessments: Clients' Views and Experiences. New Zealand Journal of Psychology 2002;31(2):98-103.

47. Hepworth I, McGowan L. Do mental health professionals enquire about childhood sexual abuse during routine mental health assessment in acute mental health settings? A substantive literature review. Journal of Psychiatric and Mental Health Nursing 2013;20(6):473-483.

48. Pearce J, Murray C, Larkin W. Childhood adversity and trauma: experiences of professionals trained to routinely enquire about childhood adversity. Heliyon 07/01 2019;5:e1900.

49. Felitti V, Anda R. The Relationship of Adverse Childhood Experiences to Adult Medical Disease, Psychiatric Disorders, and Sexual Behavior: Implications for Healthcare. The Impact of Early Life Trauma on Health and Disease: The Hidden Epidemic 01/01 2009.

50. Gustafson TB, Sarwer DB. Childhood sexual abuse and obesity. Obesity Reviews 2004;5(3):129-135.

51. Evans GW, Fuller-Rowell TE, Doan SN. Childhood cumulative risk and obesity: the mediating role of self-regulatory ability. Pediatrics Jan 2012;129(1):e68-73.

52. Fisher J. Sensorimotor psychotherapy in the treatment of trauma. Practice Innovations 2019:4(3):156-165.

53. Röhricht F. Body oriented psychotherapy. The state of the art in empirical research and evidencebased practice: A clinical perspective. Body, Movement and Dance in Psychotherapy 08/01 2009:4:135-156.

54. Emck C, Scheffers M. Psychomotor interventions for mental health: An introduction. Psychomotor interventions for mental health: Boom; 2019:17-51.

55. Levine PA. In an unspoken voice: How the body releases trauma and restores goodness: North Atlantic Books; 2010.

56. Stromsted T, Sieff D. Dances of Psyche and Soma: Re-inhabiting the Body in the wake of Emotional Trauma. 2015:46-63.

57. Grasser LR, Al-Saghir H, Wanna C, Spinei J, Javanbakht A. Moving Through the Trauma: Dance/ Movement Therapy as a Somatic-Based Intervention for Addressing Trauma and Stress Among Syrian Refugee Children. J Am Acad Child Adolesc Psychiatry Nov 2019;58(11):1124-1126.

58. Van der Kolk BA. The body keeps the score : brain, mind, and body in the healing of trauma. New York: Viking; 2014.

59. Justice L, Brems C, Ehlers K. Bridging Body and Mind: Considerations for Trauma-Informed Yoga. International Journal of Yoga Therapy 2018;28(1):39-50.

60. Emerson D, Hopper E. Overcoming trauma through yoga: Reclaiming your body: North Atlantic Books; 2012.

61. Perryman K, Blisard P, Moss R. Using Creative Arts in Trauma Therapy: The Neuroscience of Healing. Journal of Mental Health Counseling 2019;41(1):80-94. 
62. Sajnani N, Johnson DR. Trauma-informed drama therapy: Transforming clinics, classrooms, and communities: Charles C Thomas Publisher; 2014.

63. Mims D, Waddell R. Animal Assisted Therapy and Trauma Survivors. Journal of Evidence-Informed Social Work 2016/09/02 2016;13(5):452-457.

64. Sosa E. Confucius on Knowledge. Dao : A Journal of Comparative Philosophy 2015;14(3):325-330. 




\title{
ADDENDUM
}

\author{
SUMMARY \\ SAMENVATTING \\ IMPACT PARAGRAPH \\ CURRICULUM VITAE \\ LIST OF PUBLICATIONS \\ DANKWOORD
}




\section{SUMMARY}

The central aim of this dissertation was to investigate underlying mechanisms that may contribute to the emergence of mental illness, specifically focusing on the trajectory from childhood adversity to mental ill-health later in life. For this purpose, the contribution of both genetic and environmental factors in relation to mental health was explored, emphasizing the role of childhood adversity by examining its long-lasting impact at the cognitive, psychological, and psychophysiological level, as well as at the epidemiological level of transition from health to ill-health.

Chapter 1 introduced the main topic by first briefly addressing the Dutch 'Schizophrenia does not exist' campaign of which the author formed part of, followed by a short review of the perspective on (ill) health through a biomedical model and biopsychosocial model, and finally focusing on childhood adversity and its long-lasting impact on (mental) health. The introduction finished with an outline of the different studies in this dissertation and their objectives.

Chapter $\mathbf{2}$ explored the contribution of molecular genetic, familial, and environmental risk factors to the variance in level of and change in mental health in a large populationbased cohort. The cohort was examined four times over a period of nine years. In a regression model, polygenic risk scores (PRS) and several measures of environmental risks (including childhood adversity) and social circumstances were included to calculate the relative contribution of each (group of) risk factor(s) at the level of and change in general mental health. Around $17 \%$ of the variance in mental health could be explained by familial and environmental factors, while only $0.4 \%$ could be explained by PRS for schizophrenia (PRS-SZ). Childhood adversity, representing an environmental factor, had the largest impact. Results were similar, but attenuated, for the model of mental health change over time.

Chapter 3 focused on the link between childhood adversity and cognition, investigating the relationship in a longitudinal study that included people with psychotic disorder, their siblings and healthy comparison subjects. The study sample was interviewed three times over a period of six years, allowing for the analyses of repeated measures of $I Q$ as a function of childhood adversity and group. Significant differences were found in the impact of childhood adversity on $I Q$ across the three groups. Exposure to childhood adversity was associated with a nearly 5-point reduction in IQ in healthy controls, a lesser reduction (2.58) in siblings, and no significant reduction in patients. With respect to the relationship between childhood adversity and learning abilities, childhood adversity was found 
to negatively impact on the course of $I Q$ over time for all three groups. Those with exposure to childhood adversity showed significantly less learning effects with repeated cognitive assessments than the non-exposed.

Chapter $\mathbf{4}$ and chapter $\mathbf{5}$ explored the relationship between childhood adversity and (increased) stress sensitivity by conducting two different stress experiments. In both experiments, the first a memory task using a cognitive stressor (chapter $\mathbf{4}$ ), the second a habituation task using a series of electrical painful stimuli (chapter 5), it was investigated how adverse childhood experiences (ACE) may impact electromyography (EMG) activity of the trapezius muscle. To investigate the role of predictability in the experiment, both study protocols included an identical session six months after the first session. For both tasks, a significant session effect was found (i.e., decreased EMG reactivity during the second session compared to the first), likely due to a reduction in unpredictability during the second session. Additionally, with respect to the influence of childhood adversity, a moderating effect of ACE on stress-induced EMG activity was observed: higher ACE scores resulted in greater EMG reactivity. Finally, a dose-response relationship was found, demonstrating that participants with higher ACE scores showed increased EMG reactivity relative to those with lower ACE scores.

In addition to examining the association between ACE and stress-related muscle reactivity, chapter $\mathbf{5}$ moreover examined the impact of ACE on habituation to pain. The impact of ACE was assessed at an objective level (EMG) as well as a subjective level (pain report on a numeric rating scale). With respect to the objective level, results indicated that higher ACE-scores were associated with diminished habituation, both in the short term (i.e., at the inter-stimulus-interval level) as well as in the longer term (i.e., at the stimulus number and session level) and, thus, occasioning a within-session and a between-session habituation effect. Further, NRS scores were also significantly moderated by childhood adversity, such that participants with a history of ACE, on average, reported higher NRS scores relative to those without a history of ACE. In addition, over the course of 25 stimuli, the scores of people with ACE did not decrease as much as for the non-ACE group.

Chapter $\mathbf{6}$ summarized and discussed the main findings of this dissertation, followed by several in-depth considerations and two proposed models to illustrate how childhood adversity may affect the relationship between mental and physical health in general, and the association between childhood adversity and mental health more specifically. Finally, based on both the work presented in this dissertation, as well as on the lived experience of the author, a number of recommendations were offered for future research and clinical practice. 
In conclusion, this dissertation supports the notion that childhood adversity does not directly impact on health and well-being, but involves an indirect, mediating and/ or moderating effect. Hence, the impact of childhood adversity on mental health is thought to operate through various different biopsychosocial pathways, possibly involving a central pathway of heightened sensitivity to stress and increased stress reactivity. Additionally, the body - and all the biological processes it embodies - is thought to play a fundamental role that requires further understanding, addressing, and acknowledging. 


\section{SAMENVATTING}

\section{Beyond dis-ease and dis-order: een exploratie van de lange termijn gevolgen van ingrijpende gebeurtenissen in de jeugd in relatie tot mentale gezondheid}

In dit proefschrift zijn onderliggende mechanismen onderzocht die mogelijk een rol spelen in het ontstaan van psychische problemen, met een specifieke focus op de relatie tussen ingrijpende gebeurtenissen in de jeugd (adverse childhood experiences, ACE) en psychische problemen in het latere leven. Van zowel genetische- als omgevingsfactoren is onderzocht welke bijdrage zij leveren aan mentale gezondheid. De nadruk werd gelegd op de rol van ACE door de lange termijn impact ervan te onderzoeken op cognitief, psychologisch en psychofysiologisch niveau, als ook op epidemiologisch niveau, door te kijken naar de overgang van gezondheid naar ziekte.

Hoofdstuk 1 introduceerde het centrale thema door eerst kort in te gaan op de 'Schizofrenie bestaat niet' campagne waar de auteur deel van uitmaakte, gevolgd door een korte beschrijving van het perspectief op gezondheid en ziekte vanuit een biomedisch en biopsychosociaal model, en richtte zich tenslotte op ingrijpende gebeurtenissen in de jeugd en de langdurige gevolgen ervan op de (mentale) gezondheid. De inleiding werd afgesloten met een overzicht van de verschillende studies die in dit proefschrift zijn opgenomen en hun doelstellingen.

Hoofdstuk 2 onderzocht de impact van genetische, familiale en omgevingsrisicofactoren op zowel het niveau van, als de verandering in, mentale gezondheid. Het onderzoek werd verricht onder een grote groep van de algemene Nederlandse bevolking, die gedurende negen jaar vier keer werd geïnterviewd. In een regressiemodel werden polygene risicoscores (PRS) en verschillende maten van omgevingsrisico's (inclusief ACE) en sociale omstandigheden opgenomen om de relatieve bijdrage van elke (groep van) risicofactor(en) op het niveau van, en verandering in, algemene mentale gezondheid te berekenen. Ongeveer 17\% van de variantie in mentale gezondheid kon worden verklaard door familiale en omgevingsfactoren, terwijl slechts 0,4\% kon worden verklaard door PRS voor schizofrenie (PRS-SZ). Ingrijpende gebeurtenissen in de jeugd, een omgevingsfactor, had de grootste impact. Voor het model van veranderingen in de mentale gezondheid in de loop van de tijd waren de resultaten vergelijkbaar, maar zwakker.

Hoofdstuk 3 richtte zich op het verband tussen ingrijpende gebeurtenissen in de jeugd en cognitie. Dit verband werd onderzocht in een longitudinaal onderzoek onder mensen met een psychotische stoornis, hun broers en zussen en gezonde controles. De onderzoekspopulatie werd gedurende zes jaar drie keer geïnterviewd. Er werden 
significante verschillen gevonden qua impact van ACE op IQ tussen de drie groepen. Blootstelling aan ACE was geassocieerd met een afname van bijna 5 punten in IQ bij gezonde controles, een kleinere afname $(2,58)$ bij broers en zussen en geen significante afname bij mensen met een psychotische stoornis. Met betrekking tot de relatie tussen ACE en leervermogen, bleek voor alle drie de groepen een negatieve invloed op het beloop van het IQ over de tijd. Degenen met blootstelling aan ACE vertoonden significant minder leereffecten bij herhaalde cognitieve beoordelingen dan de nietblootgestelde personen.

Hoofdstuk 4 en hoofdstuk 5 onderzochten de relatie tussen ACE en stressgevoeligheid door twee verschillende stressexperimenten uit te voeren. In beide experimenten, de eerste een geheugentaak waarin een cognitieve stressor werd gebruikt (hoofdstuk 4), de tweede een gewenningstaak waarin een reeks elektrische pijnprikkels werden aangeboden (hoofdstuk 5), werd onderzocht hoe ACE van invloed kan zijn op elektromyografie (EMG) activiteit van de trapeziusspier. Om de rol van voorspelbaarheid in het experiment te onderzoeken, presenteerden beide studies de resultaten van een identieke sessie zes maanden na de eerste sessie. Voor beide taken werd een significant sessie-effect gevonden (d.w.z. verminderde EMG-reactiviteit tijdens de tweede sessie in vergelijking met de eerste), waarschijnlijk als gevolg van toegenomen voorspelbaarheid tijdens de tweede sessie. Bovendien werd een modererend effect van ACE op stressgeïnduceerde EMG-activiteit waargenomen: hogere ACE-scores resulteerden in grotere EMG-reactiviteit. Ten slotte werd een dosis-responsrelatie gevonden: deelnemers met hogere ACE-scores vertoonden verhoogde EMG-reactiviteit in vergelijking met degenen met lagere ACE-scores.

Naast het onderzoeken van de associatie tussen ACE en stress-gerelateerde spierreactiviteit, onderzocht hoofdstuk 5 bovendien de impact van ACE op gewenning aan pijn. De impact van ACE werd zowel op objectief niveau (EMG) als op subjectief niveau (pijnscore op een numerieke beoordelingsschaal) onderzocht. Op EMG-niveau lieten de resultaten zien dat hogere ACE-scores geassocieerd zijn met verminderde gewenning, zowel op de korte termijn (d.w.z. op het inter-stimulus-intervalniveau) als op de langere termijn (d.w.z. op het stimulusnummer-en sessieniveau). Op het subjectieve niveau van pijnscores werd eveneens een modererend effect van ACE aangetoond: mensen met een voorgeschiedenis van ingrijpende ervaringen rapporteerden gemiddeld hogere pijnscores in vergelijking met degenen zonder ACE. Daarnaast daalden de pijnscores gedurende het experiment bij mensen met ACE minder dan voor de niet-ACE-groep. 
Hoofdstuk 6 beschreef de belangrijkste bevindingen van dit proefschrift, gevolgd door een aantal diepgaande overwegingen en twee voorgestelde modellen om te illustreren hoe ingrijpende gebeurtenissen in de jeugd de relatie tussen mentale en fysieke gezondheid in het algemeen kunnen beïnvloeden, en meer specifiek de relatie tussen ingrijpende gebeurtenissen en mentale gezondheid. Ten slotte werden, op basis van zowel de studies die in dit proefschrift zijn gepresenteerd als de eigen ervaring van de auteur, een aantal aanbevelingen gedaan voor toekomstig onderzoek en de klinische praktijk.

Concluderend ondersteunt dit proefschrift het idee dat ingrijpende gebeurtenissen in de jeugd niet een direct effect hebben op gezondheid en welzijn, maar eerder een indirect, mediërend en/of modererend effect. Verondersteld wordt dat de impact van ingrijpende gebeurtenissen op de mentale gezondheid via verschillende biopsychosociale paden verloopt, waarbij verhoogde gevoeligheid voor stress en verhoogde stressreactiviteit mogelijk centrale mechanismen vormen. Tevens wordt verondersteld dat het lichaam - en alle biologische processen die het belichaamt - een fundamentele rol speelt die verdere adressering, verkenning en erkenning vereist. 


\section{IMPACT PARAGRAPH}

Perhaps the best way to illustrate the relevance and impact of the work presented in this dissertation, is by connecting its findings to the global situation we find ourselves currently faced with.

In January 2020, the World Health Organization (WHO) declared the new coronavirus disease, termed COVID-19, to be a 'Public health emergency of international concern'. Consequently, severe measures were taken all around the globe in an attempt to stop the virus from spreading. Entire nations were forced to stay home, close their businesses, and keep distance from one another, which, inevitably had enormous impact on the daily lives of people. Given all these measures, as well as the elevated levels of fear, worry and concern directly or indirectly caused by the pandemic, it was only a matter of time for the 'second pandemic' to arise. Namely, the pandemic of impaired mental health. ${ }^{2}$ Being aware of the psychosocial impact of COVID-19, the WHO started to communicate about mental health concerns already shortly after declaring the health emergency in January, expecting levels of loneliness, depression, harmful alcohol and drug use, and self-harm or suicidal behaviors to rise. ${ }^{3}$ They furthermore wrote on their website: "Fear, worry, and stress are normal responses to perceived or real threats, and at times when we are faced with uncertainty or the unknown. So it is normal and understandable that people are experiencing fear in the context of the COVID-19 pandemic."4

Indeed, it can be considered normal and understandable that people experience fear and stress in the context of a pandemic, which, arguably, is best considered a huge biopsychosocial stressor that, additionally, involves a high degree of uncertainty and uncontrollability - aspects known to hold the potential to significantly impact on health and well-being. More specifically, on the health and well-being of individuals who are already vulnerable to the impact of stress and thus most likely to suffer adverse consequences. Based on the work presented in this dissertation, I argue that it may very well be that people who have been exposed to traumatic or toxic stress (early) in their lives, are the ones most threatened by the stressful circumstances we are faced with today. They are threatened both directly and indirectly, thus vulnerable to the stressors related to the COVID-19 pandemic, as well as to other daily life stressors that may be considered minor or irrelevant to many, yet can nevertheless be very distressing to some. 
With respect to the above, perhaps one of the most important implications of this dissertation, is that it stresses the notion that the so called 'vulnerable groups' often referred to in the media, are not limited to those of old age, or those in poor physical health, but moreover include people with a history of childhood adversity, and adversity in general. Thus, I argue that in societies where people are continuously and sometimes chronically exposed to some level of stress, as is for instance the case in the current COVID-19 pandemic, it is of utmost importance to expand our knowledge and understanding of the consequences of stress on health and well-being, as well as identifying the people who are most vulnerable to bear these consequences. This is necessary to prevent subsequent suffering, and to be able to respond effectively once damage is done to the brain, mind, and body.

By exploring the long-lasting impact of childhood adversity in a general population, and, moreover, by shining light on the complex interplay of biological, psychological, and social dimensions that are known to influence all aspects of human life, this dissertation aimed to do exactly that: expand our knowledge and understanding of the long-lasting consequences of (early life) stress on health in general, and mental health more specifically. The findings and subsequent recommendations presented here are thus considered to be highly relevant on a societal level, but perhaps even more so on the individual level of people who have endured childhood adversity themselves, and who are still suffering from the consequences up until today.

In conclusion, it is my deepest wish that the work in this dissertation encourages people to always look behind the surface and to start seeing beyond dis-order and dis-ease by investigating what their past may have to do with their present, and what steps there are to take to build a brighter future. If it accomplishes this, even if for one individual, I consider my work impactful. 


\section{REFERENCES}

1. World Health Organization. 2019-nCoV outbreak is an emergency of international concern. 31 January 2020. Available at: https://www.euro.who.int/en/health-topics/health-emergencies/ coronavirus-covid-19/news/news/2020/01/2019-ncov-outbreak-is-an-emergency-of-internationalconcern. Accessed 28 April, 2021.

2. Choi KR, Heilemann MV, Fauer A, Mead M. A Second Pandemic: Mental Health Spillover From the Novel Coronavirus (COVID-19). Journal of the American Psychiatric Nurses Association 2020;26(4):340-343.

3. World Health Organization. Mental health and COVID-19. Available at: https://www.euro.who.int/ en/health-topics/health-emergencies/coronavirus-covid-19/publications-and-technical-guidance/ noncommunicable-diseases/mental-health-and-covid-19. Accessed 28 April, 2020.

4. World Health Organization. Mental health \& COVID-19. Available at: https://www.who.int/teams/ mental-health-and-substance-use/covid-19. Accessed 28 April 2021 


\section{CURRICULUM VITAE}

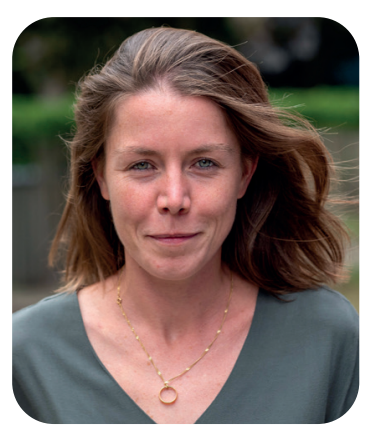

Anne Marsman werd geboren op 26 december 1989 en groeide op in Hoofddorp, waar ze het basisonderwijs volgde op een Vrije School. In 2008 behaalde ze haar diploma aan het Atheneum College Hageveld te Heemstede. Met haar diploma ontving Anne gevoelsmatig haar vrijheid en vertrok niet lang daarna via New York, waar ze op haar 18e de marathon liep, naar India. Daar volgde ze een aantal weken verschillende retraites in een boeddhistisch centrum aan de voet van de Himalaya, om vervolgens nog eens 1,5 jaar in Dechen Chöling, een boeddhistisch centrum in Frankrijk, te wonen, werken en zijn. Een plek waar ze zich thuis voelde, mensen van over de hele wereld ontmoette, en vooral ontzettend veel leerde over leven, liefde en lijden.

Na twee tussenjaren verhuisde ze in 2010 naar Maastricht om gezondheidswetenschappen te studeren. Daarnaast volgde ze het Honours programma 'International Health', waarvoor ze 6 weken naar India ging om over het gezondheidzorgsysteem aldaar te leren. Tijdens haar studie werkte Anne als buddy van een oudere dame met alzheimer en als balie- en redactiemedewerker van het universitaire sportcentrum. Door een samenloop van ongelukkige omstandigheden moest ze haar studie drie maanden voor de finish onderbreken, om een jaar later alsnog haar bachelor te halen. Ze kwam in contact met prof. dr. Jim van Os en ging niet lang daarna aan de slag als assistent psycholoog/onderzoeker. Zodoende implementeerde ze een op mHealth gebasseerde ROM (routine outcome monitoring) methode binnen de polikliniek psychiatrie van het MUMC, nam ze interviews af rondom psychotische ervaringen voor het NEMESISonderzoek en assisteerde ze bij verschillende lopende studies.

In het najaar van 2014 raakte Anne, min of meer bij toeval, betrokken bij de 'Schizofrenie Bestaat Niet' campagne. Ze hielp mee aan het realiseren van de website die op 7 maart 2015 het licht zag en werkte vervolgens jarenlang met hart en ziel als hoofdredacteur aan het daaruit voortkomende platform PsychoseNet.nl. Een platform dat in een aantal jaar tijd uitgroeide tot een van de meest succesvolle online hulpplatforms in Nederland, inmiddels goed voor ruim 1,5 miljoen unieke bezoekers per jaar.

Terwijl Anne zich meer dan fulltime inzette voor PsychoseNet, startte zij in 2016 ook met haar promotietraject bij de vakgroep Psychiatrie \& Neuropsychologie van de Universiteit Maastricht. Gezien haar eerdere betrokkenheid bij verschillende lopende 
studies en aan haar beschikbaar gestelde data, kon en mocht ze dit traject zowel qua inhoud als planning grotendeels zelf vormgeven. De eerste drie jaar van haar onderzoek vonden dan vooral ook 'tussen de bedrijven door' plaats. In 2017 haalde ze, eveneens 'tussen de bedrijven door' haar master klinische psychologie, waarna ze als psycholoog onder andere voor 113 Zelfmoordpreventie en binnen de ouderenpsychiatrie werkte.

Begin 2021 nam Anne na ruim zes jaar afscheid van PsychoseNet, om ruimte te maken voor nieuwe dromen, en een nieuw begin. Een nieuw begin dat begon met het afronden van haar proefschrift 'Beyond dis-ease and dis-order', waarin ze haar eigen ervaring met ingrijpende gebeurtenissen en psychische problematiek gecombineerd heeft met haar professionele en wetenschappelijke kennis rondom het thema trauma.

To be continued... 


\section{LIST OF PUBLICATIONS}

van Os J*, Marsman A*, van Dam D, Simons CJ; GROUP Investigators. Evidence That the Impact of Childhood Trauma on IQ Is Substantial in Controls, Moderate in Siblings, and Absent in Patients With Psychotic Disorder. Schizophr Bull. 2017 Mar 1;43(2):316-324.

Marsman A, Luijcks R, Vossen C, van Os J, Lousberg R. The impact of adverse childhood experiences on EMG reactivity: A proof of concept study. PLoS One. 2019; 14(5).

Marsman A, Pries LK, Ten Have M, de Graaf R, van Dorsselaer S, Bak M, Kenis G, Lin BD, Luykx JJ, Rutten BPF, Guloksuz S, van Os J. Do Current Measures of Polygenic Risk for Mental Disorders Contribute to Population Variance in Mental Health? Schizophr Bull. 2020; 46(6):1353-1362.

Marsman A, Luijcks R, Vossen C, van Os J, Lousberg R. The influence of childhood adversity on stress-sensitivity and habituation to pain. 2021.

*Shared authorship

\section{Not in this dissertation}

Verhagen SJW, Berben JA, Leue C, Marsman A, Delespaul PAEG, van Os J, et al. (2017) Demonstrating the reliability of transdiagnostic mHealth Routine Outcome Monitoring in mental health services using experience sampling technology. PLoS ONE 12 (10): e0186294.

van Os, J., Verhagen, S., Marsman, A., Peeters, F., Bak, M., Marcelis, M., Delespaul, P. (2017). The experience sampling method as an mHealth tool to support self-monitoring, self-insight, and personalized health care in clinical practice. Depression and Anxiety, 34(6), 481-493.

Broen MPG, Marsman VAM, Kuijf ML, Van Oostenbrugge RJ, van Os J, Leentjens AFG (2016) Unraveling the Relationship between Motor Symptoms, Affective States and Contextual Factors in Parkinson's Disease: A Feasibility Study of the Experience Sampling Method. PLoS ONE 11(3): e0151195. 
As long as we feel safely held in the hearts and minds of the people who love us, we will climb mountains and cross deserts and stay up all night to finish projects.

\author{
Bessel van der Kolk
}




\section{DANKWOORD}

Lieve Richel, dat onze wegen zich hebben mogen kruizen, is misschien wel één van de meest dierbare 'bijproducten' van mijn hele promotie-avontuur. Dat mijn proefschrift überhaupt tot stand is gekomen, ondanks alle uitdagingen, tegenslagen en worstelingen onderweg, dank ik op de eerste plaats aan jou. Aan je enorme kennis en kunde, je eeuwige geduld als ik weer eens een syntax-blunder had begaan, of niet meer wist welke versie dataset nu ook alweer de juiste was. Aan je luisterende oor, je vertrouwen in mij, en dat je het steeds opnieuw weer uitsprak op de momenten dat ik het in mezelf verloor. Aan alle waardevolle gesprekken die we voerden, die soms over een AR-1 structuur of 3e-orde interactieterm gingen, maar vaker vooral ook niet. Aan het feit dat je er al die jaren voor me bent geweest en letterlijk en figuurlijk je deur voor me open zette. Dank ook aan je lieve gezin, die me net zo hartelijk welkom hebben geheten, en meer dan eens van koffie, thee en koekjes hebben voorzien. De afgelopen jaren waren one hell of a ride en wat ben ik dankbaar dat je ondanks al de omwegen en onvoorziene obstakels, tot aan de finish naast me bent blijven staan.

I $\mathrm{k}$ had het zonder jou niet gered.

Beste Jim, lieve Jim, wat kan ik zeggen... je bent een uniek exemplaar. Ik weet nog dat ik in mijn tweede studiejaar een debat over schizofrenie moest voeren, in het 'het is geen hersenziekte-kamp' werd ingedeeld, en me al inlezend in tegenargumenten op artikelen van een zekere van Os stuitte. Een beste man, vond ik toen al. Een jaar later las ik je boek over persoonlijke diagnostiek en vond dat een verademing. Toch nog iemand die het wél leek te begrijpen, dacht ik al lezend met regelmaat. Kort daarna trok ik mijn stoute schoenen aan en vroeg je in verband met een persoonlijke kwestie om hulp. Jouw reactie, die ik eerlijk gezegd niet eens verwachtte, volgde dezelfde zondagmiddag nog. We dronken koffie, spraken over de GGZ en wat er allemaal aan moest veranderen, en voor ik het wist had ik als Hollander in een Limburgs ziekenhuis en kamer op de poli psychiatrie om het nieuwe ROMmen uit te werken, belde ik met willekeurige Nederlanders over hun al dan niet psychotische belevingen en stond ik hersenscans te maken in een van de meeste geavanceerde MRI-scanners ter wereld. Met het leven weet je het uiteindelijk allemaal nooit. Zo belandde ik even toevallig en plots in de hele Schizofrenie Bestaat Niet campagne, met een 6-jaar durend redacteurschap van PsychoseNet tot gevolg. En oh ja, ook nog in een promotietraject. Dat zou wel goedkomen, zei je.

En het kwam goed, al bewandelde ik, en bewandelden wij, bepaald geen gebaande wegen en liep ongeveer alles anders dan 'normaal'. Dat was niet per se makkelijk, maar het gaf me wel de vrijheid en ruimte om mijn eigen passie en interesses te volgen, ontzettend veel te leren, soms te vallen, steeds weer op te staan. Ik ben je dan 
ook immens dankbaar voor alle kansen die je me hebt geboden en de wereld die er voor me is opengegaan sinds ons eerste contact. Ik heb grote bewondering voor wie je bent, met hoeveel energie je jouw leven wijdt aan de wereld een beetje mooier en makkelijker maken (al zullen sommigen daar misschien ook wel anders over denken...), je humor, je stelligheid, je toegankelijkheid, voor alle talen die je spreekt, alle dingen die je weet, maar zeker ook je besef van alles wat we vooral niet weten. You are a true force to be reckoned with, en wat was het me een waar genoegen. Graag tot het volgende avontuur!

Lieve Katinka, als iemand me door diepe dalen heen heeft gesleurd, en steeds op mijn terugkomst heeft gewacht, ben jij het. Dankbaar dat je altijd bent gebleven, dat je me ongeacht mijn status en capuchontrui gewoon laat zijn, intens dankbaar dat je er bent.

Lieve Sjerty, Wim, Gorry, Linda, Renate, Ferdinand, gedurende verschillende perioden in mijn leven hebben jullie me ondersteund, beluisterd, geholpen en me elk op eigen wijze handvatten aangereikt en stapjes vooruitgeholpen. Jullie kennis en ervaring koester ik en draag ik met me mee, waarvoor mijn diepste dank.

Lieve Maggie, in een jaar dat haast in zijn geheel een dieptepunt vormde, was het knutselen met jou een hoogtepunt dat ik, het verdere arrangement en de toelatingseisen daargelaten, voor geen goud had willen missen. Zelden heb ik ervaren hoe troostend het kan zijn om je zo gekend, erkend en herkend te voelen, ondanks, maar misschien ook wel dankzij, gebruik van relatief weinig woorden. Dank voor al onze telefoongesprekken in het afgelopen jaar, je zwarte en soms hele foute humor - en zeker ook het kunnen waarderen van die van mij -, je kennis, je luisteren, je delen en de wetenschap dat ik op momenten waarin ik mij zo alleen en (ver)vreemd voel, weet dat ik dat uiteindelijk nooit ben. Je bent een ontzettend leuk mens, en ik vind het een voorrecht je te kennen.

Lieve Wilma, in al mijn geworstel rondom het schrijven van dit proefschrift was jij vaak in gedachten bij me, en voelde je als mijn bondgenoot. Dank voor al jouw belangrijke werk en de weg die je voor mij hebt vrijgemaakt om verder te bewandelen.

Lieve Irene en Klazine, hoewel onze wegen zich in de afgelopen jaren fysiek nauwelijks hebben gekruist, voel ik me met jullie verbonden. Dank voor al jullie harde, goede en inspirerende werk. Voor de weg die jullie elk afzonderlijk, maar zeker ook gezamenlijk, hebben afgelegd, en hoe jullie nu anderen helpen op die van hen. Ik ben oprecht heel trots op jullie, en hoop dat onze wegen nog vaker zullen kruizen. 
Lieve Annemarije, fijn mens, ook met jou voel ik me verbonden. Dank voor je steun in de afgelopen maanden, onze mooie gesprekken, alle goede dingen die je doet. Ik kijk er enorm naar uit op een dag met je samen te werken en onze gezamenlijke kennis, kunde en ervaring te kunnen te bundelen tot iets heel moois.

Lieve Linda, kamergenote in Corona-tijden, mogelijk besef je je niet hoe groot je aandeel is geweest in het tot stand komen van dit proefschrift, dus bij deze: je aandeel erin was indirect doch groot. Dank voor je fijne energie, dat ik alle muren vol mocht hangen met planningen en lijstjes, voor de koffie, de noodles, je humor en dat ik ongeacht kledingstijl (meestal dezelfde) en stemming (stijgende lijn) gewoon mocht zijn.

Lieve Nienke, hoe anders zou mijn en vooral ook ons leven eruitzien zonder jou. Ik bewonder je energie, je flexibiliteit, je grote hart voor de mensheid en zeker ook je passie om mensen te laten meedoen, en winnen. Dank voor wie je bent, en dat je er bent!

Lieve Dorota, ik denk dat je half niet weet hoe bijzonder je bent. Bedankt voor al je steun, je rust, je energie en je krachtige maar bovenal liefdevolle aanraking van mijn lijf.

Beste Philippe, Jacqueline, Wiepke, Esther en Wilma, leden van mijn promotiecommissie, lieve mensen, dank voor het deel uitmaken van de afronding van mijn promotie-avontuur en de tijd die jullie daarvoor vrij hebben willen maken. Kers op de spreekwoordelijke taart!

Lieve mensen van Vijverdal en omstreken, in ieder geval Truda, Trees, Ron, Karel, Marga, Flore, Maarten, Elaine, Suzanne, Simone, Carine, en Rosan, fijn dat jullie links- of rechtsom onderdeel hebben uitgemaakt van mijn Vijverdal-tijd. Hoewel ik er in de regel eerder niet dan wel was te vinden, hebben onze paden zich op verschillende manieren toch gekruist. Dank voor de (praktische) hulp, ondersteuning en/of interessante en gezellige gesprekken.

Lieve Netty, bij UM SPORT heb ik me jaren thuis gevoeld, waarvoor ik vooral jou wil bedanken. Het heeft, in alle gekte die er soms heerste, veel voor me betekend ergens zo welkom te zijn. Genoten van onze samenwerking, maar vooral ook gewoon van jou. 
Dear Elisabeth, Coolest, we go way back to a time where I walked around with a stuffed animal, and you were Head of Kitchen. You made me feel so welcome and seen during a time in my life where I felt so lost. It meant the world to me. We've shared so many beautiful moments, walks and talks since then, and now even reached the point of discussing my dissertation together. Which, since you are probably the most intelligent woman I've ever met, felt like a true privilege to me. Heartfelt thank you, for you.

Lieve Mayla en Flynn, dank voor alle (onbewuste) lessen die jullie me leren, en dat ik ondanks mijn chronische gezeur om schoenen en groenten in jullie leven mag zijn. Met liefs, en een dikke vette telemark landing :-)

Lieve papa en mama, zonder jullie was er bij voorbaat niets van dit al geweest, om meer dan alleen een praktische reden. Ondanks de misschien her en der wat onfortuinlijke genen en dito geërfde karaktereigenschappen, ben ik jullie eindeloos dankbaar voor jullie nimmer aflatende trots op/om/voor mij en alle liefde en vertrouwen die ik van jullie heb meegekregen om te kunnen en mogen doen wat ik allemaal heb gedaan. Hoewel we vrees ik nooit echt van zelfvertrouwen zullen blaken, durf ik zeker ook dankzij jullie steun en liefde zelf en met vertrouwen in de wereld te staan.

Lieve Frank, mijn allerliefste Frank. Ik denk dat je gelijk hebt als je wel eens zegt dat niemand ooit zoveel van me gehouden heeft, als jij. Ik denk ook dat ik gelijk heb, als ik je zeg dat niemand ooit zoveel van jou gehouden heeft, als ik. Ik wist niet dat het kon, en ik wist niet dat het bestond. Ik ben je dankbaar voor zo ontzettend veel dingen, maar alles samengevat, het meest vooral voor jou. Voor wie je bent, voor dat je er bent en voor hoe donker het in mij soms ook kan worden, ik altijd en onvoorwaardelijk op jouw licht en liefde kan rekenen. Je bent werelds en mijn wereld, waarvoor mijn allergrootste dank.

Tenslotte Maastricht, mooie, fijne stad. De plek waar ik me in eigen land jarenlang chronisch op vakantie voelde, waar ik mezelf vond, maar ook vreselijk verloor. Waar ik getergd door eindeloze insomnia meer dan eens door nachten zwierf, maar ook altijd weer thuiskwam. In collegebanken zat, koffiedronk, veel koffiedronk, rondjes rende, rondjes fietste, bijzondere mensen ontmoette, diepte- maar minstens zoveel hoogtepunten beleefde. Lieve Maastricht, het was me allemaal wat, niet op de laatste plaats een groot genoegen. 


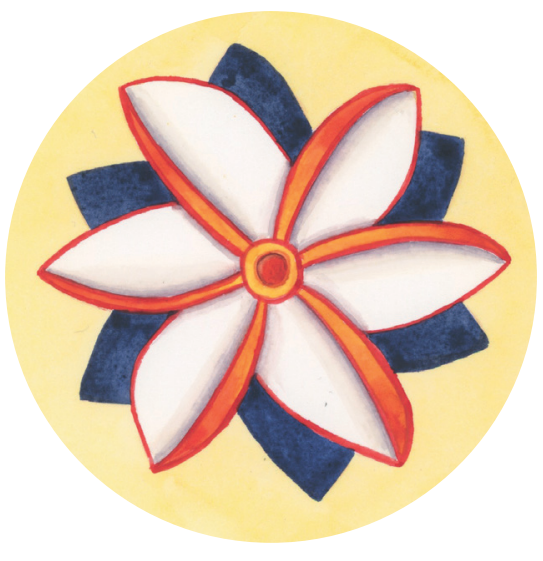

"It's about shame, disgust, and losing every sense of dignity. About darkness, silence and secrecy. It's about sadness, loneliness, anger and fear. About not being able to express myself or how I feel, and having no boundaries or protection. Basically, it's about not knowing how to just sit, feel and breathe through whatever difficult thought or emotion - without moving to hide it, or fade it, or fix it. It's about looking for safety and comfort in all the wrong directions, and relying on self-destruction in order to live and survive."

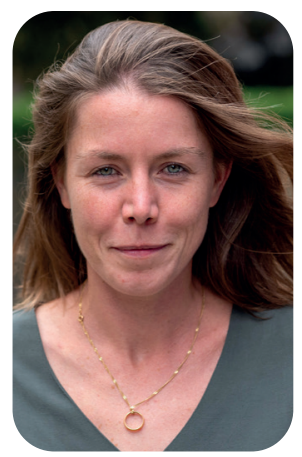

Anne Marsman (1989) studied at Maastricht University, where she obtained a Bachelor's degree in Health Sciences, an Honours degree in International Health, and a Master's degree in Mental Health. In 2016, she started her PhD trajectory at the School for Mental Health and Neuroscience, in which she investigated the long-lasting impact of childhood adversity. In her dissertation, Anne combined scientific knowledge with her lived experience, as well as her professional experience as a psychologist. 
